\title{
WIDE COOL AND ULTRACOOL COMPANIONS TO NEARBY STARS FROM Pan-STARRS 1
}

\author{
Niall R. Deacon ${ }^{1,2}$, Michael C. Liu'2,10, Eugene A. Magnier ${ }^{2}$, Kimberly M. Aller ${ }^{2}$, William M. J. Best ${ }^{2}$, \\ Trent DupuY ${ }^{3,11}$, Brendan P. Bowler ${ }^{2,4,12,13}$, Andrew W. ManN ${ }^{5}$, Joshua A. Redstone ${ }^{6}$, William S. Burgett ${ }^{2}$, \\ Kenneth C. Chambers ${ }^{2}$, Peter W. Draper ${ }^{7}$, H. Flewelling ${ }^{2}$, Klaus W. Hodapr ${ }^{8}$, Nick Kaiser ${ }^{2}$, Rolf-Peter Kudritzki ${ }^{2}$, \\ Jeff S. Morgan ${ }^{2}$, Nigel Metcalfe ${ }^{7}$, Paul A. Price ${ }^{9}$, John L. Tonry ${ }^{2}$, and Richard J. Wainscoat ${ }^{2}$ \\ ${ }^{1}$ Max Planck Institute for Astronomy, Koenigstuhl 17, D-69117 Heidelberg, Germany; deacon@ mpia. de \\ ${ }^{2}$ Institute for Astronomy, University of Hawai ${ }^{1}$ i, 2680 Woodlawn Drive, Honolulu, HI 96822, USA \\ ${ }^{3}$ Harvard-Smithsonian Center for Astrophysics, 60 Garden Street, Cambridge, MA 02138, USA \\ ${ }^{4}$ California Institute of Technology, Division of Geological and Planetary Sciences, 1200 East California Boulevard, Pasadena, CA 91125, USA \\ ${ }^{5}$ Harlan J. Smith Fellow, Department of Astronomy, The University of Texas at Austin, Austin, TX 78712, USA \\ ${ }^{6}$ Equatine Labs, 89 Antrim Street, \#2, Cambridge, MA 02139, USA \\ ${ }^{7}$ Department of Physics, University of Durham, South Road, Durham DH1 3LE, UK \\ ${ }^{8}$ Institute for Astronomy, University of Hawai 'i, 640 North Aohoku Place, Hilo, HI 96720, USA \\ ${ }_{9}^{9}$ Princeton University Observatory, 4 Ivy Lane, Peyton Hall, Princeton University, Princeton, NJ 08544, USA \\ Received 2013 November 8; accepted 2014 July 8; published 2014 August 22
}

\begin{abstract}
We present the discovery of 57 wide $\left(>5^{\prime \prime}\right)$ separation, low-mass (stellar and substellar) companions to stars in the solar neighborhood identified from Pan-STARRS 1 (PS1) data and the spectral classification of 31 previously known companions. Our companions represent a selective subsample of promising candidates and span a range in spectral type of K7-L9 with the addition of one DA white dwarf. These were identified primarily from a dedicated common proper motion search around nearby stars, along with a few as serendipitous discoveries from our Pan-STARRS 1 brown dwarf search. Our discoveries include 23 new L dwarf companions and one known L dwarf not previously identified as a companion. The primary stars around which we searched for companions come from a list of bright stars with well-measured parallaxes and large proper motions from the Hipparcos catalog (8583 stars, mostly A-K dwarfs) and fainter stars from other proper motion catalogs (79170 stars, mostly M dwarfs). We examine the likelihood that our companions are chance alignments between unrelated stars and conclude that this is unlikely for the majority of the objects that we have followed-up spectroscopically. We also examine the entire population of ultracool ( $>$ M7) dwarf companions and conclude that while some are loosely bound, most are unlikely to be disrupted over the course of $\sim 10$ Gyr. Our search increases the number of ultracool M dwarf companions wider than $300 \mathrm{AU}$ by $88 \%$ and increases the number of $\mathrm{L}$ dwarf companions in the same separation range by $82 \%$. Finally, we resolve our new L dwarf companion to HIP 6407 into a tight (0' $13,7.4 \mathrm{AU}) \mathrm{L} 1+\mathrm{T} 3$ binary, making the system a hierarchical triple. Our search for these key benchmarks against which brown dwarf and exoplanet atmosphere models are tested has yielded the largest number of discoveries to date.
\end{abstract}

Key words: binaries: general - brown dwarfs - stars: low-mass - surveys

Online-only material: color figures

\section{INTRODUCTION}

Wide ( $\gtrsim 100 \mathrm{AU}$ ) binary companions have long been used as a tool for identifying and studying faint stellar and substellar objects. Such systems are relatively common, with at least $4.4 \%$ of solar-type stars having a companion wider than $2000 \mathrm{AU}$ (Tokovinin \& Lépine 2012) and $\sim 25 \%$ having companions wider than $100 \mathrm{AU}$ (Raghavan et al. 2010). Indeed, the Sun's closest stellar neighbor, Proxima Centauri, is a $\sim 15,000 \mathrm{AU}$ common proper motion companion to Alpha Centauri (Innes 1915). These objects are an important population for understanding models of binary star formation. The widest systems may have been formed by capture within a young cluster

\footnotetext{
${ }^{10}$ Visiting Astronomer at the Infrared Telescope Facility, which is operated by the University of Hawaii under Cooperative Agreement No. NNX-08AE38A with the National Aeronautics and Space Administration, Science Mission Directorate, Planetary Astronomy Program.

${ }^{11}$ Hubble Fellow.

12 Visiting Astronomer, Kitt Peak National Observatory, National Optical Astronomy Observatory, which is operated by the Association of Universities for Research in Astronomy (AURA) under cooperative agreement with the National Science Foundation.

${ }^{13}$ Caltech Joint Center for Planetary Astronomy Fellow.
}

(Kouwenhoven et al. 2010), a mechanism that has been used to explain the apparent increase in the number of companions per log separation bin at separations over 20,000 AU (Dhital et al. 2010). Another possibility is that these objects formed closer in and were pushed out to wider orbits by three-body interactions (Delgado-Donate et al. 2004; Umbreit et al. 2005; Reipurth \& Mikkola 2012). In this scenario the wide companion fraction should be higher for close binary systems. Indeed Law et al. (2010) have found that $45_{-16}^{+18} \%$ of wide $\mathrm{M}$ dwarf systems were resolved as hierarchical triples with high-resolution imaging.

Wide binaries also provide test cases for characterizing stellar and substellar properties. As these systems likely formed from the same birth cluster, the companions will have the same metallicity and age as their host stars. Hence if one component has these parameters determined, the values can be applied to the other component. For example, wide $\mathrm{M}$ dwarf companions to FGK stars have been used as calibrators for spectroscopic determinations of $\mathrm{M}$ dwarf metallicity relations (Rojas-Ayala et al. 2010; Mann et al. 2013a, 2014). This "benchmarking" process is even more important for substellar objects as brown dwarfs lack a stable internal energy source and hence exhibit a degeneracy between their mass, luminosity, and age. 
For substellar companions this degeneracy can be broken using the age of the primary (in combination with the bolometric luminosity derived from their absolute magnitude and spectrum of the secondary) to estimate the radius, mass, and effective temperature of the secondary from evolutionary models. This effective temperature can then be compared to that derived from model fits to the secondary's spectrum, testing the agreement between atmospheric and evolutionary models (e.g., Saumon et al. 2007; Deacon et al. 2012b). There are also a handful of systems where the ultracool ${ }^{14}$ secondary itself is a binary. Such very rare systems are not simply "age benchmarks" (Liu et al. 2008); since the mass of the secondary is measured dynamically, they provide the opportunity for even more stringent tests of theoretical models (Dupuy et al. 2009).

As a result of their importance, wide substellar companions have been an active area of study in recent years. Many substellar companions have been identified as byproducts of larger searches for brown dwarfs (e.g., Burningham et al. 2009) or by matching known brown dwarfs with catalogs of known stars (e.g., Faherty et al. 2010; Dupuy \& Liu 2012). Dedicated large-scale companion searches such as Pinfield et al. (2006) are more rare. A summary of discoveries prior to 2010 is presented in Faherty et al. (2010). Since then wide-field surveys such as Sloan Digital Sky Survey (SDSS; Ahn et al. 2012; see studies by Zhang et al. 2010; Dhital et al. 2010) and WISE (Wright et al. 2010; see work by Luhman et al. 2012; Wright et al. 2013) have been used to identify wide companions to stars. As these surveys are either single epoch or taken over a short period of time, they often require additional data sets, such as Two Micron All Sky Survey (2MASS; Skrutskie et al. 2006), to allow the identification of companions from their common proper motion with the primary. Hence the ideal tool for identifying wide, lowmass companions to stars is a red-sensitive, wide-field, multiepoch survey.

Pan-STARRS 1 is a wide-field $1.8 \mathrm{~m}$ telescope situated on Haleakala on Maui in the Hawaiian Islands. Run by a consortium of astronomical research institutions, it has been surveying the sky north of $\delta=-30^{\circ}$ since 2010 May. Just over half of the telescope's operating time is reserved for the $3 \pi$ Survey, a multi-filter, multi-epoch survey of three-fourths of the sky $\left(\sim 30,000 \mathrm{deg}^{2}\right)$. The Pan-STARRS 1 photometric system is defined in Tonry et al. (2012) and consists of five filters used for the $3 \pi$ Survey $\left(g_{P 1}, r_{P 1}, i_{P 1}, z_{P 1}\right.$, and $\left.y_{P 1}\right)$ as well as an extrawide $w_{P 1}$ filter specially designed for asteroid searches. It is the $y_{P 1}$ filter, centered on 0.99 microns $(\delta \lambda=70 \mathrm{~nm})$, which makes Pan-STARRS 1 ideal for surveying the local population of brown dwarfs. So far over $100 \mathrm{~T}$ dwarfs have been identified (Deacon et al. 2011; Liu et al. 2011; M. C. Liu et al., in preparation), many in the early-T regime (Best et al. 2013). Such objects were often missed by previous surveys due to their indistinct colors in the near-infrared compared to background $\mathrm{M}$ dwarfs. However, the addition of Pan-STARRS 1 astrometry and farred optical photometry disentangles them from the much larger number of $\mathrm{M}$ dwarfs with similar near-infrared colors.

As a wide-field multi-epoch survey, Pan-STARRS 1 also provides an ideal data set for identifying wide, common proper motion companions to nearby stars. This is a natural extension of our search for nearby field brown dwarfs and involves searching the Pan-STARRS 1 proper motion database for objects moving with a common proper motion to known stars. This approach

\footnotetext{
14 A term typically used to mean objects of spectral type M7 or later. These may be free-floating planetary mass objects, brown dwarfs, or very low-mass stars, depending on the spectral type and age of the object.
}

Table 1

Summary Information on the Sources for Our Input Primary List

\begin{tabular}{lccc}
\hline \hline Survey & Reference & $n_{\text {total }} n_{\text {passed }}{ }^{\text {a }}$ & \\
\hline $\begin{array}{l}\text { Hipparcos } \\
\text { Lepine \& Gaidos }\end{array} \quad$ Lan Leeuwen (2007) & 117955 & 9466 \\
$\quad$ bright M dwarfs & & 8889 & 5432 \\
LSPM North & Lépine \& Shara (2005) & 61977 & 60793 \\
rNLTT & Salim \& Gould (2003) & 36085 & 31696 \\
Total $^{\text {b }}$ & & & 79593 \\
\hline
\end{tabular}

Notes.

a The number of objects that passed our Galactic latitude, total proper motion, and declination cuts and (for Hipparcos stars) our parallax.

b Total number excluding duplicate appearances of the same object in different catalogs.

has already led to the discovery of a wide T4.5 companion to the nearby K dwarf HIP 38939 (Deacon et al. 2012b). Additionally, objects discovered in our field brown dwarf search can be crossmatched with catalogs of nearby stars to serendipitously identify wide multiple systems. In Deacon et al. (2012a), we used this method to identify a wide T5 companion to the M dwarf LHS 2803 (simultaneously found by Mužić et al. 2012). We present here the results of our full search for wide cool and ultracool companions identified using PS1 proper motions.

\section{IDENTIFICATION IN PAN-STARRS1 DATA}

\subsection{Primary Star Selection}

To identify objects with proper motion in common with nearby stars, we first began by collating lists of stars to search in. We started with stars from the Hipparcos catalog (van Leeuwen 2007). To limit the contamination by distant background stars in our sample, we included only Hipparcos stars with proper motions above $00^{\prime} 1 \mathrm{yr}^{-1}$ and parallaxes more significant than $5 \sigma(d \lesssim 200 \mathrm{pc})$. This provided us with a relatively complete sample of stars in the solar neighborhood with spectral types A-K. We supplemented this catalog with lower-mass primaries drawn from the LSPM (Lépine \& Shara 2005; $\mu>0$ ". $15 \mathrm{yr}^{-1}$, $\delta>0^{\circ}$ ) and rNLTT (Salim \& Gould 2003; $\mu>0^{\prime \prime} .2 \mathrm{yr}^{-1}$ ) proper motion catalogs and with the bright $\mathrm{M}$ dwarf catalog of Lépine $\&$ Gaidos (2011) to which we applied a proper motion cut of $\mu>00^{\prime} .1 \mathrm{yr}^{-1}$. See Table 1 for details on the number of primary stars from each input catalog.

\subsection{Selection of Companions from Pan-STARRS 1 Data}

Pan-STARRS 1 is an ongoing survey and as such the available data products are constantly evolving. We conducted our search over several iterations of the Pan-STARRS $13 \pi$ database. In all our searches we queried the most up-to-date Pan-STARRS 1 $3 \pi$ database using the Desktop Virtual Observatory software (Magnier et al. 2008). Initially, our search involved combining 2MASS and Pan-STARRS 1 data to calculate proper motions in a similar process to that used by Deacon et al. (2011). For this PS1 + 2MASS search, we required that objects had more than one detection in the $y_{P 1}$ band. PS1 detections were required to have a significance greater than $5 \sigma$ to be included in the astrometric and photometric solutions of their parent object. We also required that the objects be classified as good quality, point-source detections in both Pan-STARRS 1 and 2MASS.

Since 2012 June, we have used proper motions calculated using Pan-STARRS 1 only data (although 2MASS data is included if a detection is within $1^{\prime \prime}$ of the mean Pan-STARRS 1 
position). In these cases, we required that the proper motion measurement of the candidate companion was more significant than $5 \sigma$, be calculated from more than seven position measurements, and that the time baseline over which it was calculated be greater than 400 days. Note that the Pan-STARRS 1 survey strategy consists of pairs of observations in the same filter taken $\sim 25$ minutes apart and often two filters will be taken in the same night. Hence our requirement of seven position measurements does not imply seven independent epochs evenly spread across the time baseline of the proper motion calculation.

In the case of the Hipparcos and Lépine \& Gaidos (2011) catalogs, distance estimates were available for all our target stars. Hence we searched for companions out to projected separations of 10,000 AU. For the LSPM and rNLTT catalogs, where distance estimates are not available for all the stars, we used a maximum search radius of 20 arcmin. In all cases we made an initial cut on our candidates, requiring that candidates have proper motions that agree with their supposed primaries' proper motions to within $0^{\prime} .1 \mathrm{yr}^{-1}$ in both R.A. and declination. This initial cut uses a tolerance that is much larger than our typical Pan-STARRS 1 proper motion errors of $\sim 5 \mathrm{mas} \mathrm{yr}^{-1}$. Next, we made much more stringent cuts on proper motion to select only likely companions.

We restricted our search to objects which had proper motion differences compared to their primaries that were less significant than $5 \sigma$; here $\sigma$ is the quadrature sum of the proper motion difference in each axis divided by the total proper motion error in that axis.

$$
\sigma^{2}=\frac{\left(\mu_{\alpha, 1}-\mu_{\alpha, 2}\right)^{2}}{\sigma_{\mu_{\alpha, 1}}^{2}+\sigma_{\mu_{\alpha, 2}}^{2}}+\frac{\left(\mu_{\delta, 1}-\mu_{\delta, 2}\right)^{2}}{\sigma_{\mu_{\delta, 1}}^{2}+\sigma_{\mu_{\delta, 2}}^{2}}
$$

Our typical proper motion errors in Pan-STARRS $1+2$ MASS

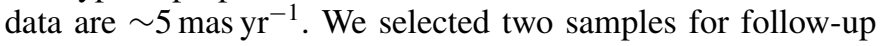
observations. In all cases we set a zone of exclusion around the Galactic center $|b|<5^{\circ}$ and $l<90$ or $l>270$.

1. Ultracool companions. Objects with red Pan-STARRS1 colors $\left(z_{P 1}-y_{P 1}>0.8 \mathrm{mag}\right)$ or $\left(y_{P 1}-J_{2 \mathrm{MASS}}>1.8 \mathrm{mag}\right)$ were selected as candidate ultracool companions. These cuts should only select objects of spectral types M7 or later (Deacon et al. 2011). These targets also had their Pan-STARRS 1 and 2MASS colors inspected to ensure they truly were red objects and had not entered our sample due to erroneous $z_{P 1}$ or $y_{P 1}$ photometry. This involved removing objects with more than one $g_{P 1}$ detection or with blue $i_{P 1}-y_{P 1}$ colors.

Note that two objects (the companions to LSPM J2153+1157 and NLTT 39312) are both close companions that did not meet our $z_{P 1}-y_{P 1}$ color cut but were selected due to their red $y_{P 1}-J_{2 \text { MASS }}$ colors $(>1.6)$ and close proximity $\left(<12^{\prime \prime}\right)$ to their primaries. These were subsequently spectrally typed as ultracool dwarfs. These two objects may have had their photometry affected by the proximity of their primary.

This sample yielded a total of 38 new discoveries, one of which (HIP 13589B) was simultaneously discovered by Allen et al. (2012). We have also re-identified and typed the NLTT 22073 system originally identified by Deacon \& Hambly (2007) and HIP 78184 B originally proposed as a candidate companion by Pinfield et al. (2006).

2. Bright companions. We selected the brightest of our common proper motion candidates $\left(J_{2 \mathrm{MASS}}<15.5 \mathrm{mag}\right.$ ) for a poor-weather back-up program for our NASA IRTF SpeX observations regardless of their color. These objects were mostly within an arcminute of the primary, with all having separations smaller than $3^{\prime}$. This resulted in the discovery of 16 new, bright companions and the typing of 26 previously known companions.

\subsection{Serendipitous Discoveries}

As part of our ongoing search for T dwarfs in Pan-STARRS 1 data (see Deacon et al. 2011 and Liu et al. 2011 for more details), we have been obtaining follow-up near-infrared photometry of Pan-STARRS 1 selected candidates. UKIDSS (Lawrence et al. 2007) or VISTA (Emerson \& Sutherland 2010) data were used where available; otherwise our candidate $\mathrm{T}$ dwarfs were observed using WFCAM (Casali et al. 2007) on the United Kingdom Infrared Telescope (UKIRT). We then crossmatched all our observed candidates with our combined proper motion catalog from Lépine \& Shara (2005), Salim \& Gould (2003), and Lépine \& Gaidos (2011) with a pairing radius of $20^{\prime}$. Objects that had $y_{P 1}-J_{2 \text { MASS }}>2.2$ mag were initially selected to be $\mathrm{T}$ dwarf candidates. However, if the additional near-IR photometry did not match our $y_{P 1}-J_{\mathrm{MKO}}>2.4 \mathrm{mag}$ and $J_{\mathrm{MKO}}-H_{\mathrm{MKO}}<0.7 \mathrm{mag}$ high priority $\mathrm{T}$ dwarf cut, but still met our $y_{P 1}-J_{\mathrm{MKO}}>2.0 \mathrm{mag}$ criterion, those objects were considered to be candidate L dwarfs. Any of these $\sim 500$ candidates that then had proper motions which did not deviate by more than $5 \sigma$ from their supposed primary stars were selected as candidate L companions for this search.

One of the serendipitous candidates we selected was a possible companion to the late-type high proper motion star NLTT 730. After searching the SIMBAD database, we identified our candidate object as 2MASS J00150206+2959323, a blue L7.5 dwarf found by Kirkpatrick et al. (2010) as a field object. As a blue L benchmark, this is similar to the G 203-50AB system identified by Radigan et al. (2008) although it is substantially wider (5070 AU versus $135 \mathrm{AU}$ ). This object is included in our subsequent analysis. In total we selected five serendipitous candidate companions.

Our proper motion companions are reported in Tables 2 and 3. A comparison between their proper motions and photometric distances (see Section 4.1.1) and the proper motions and distances of their primaries are shown in Table 4 for the Hipparcos companions and Table 5 for the companions to $\mathrm{M}$ dwarfs. In all our tables, the proper motions from the secondaries come from Pan-STARRS $1+2 \mathrm{MASS}$ proper motions. Where the object's 2MASS position was not included in the initial astrometric analysis we recalculated the proper motion solution including the 2MASS data. The proper motions and distances for all of our Hipparcos primaries come from van Leeuwen (2007).

\section{FOLLOW-UP OBSERVATIONS AND ARCHIVAL DATA}

\subsection{Infrared Photometry}

Where companions have confused or noisy photometry, we have used UKIRT/WFCAM (Casali et al. 2007) to acquire additional near-infrared photometry. These data were reduced at the Cambridge Astronomical Survey Unit using the WFCAM survey pipeline (Irwin et al. 2004; Hodgkin et al. 2009). See Table 6 for the near-infrared photometry of our companions from our UKIRT observations, 2MASS (Skrutskie et al. 2006), UKIDSS (Lawrence et al. 2007), VISTA; (Emerson \& Sutherland 2010), pre-release photometry from the UKIRT Hemisphere Survey (UHS; S. Dye et al., in preparation, accessed through the 
Table 2

Summary Information on Our Wide Companions

\begin{tabular}{|c|c|c|c|c|c|c|c|c|c|c|c|}
\hline Object & $\begin{array}{c}\text { WDS } \\
\text { Designation }\end{array}$ & $\begin{array}{c}\text { Position } \\
(\text { Eq. }=\mathrm{J} 2000 \text { Ep. }=2010.0)\end{array}$ & $\begin{array}{l}\text { SpT } \\
\text { Primary }\end{array}$ & $\begin{array}{c}\text { SpT } \\
\text { Secondary }\end{array}$ & $\begin{array}{c}y_{P 1} \\
(\mathrm{mag})\end{array}$ & $\begin{array}{l}J_{2 \mathrm{MASS}} \\
(\mathrm{mag})\end{array}$ & $\begin{array}{l}d_{\text {primary }} \\
(\mathrm{pc})\end{array}$ & $\begin{array}{l}\text { Separation } \\
\left({ }^{\prime \prime}\right)\end{array}$ & $\begin{array}{l}\text { Separation } \\
\text { (AU) }\end{array}$ & $\begin{array}{l}\text { Metallicityk } \\
\quad(\text { dex })\end{array}$ & $\begin{array}{l}\text { Age } \\
(\mathrm{Gyr})\end{array}$ \\
\hline \multicolumn{12}{|c|}{ HIP search companions } \\
\hline HIP 2397 B & $\ldots$ & $003024.94+224447.1$ & K5 & L0.5 & $16.606 \pm 0.008$ & $14.586 \pm 0.038$ & {$\left[33.9_{-1.3}^{+1.4}\right]$} & 117.1 & 3970 & $\ldots$ & $0.5-\sim 10^{\mathrm{y}}$ \\
\hline HIP $6217 \mathrm{C}$ & $\ldots$ & $011944.74+000618.5$ & K0 & M9.5 & $16.859 \pm 0.009$ & $15.441 \pm 0.060$ & {$\left[70.9_{-11.7}^{+14.0}\right]$} & 27.4 & 2767 & $\ldots$ & $0.4-\sim 10^{\mathrm{y} 1}$ \\
\hline HIP 6407 B & $\ldots$ & $012216.99+033122.0$ & G5 & $\mathrm{L} 1+\mathrm{T}^{1}$ & $17.527 \pm 0.027$ & $15.471 \pm 0.046$ & {$\left[57.2_{-3.0}^{+3.3}\right]$} & 44.9 & 2570 & $\ldots$ & $0.5-\sim 10^{\mathrm{y}}$ \\
\hline HIP $9269 \mathrm{~B}^{\ddagger}$ & $\ldots$ & $015910.98+331227.8$ & G5 & L6 & $18.266 \pm 0.031$ & $15.956 \pm 0.088$ & {$\left[25.0_{-0.4}^{+0.3}\right]$} & 52.1 & 1300 & 0.15 & $2.2-10.2^{\mathrm{i}}$ \\
\hline HIP 10033 B & $\ldots$ & $020910.58-021919.0$ & F8 & M5 & $15.14 \pm 0.003$ & $13.761 \pm 0.027$ & {$\left[69.7_{-3.1}^{+3.4}\right]$} & 42.9 & 2990 & -0.08 & $2.7-3.7^{\mathrm{j}}$ \\
\hline HIP 11161 B & $\ldots$ & $022336.59+524005.9$ & F5 & L1.5 & $18.785 \pm 0.067$ & $16.636 \pm 0.161$ & {$\left[69.3_{-3.6}^{+4.1}\right]$} & 47.7 & 3300 & -0.23 & $0.5-2.4^{\mathrm{j}}$ \\
\hline HIP $13589 \mathrm{~B}^{*}$ & $\ldots$ & $025507.10+213621.1$ & G0 & M7.5 & $17.098 \pm 0.009$ & $14.928 \pm 0.034$ & {$\left[72.8_{-4.0}^{+4.6}\right]$} & 49.2 & 3580 & $\ldots$ & $0.4-\sim 10^{\mathrm{y}}$ \\
\hline \multirow{2}{*}{ HIP 26653 B } & $\ldots$ & $053949.51+525358.3$ & G5 & L5 & $16.905 \pm 0.012$ & $14.756 \pm 0.044$ & {$\left[18.8_{-3.1}^{+3.7}\right]$} & 27.0 & 753 & -0.07 & $1.1-9.3^{\mathrm{i}}$ \\
\hline & & & & & & & & & & -0.1 & $1.6-13.8^{\mathrm{j}}$ \\
\hline HIP 32728 B & $\ldots$ & $064930.59+362626.0$ & G0 & M6.5 & $16.060 \pm 0.006$ & $14.508 \pm 0.031$ & {$\left[46.6_{-2.6}^{+2.9}\right]$} & 94.3 & 4400 & $\ldots$ & $0.3-\sim 10^{\mathrm{y}}$ \\
\hline HIP $37283 \mathrm{~B}^{\dagger}$ & $07394+5032$ B & $073932.03+503107.3$ & F5 & M6 & $14.214 \pm 0.002$ & $12.797 \pm 0.027$ & {$\left[45.5_{-1.2}^{+1.2}\right]$} & 106.1 & 4830 & -0.1 & $0.4-2.1^{\mathrm{j}}$ \\
\hline HIP 46984 B & $\ldots$ & $093433.18+033407.0$ & F8 & M4 & $14.521 \pm 0.002$ & $13.285 \pm 0.027$ & {$\left[74.7_{-6.9}^{+8.6}\right]$} & 37.5 & 2800 & $\ldots$ & $0.4-\sim 10^{\mathrm{y}}$ \\
\hline HIP $49046 B^{\dagger}$ & $10004+2716 \mathrm{~B}$ & $100035.71+271706.7$ & M0 & M6.5 & $14.482 \pm 0.002$ & $13.022 \pm 0.022$ & {$\left[34.7_{-2.3}^{+2.6}\right]$} & 136.1 & 4720 & $\ldots$ & $0.3-\sim 10^{z}$ \\
\hline HIP 51877 B & $\ldots$ & $103554.72+365541.0$ & G5 & M9.5 & $16.935 \pm 0.008$ & $15.205 \pm 0.063$ & {$\left[57.4_{-9.6}^{+11.5}\right]$} & 17.6 & 954 & $0.22^{\mathrm{u}}$ & $2.4-7.5^{\mathrm{i}}$ \\
\hline HIP $52463 \mathrm{~B}^{\dagger}$ & $10435-1852$ B & $104326.65-185218.2$ & G4 & M5 & $15.043 \pm 0.003$ & $13.703 \pm 0.024$ & {$\left[74.2_{-4.5}^{+5.2}\right]$} & 43.5 & 3230 & 0.14 & $1.0-4.9^{\mathrm{j}}$ \\
\hline HIP $55666 \mathrm{~B}^{\ddagger}$ & $\ldots$ & $112423.43-055409.8$ & F5 & M5.5 & $14.533 \pm 0.037$ & $12.674 \pm 0.024$ & {$\left[49.8_{-1.5}^{+1.5}\right]$} & 37.2 & 1850 & 0.14 & $3.6-5.1^{\mathrm{j}}$ \\
\hline HIP $58918 \mathrm{~B}^{\dagger}$ & $12049+3437$ B & $120452.15+343723.2$ & K1 & M7 & $15.849 \pm 0.004$ & $14.471 \pm 0.03$ & {$\left[55.8_{-5.1}^{+6.2}\right]$} & 20.0 & 1110 & $\ldots$ & $0.1-\sim 10^{\mathrm{y}}$ \\
\hline HIP $59310 \mathrm{~B}^{\dagger}$ & $12101+1859 \mathrm{~B}$ & $121009.79+185807.9$ & K5 & M7 & $15.252 \pm 0.002$ & $13.691 \pm 0.027$ & {$\left[44.4_{-2.8}^{+3.2}\right]$} & 82.1 & 3650 & $\ldots$ & $0.2-\sim 10^{y}$ \\
\hline HIP 59933 B & $\ldots$ & $121736.41+142711.7$ & F8 & L1 & $18.111 \pm 0.032$ & $16.101 \pm 0.088$ & {$\left[57.0_{-2.4}^{+2.7}\right]$} & 38.1 & 2170 & 0.03 & $0.3-2.5^{\mathrm{j}}$ \\
\hline HIP $60501 \mathrm{~B}^{\dagger}$ & $12241+0357 \mathrm{~B}$ & $122409.68+035603.3$ & M0 & M5 & $12.876 \pm 0.022$ & $11.378 \pm 0.027$ & {$\left[56.6_{-6.0}^{+7.6}\right]$} & 37.1 & 2100 & $\ldots$ & $0.3-\sim 10^{\mathrm{z}}$ \\
\hline HIP $60987 \mathrm{~B}^{\dagger \mathrm{c}}$ & $12300-0601$ B & $123001.60-060123.7$ & $\mathrm{~F} 2$ & K7 & $\ldots$ & $\ldots$ & {$\left[61.9_{-2.3}^{+2.4}\right]$} & $\sim 5$ & $\sim 300$ & -0.12 & $1.3-2.4^{j}$ \\
\hline HIP $60987 \mathrm{C}^{\dagger}$ & $12300-0601 \mathrm{C}$ & $123000.68-060117.9$ & $\mathrm{~F} 2$ & M3 & $12.553 \pm 0.001$ & $11.402 \pm 0.028$ & {$\left[61.9_{-2.3}^{+2.4}\right]$} & 19.0 & 1170 & -0.12 & $2.0^{\mathrm{j}}$ \\
\hline HIP $63506 C^{f}$ & $13008+4213 \mathrm{C}$ & $130050.27+421447.6$ & M0 & $\mathrm{L} 1$ & $16.999 \pm 0.008$ & $15.15 \pm 0.042$ & {$\left[42.5_{-3.9}^{+4.7}\right]$} & 132.8 & 5640 & $\ldots$ & $0.3-\sim 10^{\mathrm{z}}$ \\
\hline HIP $65706 \mathrm{~B}^{\dagger}$ & $13284+3005 \mathrm{~B}$ & $132820.77+300317.0$ & K7 & M7 & $15.016 \pm 0.003$ & $13.315 \pm 0.022$ & {$\left[51.1_{-4.0}^{+4.8}\right]$} & 52.6 & 2690 & $\ldots$ & $0.3-\sim 10^{\mathrm{z}}$ \\
\hline HIP $65780 C^{f}$ & $13291+1128 \mathrm{C}$ & $132906.09+112752.2$ & K0 & M5 & $14.327 \pm 0.001$ & $13.580 \pm 0.002^{\mathrm{h}}$ & {$\left[58.0_{-3.3}^{+3.7}\right]$} & 16.0 & 928 & $\ldots$ & $0.5-\sim 10^{\mathrm{y}}$ \\
\hline HIP $70623 \mathrm{~B}^{\dagger}$ & 14268-0511 B & $142645.74-051020.9$ & K0 & M5.5 & $13.619 \pm 0.003$ & $12.191 \pm 0.026$ & {$\left[72.6_{-4.7}^{+5.5}\right]$} & 41.9 & 3040 & 0.46 & $3.3-6.0^{\mathrm{i}}$ \\
\hline HIP 75310 B & $\ldots$ & $152310.01-102858.4$ & G5 & M5 & $14.668 \pm 0.003$ & $13.291 \pm 0.032$ & {$\left[66.9_{-5.1}^{+6.0}\right]$} & 22.9 & 1530 & -0.22 & $6.3-9.3^{\mathrm{j}}$ \\
\hline HIP 76456 B & $\ldots$ & $153656.27+295931.1$ & F5 & M6.5 & $14.753 \pm 0.002$ & $13.300 \pm 0.031$ & {$\left[36.8_{-0.6}^{+0.6}\right]$} & 36.1 & 1330 & -0.25 & $0.4-2.2^{\mathrm{j}}$ \\
\hline HIP 76641 B & $\ldots$ & $153859.53+435217.2$ & G5 & M3.5 & $12.158 \pm 0.001$ & $10.817 \pm 0.018$ & {$\left[82.1_{-4.7}^{+5.3}\right]$} & 42.1 & 3460 & $\ldots$ & $0.4-\sim 10^{y}$ \\
\hline HIP $78184 \mathrm{~B}^{\mathrm{m}}$ & $15578+5916 \mathrm{~B}$ & $155755.32+591425.3$ & M0 & M9 & $16.219 \pm 0.024$ & $14.32 \pm 0.031$ & {$\left[31.4_{-1.3}^{+1.4}\right]$} & 121.8 & 3820 & $\ldots$ & $0.3-\sim 10^{z}$ \\
\hline HIP $78859 \mathrm{~B}^{\dagger}$ & $16059+3500 \mathrm{~B}$ & $160549.62+345953.9$ & G0 & M5 & $13.57 \pm 0.009$ & $12.258 \pm 0.021$ & {$\left[57.2_{-2.1}^{+2.3}\right]$} & 42.6 & 2440 & $\ldots$ & $0.7-\sim 10^{y}$ \\
\hline HIP 78916 B & $\ldots$ & $160632.22+225334.5$ & G0 & M8 & $16.694 \pm 0.008$ & $15.227 \pm 0.043$ & {$\left[92.9_{-9.2}^{+11.5}\right]$} & 35.5 & 3294 & $\ldots$ & $0.4-\sim 10^{\mathrm{y}}$ \\
\hline HIP 78923 B & $\ldots$ & $160638.65+340619.3$ & G5 & M8 & $15.565 \pm 0.004$ & $14.052 \pm 0.048$ & {$\left[46.1_{-1.7}^{+1.8}\right]$} & 15.5 & 714 & $-0.1^{\mathrm{j}}$ & $0.4-4.0^{\mathrm{j}}$ \\
\hline HIP $79180 \mathrm{~B}^{\dagger}$ & $16097+6550 \mathrm{~B}$ & $160942.06+654918.1$ & K7 & M5 & $15.315 \pm 0.003$ & $13.941 \pm 0.022$ & {$\left[74.7_{-3.4}^{+3.7}\right]$} & 29.0 & 2170 & $\ldots$ & $0.3-\sim 10^{\mathrm{z}}$ \\
\hline HIP $80258 B^{\dagger}$ & $16231+3650 \mathrm{~B}$ & $162309.09+365050.2$ & K3 & M4 & $14.487 \pm 0.004$ & $13.113 \pm 0.021$ & {$\left[63.7_{-7.5}^{+9.6}\right]$} & 54.9 & 3500 & $\ldots$ & $0.1-\sim 10^{\mathrm{y}}$ \\
\hline HIP 81910 B & $\ldots$ & $164349.50-264840.2$ & $\mathrm{G} 2$ & M6 & $13.852 \pm 0.025$ & $12.44 \pm 0.024$ & {$\left[46.4_{-0.8}^{+0.9}\right]$} & 26.7 & 1240 & 0.19 & $4.0-5.8^{\mathrm{j}}$ \\
\hline HIP 82233 B & $\ldots$ & $164803.98-155756.7$ & $\mathrm{G} 2$ & M5.5 & $13.198 \pm 0.009$ & $11.889 \pm 0.024$ & {$\left[42.1_{-1.2}^{+1.3}\right]$} & 30.4 & 1280 & -0.05 & $0.7-4.7^{\mathrm{j}}$ \\
\hline HIP $83651 \mathrm{~B}^{\dagger}$ & $17058+0458 \mathrm{~B}$ & $170549.00+045724.8$ & K5 & M3 & $12.529 \pm 0.052$ & $10.599 \pm 0.024$ & {$\left[50.9_{-4.2}^{+4.9}\right]$} & 28.9 & 1470 & $\ldots$ & $0.1-\sim 10^{\mathrm{y}}$ \\
\hline
\end{tabular}


Table 2

(Continued)

\begin{tabular}{|c|c|c|c|c|c|c|c|c|c|c|c|}
\hline Object & $\begin{array}{c}\text { WDS } \\
\text { Designation }\end{array}$ & $\begin{array}{c}\text { Position } \\
(\text { Eq. }=\mathrm{J} 2000 \text { Ep. }=2010.0)\end{array}$ & $\begin{array}{c}\text { SpT } \\
\text { Primary }\end{array}$ & $\begin{array}{c}\text { SpT } \\
\text { Secondary }\end{array}$ & $\begin{array}{c}y_{P 1} \\
(\mathrm{mag})\end{array}$ & $\begin{array}{l}J_{2 \mathrm{MASS}} \\
(\mathrm{mag})\end{array}$ & $\begin{array}{l}d_{\text {primary }} \\
(\mathrm{pc})\end{array}$ & $\begin{array}{l}\text { Separation } \\
\left({ }^{\prime \prime}\right)\end{array}$ & $\begin{array}{l}\text { Separation } \\
\text { (AU) }\end{array}$ & $\begin{array}{l}\text { Metallicityk } \\
\quad(\operatorname{dex})\end{array}$ & $\begin{array}{l}\text { Age } \\
(\mathrm{Gyr})\end{array}$ \\
\hline HIP $84840 \mathrm{~B}^{2}$ & $\ldots$ & $172022.02+201701.1$ & G1.5 & M4 & $13.852 \pm 0.007$ & $12.508 \pm 0.024$ & {$\left[112_{-15}^{+19}\right]$} & 16.9 & 1890 & & $0.1-\sim 10^{\mathrm{y}}$ \\
\hline HIP 85365 B & $\ldots$ & $172622.29-050211.2$ & F3 & L5.5 & $18.55 \pm 0.045$ & $16.693 \pm 0.168$ & {$\left[30.1_{-0.2}^{+0.2}\right]$} & 294.1 & 8850 & -0.09 & $1.6-1.9^{\mathrm{j}}$ \\
\hline HIP $86722 \mathrm{~B}^{\text {†b }}$ & $17433+2137 \mathrm{~B}$ & $174315.32+213604.2$ & K0 & M5.5 & $12.971 \pm 0.018$ & $11.511 \pm 0.025$ & {$\left[22.7_{-0.5}^{+0.4}\right]$} & 22.8 & 520 & -0.33 & $3.0-11.8^{\mathrm{j}}$ \\
\hline HIP 88728 B & $\ldots$ & $180650.92+085224.0$ & F5 & $\mathrm{DA}$ & $12.571 \pm 0.019$ & $10.269 \pm 0.097$ & {$\left[40.4_{-1.1}^{+1.2}\right]$} & 9.6 & 316 & $-0.1^{\circ}$ & $1.5-\sim 10^{y}$ \\
\hline HIP $90273 \mathrm{~B}^{\dagger}$ & $18251+3016 \mathrm{~B}$ & $182511.57+301643.2$ & K7 & M3.5 & $12.891 \pm 0.054$ & $11.56 \pm 0.022$ & {$\left[55.4_{-7.3}^{+10.0}\right]$} & 26.9 & 1490 & $\ldots$ & $0.3-\sim 10^{\mathrm{z}}$ \\
\hline HIP 90869 B & $\ldots$ & $183211.05-262945.1$ & G2 & M2 & $12.428 \pm 0.042$ & $11.101 \pm 0.029$ & {$\left[56.4_{-3.0}^{+3.4}\right]$} & 17.5 & 990 & $\begin{array}{l}-0.46 \\
1.9-5.7^{\mathrm{k}}\end{array}$ & $5.3-10.8^{\mathrm{j}}$ \\
\hline HIP 93967 B & $\ldots$ & $190756.43-151416.3$ & F9 & M3.5 & $14.529 \pm 0.007$ & $13.357 \pm 0.03$ & {$\left[122.1_{-19.6}^{+29.0}\right]$} & 22.2 & 2710 & $\ldots$ & $0.2-\sim 10^{\mathrm{y}}$ \\
\hline HIP $97168 \mathrm{~B}^{\dagger}$ & $19450+5136 \mathrm{~B}$ & $194459.38+513531.3$ & G4 & M5.5 & $14.997 \pm 0.004$ & $13.679 \pm 0.027$ & {$\left[97.5_{-10.4}^{+13.1}\right]$} & 12.6 & 1230 & $\ldots$ & $0.1-\sim 10^{\mathrm{y}}$ \\
\hline HIP $98535 \mathrm{C}^{\ddagger f}$ & $20011+4816 \mathrm{E}$ & $200102.17+481627.8$ & F5 & M5.5 & $14.763 \pm 0.065$ & $13.252 \pm 0.031$ & {$\left[60.0_{-3.3}^{+3.8}\right]$} & 61.7 & 3700 & -0.02 & $0.6-13.2^{\mathrm{j}}$ \\
\hline HIP $102582 \mathrm{~B}^{\dagger}$ & $20473+1052 \mathrm{~B}$ & $204716.75+105145.1$ & K2 & M5 & $13.256 \pm 0.001$ & $11.963 \pm 0.033$ & {$\left[31.3_{-1.3}^{+1.4}\right]$} & 14.7 & 459 & $\ldots$ & $0.2-\sim 10^{\mathrm{y}}$ \\
\hline HIP $103199 \mathrm{~B}^{\dagger}$ & $20547+3046 \mathrm{~B}$ & $205433.60+304540.6$ & G5 & M3.5 & $13.161 \pm 0.01$ & $11.817 \pm 0.027$ & {$\left[59.5_{-4.3}^{+5.1}\right]$} & 10.9 & 650 & $\ldots$ & $0.2-\sim 10^{\mathrm{y}}$ \\
\hline HIP $105202 \mathrm{~B}^{\dagger}$ & $21187+0857 \mathrm{~B}$ & $211842.31+085645.0$ & F5 & M4 & $13.456 \pm 0.019$ & $12.124 \pm 0.024$ & {$\left[41.8_{-1.3}^{+1.4}\right]$} & 89.4 & 3740 & -0.29 & $0.6-2.9^{\mathrm{j}}$ \\
\hline HIP 106551 B & $\ldots$ & $213445.17+383100.1$ & K3III & M5 & $14.597 \pm 0.01$ & $13.224 \pm 0.025$ & {$\left[71.0_{-2.1}^{+2.2}\right]$} & 66.1 & 4690 & $\ldots$ & $\leqslant 10^{\text {aa }}$ \\
\hline HIP $108822 \mathrm{~B}^{\dagger}$ & $22028+1207 \mathrm{~B}$ & $220248.15+120705.0$ & K7 & M3 & $12.079 \pm 0.002$ & $10.622 \pm 0.022$ & {$\left[44.4_{-6.1}^{+8.5}\right]$} & 31.8 & 1410 & $\ldots$ & $0.3-\sim 10^{\mathrm{z}}$ \\
\hline HIP 109454 B & $\ldots$ & $221027.58+304828.1$ & F5 & M3 & $13.729 \pm 0.116$ & $12.422 \pm 0.026$ & {$\left[100.9_{-5.8}^{+6.5}\right]$} & 18.2 & 1840 & -0.19 & $1.4-2.1^{\mathrm{j}}$ \\
\hline HIP $111657 \mathrm{~B}^{\dagger}$ & $22371+1159 \mathrm{~B}$ & $223708.65+115853.0$ & K7 & M4 & $11.978 \pm 0.041$ & $10.587 \pm 0.032$ & {$\left[41.9_{-3.0}^{+3.3}\right]$} & 7.8 & 330 & $\ldots$ & $0.3-\sim 10^{\mathrm{z}}$ \\
\hline 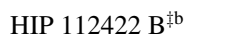 & $22463+3319 \mathrm{C}$ & $224618.57+331930.5$ & K2 & $\mathrm{L} 1.5$ & $17.776 \pm 0.021$ & $16.023 \pm 0.113$ & {$\left[65.4_{-6.6}^{+8.3}\right]$} & 16.0 & 1040 & $\ldots$ & $0.1-\sim 10^{* \mathrm{y}}$ \\
\hline HIP 114424 B & $\ldots$ & $231022.08-074854.7$ & G0 & M5.5 & $13.089 \pm 0.001$ & $11.595 \pm 0.026$ & {$\left[35.1_{-0.9}^{+1.1}\right]$} & 42.0 & 1480 & 0.08 & $0.6-4.5^{\mathrm{j}}$ \\
\hline & & & & & & & & & & 0.06 & $3.7-11.7^{\mathrm{i}}$ \\
\hline HIP $114456 \mathrm{~B}^{\dagger \ddagger \mathrm{a}}$ & $23108+4531 \mathrm{C}$ & $231054.78+453043.8$ & K0 & M5 & $12.371 \pm 0.003$ & $10.844 \pm 0.021$ & {$\left[24.3_{-0.3}^{+0.3}\right]$} & 50.4 & 1220 & 0.19 & $2.1-9.6^{\mathrm{k}}$ \\
\hline HIP 115819 B & $\ldots$ & $232749.76+045100.1$ & K5 & M8 & $16.865 \pm 0.022$ & $15.095 \pm 0.034$ & {$\left[65.7_{-8.2}^{+11.0}\right]$} & 30.4 & 2000 & $\ldots$ & $0.2-\sim 10^{\mathrm{y}}$ \\
\hline HIP $116052 \mathrm{~B}^{\dagger}$ & $23309+2747 \mathrm{~B}$ & $233052.38+274632.3$ & G5 & M3.5 & $13.718 \pm 0.002$ & $12.361 \pm 0.019$ & {$\left[49.6_{-2.3}^{+2.5}\right]^{[]}$} & 26.8 & 1330 & $\cdots$ & $0.3-\sim 10^{\mathrm{y}}$ \\
\hline \multicolumn{12}{|c|}{ Companions to faint non-HIP primaries } \\
\hline NLTT 1011 B & $\cdots$ & $001932.68+401855.4$ & K7 & $\mathrm{L} 2$ & $17.615 \pm 0.015$ & $15.544 \pm 0.059$ & $68.2_{-6.5}^{+6.6}$ & 58.5 & 3990 & $\cdots$ & $0.3-\sim 10^{\mathrm{z}}$ \\
\hline GD 280 B & $\ldots$ & $015458.13+481959.1$ & DA & M9 & $17.624 \pm 0.013$ & $16.034 \pm 0.082$ & $77_{-26}^{+35}$ & 63.4 & 4882 & $\ldots$ & $<10^{\text {aa }}$ \\
\hline NLTT 8245 B & $\ldots$ & $023323.52+654547.6$ & M0 & M7 & $16.465 \pm 0.007$ & $14.922 \pm 0.052$ & $53.0_{-7.5}^{+8.7}$ & 10.6 & 562 & $\ldots$ & $0.3-\sim 10^{\mathrm{z}}$ \\
\hline LSPM J0241+2553 B & $\ldots$ & $024151.51+255343.4$ & WD & $\mathrm{L} 1$ & $19.074 \pm 0.05$ & $17.027 \pm 0.180$ & $69_{-23}^{+35}$ & 31.2 & 2153 & $\ldots$ & $<10^{\text {aa }}$ \\
\hline HD 253662 B & $\ldots$ & $061353.35+151404.4$ & G8IV & L0.5 & $17.691 \pm 0.02$ & $15.961 \pm .0082$ & $>62.3$ & 20.1 & $>1252$ & $\ldots$ & $<10^{\text {aa }}$ \\
\hline LSPMJ0632+5053 B & $\ldots$ & $063248.55+505333.6$ & $\mathrm{G} 2$ & $\mathrm{~L} 1.5$ & $18.86 \pm 0.04$ & $16.383 \pm 0.108$ & $95.0_{-13.9}^{+16.3}$ & 47.4 & 4499 & $\ldots$ & $0.2-\sim 10^{y}$ \\
\hline NLTT 18587 B & $\ldots$ & $075413.38+372142.6$ & M2 & M7.5 & $18.03 \pm 0.026$ & $16.894 \pm 0.199$ & $132_{-31}^{+40}$ & 92.4 & 12200 & $\ldots$ & $0.3-\sim 1^{\mathrm{z}}$ \\
\hline NLTT 19109 B & $\ldots$ & $081159.00-085801.6$ & M4 & M8.5 & $18.36 \pm 0.044$ & $16.933 \pm 0.170$ & $132_{-36}^{+49}$ & 7.5 & 362 & $\ldots$ & $0.3-\sim 10^{\mathrm{z}}$ \\
\hline NLTT $22073 \mathrm{~B}^{\mathrm{g}}$ & $\ldots$ & $093337.11-275245.4$ & M2 & M7.5 & $16.303 \pm 0.007$ & $14.671 \pm 0.037$ & $29.9_{-6.9}^{+12.7}$ & 26 & 776 & $\ldots$ & $0.4-\sim 10^{\mathrm{x}}$ \\
\hline NLTT 23716 B & $\ldots$ & $101317.05-060122.9$ & $\mathrm{~K} 7$ & M8 & $15.884 \pm 0.006$ & $14.300 \pm 0.036$ & $48.2_{-6.8}^{+7.9}$ & 15.0 & 723 & $\ldots$ & $0.3-\sim 10^{\mathrm{z}}$ \\
\hline NLTT 26746 B & $\ldots$ & $111501.22+160700.8$ & M4 & L4 & $18.36 \pm 0.06$ & $16.403 \pm 0.118$ & $41.0_{-10.3}^{+12.2}$ & 18.0 & 661 & $\ldots$ & $0.3-\sim 10^{\mathrm{z}}$ \\
\hline NLTT 29395 B & $\ldots$ & $120302.61+580602.4$ & M3 & M8 & $17.397 \pm 0.013$ & $15.810 \pm 0.080$ & $56.4_{-13.0}^{+16.9}$ & 11.9 & 671 & $\ldots$ & $0.3-\sim 10^{\mathrm{z}}$ \\
\hline NLTT $30510 \mathrm{~B}$ & $\ldots$ & $122218.59+364348.1$ & M2 & M9.5 & $17.716 \pm 0.016$ & $15.971 \pm 0.080$ & $49.1_{-11.3}^{+14.8}$ & 19.6 & 962 & $\cdots$ & $0.3-\sim 10^{z}$ \\
\hline NLTT 31450 B & $\ldots$ & $123949.19+320903.1$ & M4 & L6 & $18.34 \pm 0.03$ & $16.135 \pm 0.105$ & $34.6_{-8.7}^{+10.3}$ & 12.3 & 487 & $\ldots$ & $0.3-\sim 10^{z}$ \\
\hline PMI 13410+0542 B & $\ldots$ & $134102.39+054248.8$ & M1 & L4 & $18.00 \pm 0.02$ & $16.187 \pm 0.143$ & $51.3_{-11.8}^{+21.7}$ & 9.4 & 484 & $\cdots$ & $0.3-\sim 10^{z}$ \\
\hline PMI 13518+4157 B & $\ldots$ & $135147.41+415747.4$ & M2.5 & $\mathrm{L} 1.5$ & $17.17 \pm 0.01$ & $15.081 \pm 0.032$ & $43.1_{-8.8}^{+9.9}$ & 21.6 & 613 & $\ldots$ & $0.3-\sim 10^{\mathrm{z}}$ \\
\hline
\end{tabular}


Table 2

(Continued)

\begin{tabular}{|c|c|c|c|c|c|c|c|c|c|c|c|}
\hline Object & $\begin{array}{c}\text { WDS } \\
\text { Designation }\end{array}$ & $\begin{array}{c}\text { Position } \\
(\text { Eq. }=\mathrm{J} 2000 \text { Ep. }=2010.0)\end{array}$ & $\begin{array}{c}\text { SpT } \\
\text { Primary }\end{array}$ & $\begin{array}{c}\text { SpT } \\
\text { Secondary }\end{array}$ & $\begin{array}{c}y_{P 1} \\
(\mathrm{mag})\end{array}$ & $\begin{array}{c}J_{2 \mathrm{MASS}} \\
(\mathrm{mag})\end{array}$ & $\begin{array}{c}d_{\text {primary }} \\
(\mathrm{pc})\end{array}$ & $\begin{array}{l}\text { Separation } \\
\left({ }^{\prime \prime}\right)\end{array}$ & $\begin{array}{l}\text { Separation } \\
\text { (AU) }\end{array}$ & $\begin{array}{l}\text { Metallicity }{ }^{\mathrm{k}} \\
\quad(\mathrm{dex})\end{array}$ & $\begin{array}{l}\text { Age } \\
(\mathrm{Gyr})\end{array}$ \\
\hline NLTT 38489 B & $\ldots$ & $144846.28+561057.8$ & M4 & M9 & $18.62 \pm 0.04$ & $16.64 \pm 0.143$ & $62.3_{-17.0}^{+23.3}$ & 6.7 & 418 & $\ldots$ & $1.2-\sim 10^{\mathrm{x}}$ \\
\hline NLTT 39312 B & $\ldots$ & $150559.27+333930.2$ & M2 & M8 & $17.768 \pm 0.018$ & $16.09 \pm 0.096$ & $140.9_{-32.7}^{+42.6}$ & 5.1 & 713 & $\ldots$ & $0.3-\sim 10^{\mathrm{z}}$ \\
\hline LSPM J1627+3328 B & $\ldots$ & $162701.44+332821.4$ & K7 & M9 & $16.386 \pm 0.018$ & $14.362 \pm 0.083$ & $38.0_{-5.4}^{+6.3}$ & 9.0 & 341 & $\ldots$ & $0.3-\sim 10^{\mathrm{z}}$ \\
\hline NLTT 44368 B & $\ldots$ & $171157.29+543043.8$ & M3 & $\mathrm{L} 1.5$ & $16.754 \pm 0.016$ & $14.736 \pm 0.058$ & $55.0_{-11.3}^{+12.7}$ & 90.2 & 7760 & $\ldots$ & $0.3-\sim 10^{z}$ \\
\hline LSPM J1717+5925 B & $\ldots$ & $171730.94+592530.2$ & G6 & M9 & $18.043 \pm 0.015$ & $16.580 \pm 0.168$ & $108.0_{-26.4}^{+34.8}$ & 14.4 & 1555 & $\ldots$ & $0.2-\sim 10^{\mathrm{y}}$ \\
\hline NLTT 52268 B & $\ldots$ & $215107.23+304554.7$ & M3 & M9 & $16.67 \pm 0.03$ & $14.945 \pm 0.052$ & $37.3_{-8.7}^{+11.3}$ & 14.7 & 549 & $\ldots$ & $0.3-\sim 10^{\mathrm{z}}$ \\
\hline LSPM J2153+1157 B & $\ldots$ & $215346.92+115746.4$ & M3 & M7 & $16.005 \pm 0.004$ & $14.403 \pm 0.038$ & $36.2_{-8.4}^{+10.9}$ & 11.3 & 408 & $\ldots$ & $0.3-\sim 10^{\mathrm{z}}$ \\
\hline PM I22118-1005 B & $\ldots$ & $221141.25-100820.8$ & M2 & L1.5 & $17.412 \pm 0.010$ & $15.246 \pm 0.049$ & $37.4_{-7.7}^{+8.6}$ & 204.5 & 8892 & $\ldots$ & $0.3-\sim 10^{\mathrm{z}}$ \\
\hline NLTT 55219 B & $\ldots$ & $225428.58+220256.1$ & M2 & L5.5 & $18.791 \pm 0.039$ & $16.313 \pm 0.122$ & $44.7_{-10.4}^{+13.5}$ & 9.7 & 432 & $\cdots$ & $0.3-\sim 10^{z}$ \\
\hline \multicolumn{12}{|c|}{ Serendipitous Companion Discoveries } \\
\hline NLTT 730 B & $\ldots$ & $001502.40+295929.8$ & M4 & $\mathrm{L} 7.5 \mathrm{p}^{\mathrm{d}}$ & $18.37 \pm 0.06$ & $16.16 \pm 0.08$ & $21.7_{-5.3}^{+8.1}$ & 233.6 & 5070 & $\ldots$ & $3-\sim 10^{2}$ \\
\hline NLTT 27966 B & $\ldots$ & $113639.44+485243.0$ & M5 & L4 & $18.48 \pm 0.05$ & $16.155 \pm 0.103$ & $39.6_{-10.2}^{+13.3}$ & 15.9 & 630 & $\ldots$ & $0.3-\sim 10^{z}$ \\
\hline LSPMJ1336+2541 B & $\ldots$ & $133624.89+254037.2$ & M3 & $\mathrm{L} 4$ & $19.45 \pm 0.09$ & $16.863 \pm 0.16$ & $60.7_{-13.9}^{+15.7}$ & 121.7 & 8793 & $\ldots$ & $0.3-\sim 10^{\mathrm{z}}$ \\
\hline HIP $73169 \mathrm{~B}^{\mathrm{e}}$ & $\ldots$ & $145711.35-061927.4$ & M0 & L2.5 & $18.42 \pm 0.09$ & $16.007 \pm 0.09$ & {$\left[27.3_{-6.3}^{+8.3}\right]$} & 29.1 & 796 & $\ldots$ & $0.3-\sim 10^{z}$ \\
\hline PM I23492+3458 B & $\ldots$ & $234915.11+345855.3$ & M2 & L9 & $18.80 \pm 0.04$ & $16.298 \pm 0.105$ & $30.7_{-6.3}^{+7.1}$ & 34.9 & 949 & $\ldots$ & $0.3-\sim 10^{2}$ \\
\hline \multicolumn{12}{|c|}{ Unlikely Companions } \\
\hline NLTT 35593 B & $\ldots$ & $135434.92-060734.3$ & M2 & $\mathrm{L} 2$ & $18.56 \pm 0.03$ & $16.299 \pm 0.114$ & $63.0_{-14.6}^{+19.1}$ & 1105.8 & 69706 & $\ldots$ & $0.3-\sim 10^{\triangleleft z}$ \\
\hline
\end{tabular}

Notes.

[] Denotes distance from trigonometric parallax; all other distances are photometric.

${ }^{\dagger}$ Previously known in the Washington Double Star Catalog.

¥ Additional possibly spurious companion in the system in the Washington Double Star Catalog.

* Simultaneously discovered by Allen et al. (2012).

a Our object appears in the Washington Double Star Catalog as component C due to a spurious companion listed as B.

${ }^{\mathrm{b}}$ Primary is itself a close double listed as Aa and Ab in the Washington Double Star Catalog.

${ }^{c}$ The companion was identified as a visual double during IRTF acquisition; due to saturation, it does not appear in our PS1 catalog. Position is taken from VISTA image

${ }^{\mathrm{d}}$ Spectral type from Kirkpatrick et al. (2010). We identify this object as a companion to NLTT 730

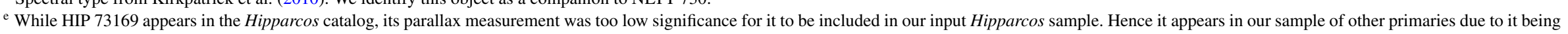
a nearby M dwarf in the proper motion catalog of Salim \& Gould (2003).

${ }^{\mathrm{f}}$ Primary is itself a binary with a listing in the Washington Double Star Catalog. Our companion is component C.

g Previously identified by Deacon \& Hambly (2007) as a binary pair. The secondary is also known as SIPS 0933-2752

${ }^{\mathrm{h}}$ The 2MASS photometry is only an upper limit; photometry taken from UKIDSS Lawrence et al. (2007).

${ }^{\mathrm{i}}$ Valenti \& Fischer (2005)

${ }^{\mathrm{j}}$ Casagrande et al. (2011).

${ }^{\mathrm{k}}$ Metallicity definition depends on source; metallicities from ${ }^{\mathrm{i}}$ are $[\mathrm{M} / \mathrm{H}]$ while those from ${ }^{\mathrm{j}}$ are $[\mathrm{Fe} / \mathrm{H}]$.

${ }^{1}$ Estimated spectral types from spectral deblending. See Section 5.2.1.

${ }^{\mathrm{m}}$ Previously identified as a candidate companion by Pinfield et al. (2006).

${ }^{\circ}$ Metallicity from Lee et al. (2011).

${ }^{\mathrm{w}}$ Robinson et al. (2007).

${ }^{x}$ This work, minimum age calculated from lack of activity and the activity lifetimes of West et al. (2008), approximate maximum age from disk-like kinematics.

y This work, minimum age calculated from limiting X-ray flux and the relations of Mamajek \& Hillenbrand (2008), approximate maximum age from disk-like kinematics.

${ }^{\mathrm{z}}$ This work, minimum age is that from Shkolnik et al. (2009) for objects with no X-ray emission, approximate maximum age from disk-like kinematics.

aa This work, approximate maximum age from disk-like kinematics.

${ }^{1}$ Object primary is a spectroscopic binary.

${ }^{2}$ Previously identified in Lépine \& Bongiorno (2007) 
Table 3

PS1 Data of Our Wide Companions

\begin{tabular}{|c|c|c|c|c|c|c|c|}
\hline Object & $\begin{array}{c}\text { Position } \\
(\text { Eq. }=\mathrm{J} 2000 \text { Ep. }=2010.0)\end{array}$ & $\mathrm{SpT}$ & $\begin{array}{l}g_{P 1} \\
(\mathrm{mag})\end{array}$ & $\begin{array}{c}r_{P 1} \\
(\mathrm{mag})\end{array}$ & $\begin{array}{c}i_{P 1} \\
(\mathrm{mag})\end{array}$ & $\begin{array}{l}z P 1 \\
(\mathrm{mag})\end{array}$ & $\begin{array}{c}y_{P 1} \\
(\mathrm{mag})\end{array}$ \\
\hline \multicolumn{8}{|c|}{ HIP search companions } \\
\hline HIP 2397 B & $003024.94+224447.1$ & L0.5 & & $\ldots$ & $19.085 \pm 0.013$ & $17.49 \pm 0.022$ & $16.606 \pm 0.008$ \\
\hline HIP $6217 \mathrm{C}$ & $011944.74+000618.5$ & M9.5 & $21.656 \pm 0.11$ & $21.076 \pm 0.071$ & $18.6 \pm 0.019$ & $17.444 \pm 0.007$ & $16.859 \pm 0.009$ \\
\hline HIP 6407 B & $012216.99+033122.0$ & $\mathrm{~L} 1+\mathrm{T}^{\mathrm{d}}$ & $\ldots$ & $\ldots$ & $20.094 \pm 0.042$ & $18.534 \pm 0.015$ & $17.527 \pm 0.027$ \\
\hline HIP $9269 \mathrm{~B}^{\ddagger}$ & $015910.98+331227.8$ & L6 & $\ldots$ & $\ldots$ & $\ldots$ & $\ldots$ & $18.266 \pm 0.031$ \\
\hline HIP 10033 B & $020910.58-021919.0$ & M5 & $19.709 \pm 0.017$ & $18.297 \pm 0.007$ & $16.456 \pm 0.003$ & $15.54 \pm 0.003$ & $15.14 \pm 0.003$ \\
\hline HIP 11161 B & $022336.59+524005.9$ & L1.5 & $\ldots$ & $\ldots$ & $21.24 \pm 0.095$ & $19.475 \pm 0.051$ & $18.785 \pm 0.067$ \\
\hline HIP 13589 B* $^{*}$ & $025507.10+213621.1$ & M7.5 & $\ldots$ & $\ldots$ & $\ldots$ & $18.003 \pm 0.012$ & $17.098 \pm 0.009$ \\
\hline HIP 26653 & $053949.51+525358.3$ & L5 & $\ldots$ & $20.542 \pm 0.043$ & & $17.719 \pm 0.011$ & $16.905 \pm 0.012$ \\
\hline HIP 32728 B & $064930.59+362626.0$ & M6.5 & $\ldots$ & $21.26 \pm 0.08$ & $18.057 \pm 0.006$ & $16.721 \pm 0.007$ & $16.060 \pm 0.006$ \\
\hline HIP $37283 \mathrm{~B}^{\dagger}$ & $073932.03+503107.3$ & M6 & $18.985 \pm 0.01$ & $17.622 \pm 0.006$ & $15.593 \pm 0.002$ & $14.639 \pm 0.001$ & $14.214 \pm 0.002$ \\
\hline HIP 46984 B & $093433.18+033407.0$ & M4 & $18.199 \pm 0.006$ & $17.038 \pm 0.004$ & $15.444 \pm 0.003$ & $14.848 \pm 0.002$ & $14.521 \pm 0.002$ \\
\hline HIP $49046 \mathrm{~B}^{\dagger}$ & $100035.71+271706.7$ & M6.5 & $20.127 \pm 0.021$ & $18.7 \pm 0.007$ & $16.282 \pm 0.002$ & $15.138 \pm 0.002$ & $14.482 \pm 0.002$ \\
\hline HIP 51877 B & $103554.72+365541.0$ & M9.5 & $\ldots$ & $20.23 \pm 0.039$ & $19.207 \pm 0.011$ & $17.801 \pm 0.004$ & $16.935 \pm 0.008$ \\
\hline HIP 52463 B & $104326.65-185218.2$ & M5 & $19.576 \pm 0.018$ & $18.25 \pm 0.02$ & $16.599 \pm 0.011$ & $15.479 \pm 0.001$ & $15.043 \pm 0.003$ \\
\hline HIP $55666 \mathrm{~B}^{\ddagger}$ & $112423.43-055409.8$ & M5.5 & $\ldots$ & $\ldots$ & $\ldots$ & & $14.533 \pm 0.037$ \\
\hline HIP $58918 \mathrm{~B}^{\dagger}$ & $120452.15+343723.2$ & M7 & $21.445 \pm 0.092$ & $20.122 \pm 0.026$ & $17.623 \pm 0.006$ & $16.491 \pm 0.004$ & $15.849 \pm 0.004$ \\
\hline HIP $59310 \mathrm{~B}^{\dagger}$ & $121009.79+185807.9$ & M7 & $21.021 \pm 0.052$ & $19.579 \pm 0.012$ & $17.077 \pm 0.005$ & $15.88 \pm 0.002$ & $15.252 \pm 0.002$ \\
\hline HIP 59933 B & $121736.41+142711.7$ & $\mathrm{~L} 1$ & 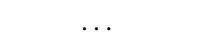 & $\ldots$ & $\ldots$ & $19.079 \pm 0.025$ & $18.111 \pm 0.032$ \\
\hline HIP $60501 \mathrm{~B}^{\dagger}$ & $122409.68+035603.3$ & M5 & $17.074 \pm 0.004$ & $15.814 \pm 0.002$ & $14.207 \pm 0.038$ & $13.313 \pm 0.001$ & $12.876 \pm 0.022$ \\
\hline HIP $60987 \mathrm{~B}^{\dagger \mathrm{c}}$ & $123001.60-060123.7$ & K7 & & & & & \\
\hline HIP $60987 \mathrm{C}^{\dagger}$ & $123000.68-060117.9$ & M3 & $15.395 \pm 0.005$ & $14.336 \pm 0.001$ & $\ldots$ & $12.825 \pm 0.001$ & $12.553 \pm 0.001$ \\
\hline HIP $63506 \mathrm{C}^{\ddagger}$ & $130050.27+421447.6$ & $\mathrm{~L} 1$ & $\ldots$ & $22.019 \pm 0.122$ & $19.49 \pm 0.014$ & $17.964 \pm 0.007$ & $16.999 \pm 0.008$ \\
\hline HIP $65706 \mathrm{~B}^{\dagger}$ & $132820.77+300317.0$ & M7 & $\ldots$ & $\ldots$ & $\ldots$ & & $15.016 \pm 0.003$ \\
\hline HIP $65780 C^{f}$ & $132906.09+112752.2$ & M5 & $18.523 \pm 0.001$ & $17.393 \pm 0.001$ & $15.669 \pm 0.001$ & $14.724 \pm 0.001$ & $14.327 \pm 0.001$ \\
\hline HIP $70623 \mathrm{C}^{\dagger}$ & $142645.74-051020.9$ & M5.5 & $17.424 \pm 0.008$ & $16.233 \pm 0.013$ & $14.762 \pm 0.061$ & $13.981 \pm 0.001$ & $13.619 \pm 0.003$ \\
\hline HIP 75310 B & $152310.01-102858.4$ & M5 & $20.018 \pm 0.062$ & & $15.647 \pm 0.003$ & $15.055 \pm 0.003$ & $14.668 \pm 0.003$ \\
\hline HIP $76456 \mathrm{~B}^{\dagger}$ & $153656.27+295931.1$ & M6.5 & $19.827 \pm 0.017$ & $19.027 \pm 0.006$ & $16.513 \pm 0.002$ & $15.343 \pm 0.002$ & $14.753 \pm 0.002$ \\
\hline HIP 76641 B & $153859.53+435217.2$ & M3.5 & $\ldots$ & $\ldots$ & $17.225 \pm 0.036$ & $13.026 \pm 0.001$ & $12.158 \pm 0.001$ \\
\hline HIP 78184 B & $155755.32+591425.3$ & M9 & $\ldots$ & $21.043 \pm 0.054$ & $18.642 \pm 0.014$ & $17.167 \pm 0.013$ & $16.219 \pm 0.024$ \\
\hline HIP $78859 \mathrm{~B}^{\dagger}$ & $160549.62+345953.9$ & M5 & $16.9 \pm 0.004$ & $15.633 \pm 0.024$ & $14.422 \pm 0.004$ & $13.832 \pm 0.009$ & $13.57 \pm 0.009$ \\
\hline HIP 78916 B & $160632.22+225334.5$ & M8 & $22.05 \pm 0.16$ & $19.694 \pm 0.021$ & $18.506 \pm 0.009$ & $17.354 \pm 0.006$ & $16.694 \pm 0.008$ \\
\hline HIP 78923 B & $160638.65+340619.3$ & M8 & $\ldots$ & $\ldots$ & $17.219 \pm 0.006$ & $16.187 \pm 0.002$ & $15.565 \pm 0.004$ \\
\hline HIP $79180 \mathrm{~B}^{\dagger}$ & $160942.06+654918.1$ & M5 & $20.008 \pm 0.02$ & $18.815 \pm 0.013$ & $16.718 \pm 0.003$ & $15.985 \pm 0.003$ & $15.315 \pm 0.003$ \\
\hline HIP $80258 \mathrm{~B}^{\dagger}$ & $162309.09+365050.2$ & M4 & $18.321 \pm 0.062$ & $16.993 \pm 0.023$ & $15.497 \pm 0.002$ & $14.782 \pm 0.111$ & $14.487 \pm 0.004$ \\
\hline HIP 81910 B & $164349.50-264840.2$ & M6 & $\ldots$ & $17.478 \pm 0.068$ & $15.494 \pm 0.012$ & $14.678 \pm 0.344$ & $13.852 \pm 0.025$ \\
\hline HIP 82233 B & $164803.98-155756.7$ & M5.5 & $\ldots$ & $15.693 \pm 0.003$ & $14.218 \pm 0.001$ & $\ldots$ & $13.198 \pm 0.009$ \\
\hline HIP $83651 \mathrm{~B}^{\dagger}$ & $170549.00+045724.8$ & M3 & $14.977 \pm 0.001$ & $13.978 \pm 0.035$ & $12.809 \pm 0.07$ & $12.018 \pm 0.001$ & $12.529 \pm 0.052$ \\
\hline HIP 84840 B & $172022.02+201701.1$ & M4 & $17.489 \pm 0.019$ & $16.272 \pm 0.008$ & $14.869 \pm 0.002$ & $14.126 \pm 0.014$ & $13.852 \pm 0.007$ \\
\hline HIP 85365 B & $172622.29-050211.2$ & L5.5 & $\ldots$ & $\ldots$ & $21.606 \pm 0.13$ & $19.547 \pm 0.05$ & $18.55 \pm 0.045$ \\
\hline HIP $86722 \mathrm{~B}^{\dagger}$ & $174315.32+213604.2$ & M5.5 & $\ldots$ & & $\ldots$ & $14.184 \pm 0.003$ & $12.971 \pm 0.018$ \\
\hline HIP 88728 B & $180650.92+085224.0$ & DA & $\ldots$ & $14.377 \pm 0.203$ & & $13.66 \pm 0.002$ & $12.571 \pm 0.019$ \\
\hline HIP 90273 B & $182511.57+301643.2$ & M3.5 & $16.348 \pm 0.003$ & $15.307 \pm 0.294$ & $13.905 \pm 0.066$ & $13.117 \pm 0.004$ & $12.891 \pm 0.054$ \\
\hline HIP 90869 B & $183211.05-262945.1$ & M2 & $15.375 \pm 0.024$ & $14.156 \pm 0.026$ & $\ldots$ & $12.443 \pm 0.001$ & $12.428 \pm 0.042$ \\
\hline HIP 93967 B & $190756.43-151416.3$ & M3.5 & $17.723 \pm 0.023$ & $\ldots$ & $15.433 \pm 0.028$ & $14.75 \pm 0.027$ & $14.529 \pm 0.007$ \\
\hline HIP 97168 B & $194459.38+513531.3$ & M5.5 & $18.5 \pm 0.014$ & $17.241 \pm 0.009$ & $16.081 \pm 0.007$ & $15.38 \pm 0.007$ & $14.997 \pm 0.004$ \\
\hline HIP $98535 C^{f}$ & $200102.17+481627.8$ & M5.5 & $19.513 \pm 0.046$ & $\ldots$ & $16.077 \pm 0.031$ & $15.183 \pm 0.057$ & $14.763 \pm 0.065$ \\
\hline HIP $102582 \mathrm{~B}^{\dagger}$ & $204716.75+105145.1$ & M5 & $17.437 \pm 0.003$ & $16.140 \pm 0.003$ & $14.408 \pm 0.001$ & $13.601 \pm 0.002$ & $13.256 \pm 0.001$ \\
\hline HIP $103199 \mathrm{~B}^{\dagger}$ & $205433.60+304540.6$ & M3.5 & $16.513 \pm 0.004$ & $\ldots$ & $15.03 \pm 0.912$ & $13.415 \pm 0.005$ & $13.161 \pm 0.01$ \\
\hline HIP $105202 \mathrm{~B}^{\dagger}$ & $211842.31+085645.0$ & M4 & $17.221 \pm 0.097$ & $15.867 \pm 0.031$ & $14.41 \pm 0.01$ & $13.718 \pm 0.002$ & $13.456 \pm 0.019$ \\
\hline HIP 106551 B & $213445.17+383100.1$ & M5 & $19.019 \pm 0.056$ & $17.677 \pm 0.034$ & $15.958 \pm 0.01$ & $14.979 \pm 0.01$ & $14.597 \pm 0.01$ \\
\hline HIP $108822 \mathrm{~B}^{\dagger}$ & $220248.15+120705.0$ & M3 & $15.019 \pm 0.007$ & $13.995 \pm 0.185$ & $13.459 \pm 0.451$ & $12.478 \pm 0.092$ & $12.079 \pm 0.002$ \\
\hline HIP 109454 B & $221027.58+304828.1$ & M3 & $16.605 \pm 0.004$ & & & $13.895 \pm 0.002$ & $13.729 \pm 0.116$ \\
\hline HIP $111657 \mathrm{~B}^{\dagger}$ & $223708.65+115853.0$ & M4 & $15.678 \pm 0.011$ & $14.389 \pm 0.015$ & $14.477 \pm 0.337$ & $\ldots$ & $11.978 \pm 0.041$ \\
\hline HIP $112422 B^{\ddagger b}$ & $224618.57+331930.5$ & $\mathrm{~L} 1.5$ & $\ldots$ & $\ldots$ & $19.899 \pm 0.041$ & $18.907 \pm 0.016$ & $17.776 \pm 0.021$ \\
\hline HIP $114424 \mathrm{~B}^{\dagger \ddagger}$ & $231022.08-074854.7$ & M5.5 & $\ldots$ & $16.296 \pm 0.004$ & $14.444 \pm 0.004$ & $13.548 \pm 0.076$ & $13.089 \pm 0.001$ \\
\hline HIP $114456 \mathrm{~B}^{\dagger \ddagger a}$ & $231054.78+453043.8$ & M5 & $17.26 \pm 0.054$ & $\ldots$ & $14.055 \pm 0.001$ & $13.063 \pm 0.001$ & $12.371 \pm 0.003$ \\
\hline HIP 115819 B & $232749.76+045100.1$ & M8 & $\ldots$ & $\ldots$ & $19.272 \pm 0.023$ & 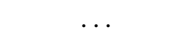 & $16.865 \pm 0.022$ \\
\hline HIP $116052 \mathrm{~B}^{\ddagger}$ & $233052.38+274632.3$ & M3.5 & $\ldots$ & $\ldots$ & $\ldots$ & $14.013 \pm 0.02$ & $13.718 \pm 0.002$ \\
\hline \multicolumn{8}{|c|}{ Companions to faint non-HIP primaries } \\
\hline NLTT $1011 \mathrm{~B}$ & $001932.68+401855.4$ & $\mathrm{~L} 2$ & $\cdots$ & $\cdots$ & $20.203 \pm 0.034$ & $18.651 \pm 0.013$ & $17.615 \pm 0.015$ \\
\hline GD 280 & $015458.13+481959.1$ & M9 & $\ldots$ & & $19.915 \pm 0.017$ & $18.524 \pm 0.017$ & $17.624 \pm 0.013$ \\
\hline NLTT 8245 & $023323.52+654547.6$ & M7 & $\ldots$ & $\ldots$ & $18.689 \pm 0.015$ & $17.362 \pm 0.005$ & $16.465 \pm 0.007$ \\
\hline LSPM J0241+2553 & $024151.51+255343.4$ & $\mathrm{~L} 1$ & $\ldots$ & $\ldots$ & $21.357 \pm 0.132$ & $19.986 \pm 0.065$ & $19.074 \pm 0.05$ \\
\hline
\end{tabular}


Table 3

(Continued)

\begin{tabular}{|c|c|c|c|c|c|c|c|}
\hline Object & $\begin{array}{c}\text { Position } \\
(\text { Eq. }=\mathrm{J} 2000 \text { Ep. }=2010.0)\end{array}$ & SpT & $\begin{array}{l}g_{P 1} \\
(\mathrm{mag})\end{array}$ & $\begin{array}{c}r_{P 1} \\
(\mathrm{mag})\end{array}$ & $\begin{array}{c}i_{P 1} \\
(\mathrm{mag})\end{array}$ & $\begin{array}{c}z_{P 1} \\
(\mathrm{mag})\end{array}$ & $\begin{array}{c}y_{P 1} \\
(\mathrm{mag})\end{array}$ \\
\hline HD 253662 & $061353.35+151404.4$ & L0.5 & $\ldots$ & $21.39 \pm 0.109$ & $20.306 \pm 0.073$ & $18.692 \pm 0.022$ & $17.691 \pm 0.02$ \\
\hline LSPMJ0632+5053 B & $063248.55+505333.6$ & L1.5 & $\ldots$ & $\ldots$ & $\ldots$ & $19.86 \pm 0.12$ & $18.86 \pm 0.04$ \\
\hline NLTT 18587 & $075413.38+372142.6$ & M7.5 & $\ldots$ & $\ldots$ & $20.507 \pm 0.057$ & $18.879 \pm 0.019$ & $18.03 \pm 0.026$ \\
\hline NLTT 19109 & $081159.00-085801.6$ & M8.5 & $\ldots$ & $\ldots$ & $20.679 \pm 0.042$ & $19.193 \pm 0.023$ & $18.36 \pm 0.044$ \\
\hline NLTT 22073 B & $093337.11-275245.4$ & M7.5 & $\ldots$ & $21.185 \pm 0.047$ & $18.619 \pm 0.007$ & $17.115 \pm 0.006$ & $16.303 \pm 0.007$ \\
\hline NLTT 23716 & $101317.05-060122.9$ & M8 & $22.289 \pm 0.221$ & $\ldots$ & $17.995 \pm 0.006$ & $16.703 \pm 0.005$ & $15.884 \pm 0.006$ \\
\hline NLTT 26746 B & $111501.22+160700.8$ & L4 & $\ldots$ & $\ldots$ & $21.23 \pm 0.07$ & $19.43 \pm 0.05$ & $18.36 \pm 0.06$ \\
\hline NLTT 29395 & $120302.61+580602.4$ & M8 & $\ldots$ & $\ldots$ & $19.605 \pm 0.018$ & $18.181 \pm 0.008$ & $17.397 \pm 0.013$ \\
\hline NLTT 30510 & $122218.59+364348.1$ & M9.5 & $\ldots$ & $\ldots$ & $20.371 \pm 0.064$ & $18.657 \pm 0.014$ & $17.716 \pm 0.016$ \\
\hline NLTT $31450 \mathrm{~B}$ & $123949.19+320903.1$ & L6 & $\ldots$ & $\ldots$ & $20.8 \pm 0.09$ & $19.29 \pm 0.02$ & $18.34 \pm 0.03$ \\
\hline PMI 13410+0542 B & $134102.39+054248.8$ & L4 & $\ldots$ & $21.5 \pm 0.2$ & $20.10 \pm 0.05$ & $18.92 \pm 0.03$ & $18.00 \pm 0.02$ \\
\hline PMI 13518+4157 B & $135147.41+415747.4$ & $\mathrm{~L} 1.5$ & $\ldots$ & $21.64 \pm 0.13$ & $19.636 \pm 0.018$ & $18.190 \pm 0.009$ & $17.17 \pm 0.01$ \\
\hline NLTT 38489 B & $144846.28+561057.8$ & M9 & $\ldots$ & $\ldots$ & $20.88 \pm 0.05$ & $19.52 \pm 0.04$ & $18.62 \pm 0.04$ \\
\hline NLTT 39312 B & $150559.27+333930.2$ & M8 & $\ldots$ & $\ldots$ & $19.588 \pm 0.018$ & $18.476 \pm 0.017$ & $17.768 \pm 0.018$ \\
\hline LSPM J1627+3328 B & $162701.44+332821.4$ & M9 & $\ldots$ & $\ldots$ & $18.94 \pm 0.16$ & $17.32 \pm 0.03$ & $16.386 \pm 0.018$ \\
\hline NLTT 44368 B & $171157.29+543043.8$ & L1.5 & $\ldots$ & $21.28 \pm 0.07$ & 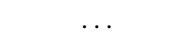 & $17.688 \pm 0.011$ & $16.754 \pm 0.016$ \\
\hline LSPM J1717+5925 B & $171730.94+592530.2$ & M9 & $\ldots$ & $\ldots$ & $20.232 \pm 0.053$ & $18.890 \pm 0.013$ & $18.043 \pm 0.015$ \\
\hline NLTT $52268 \mathrm{~B}$ & $215107.23+304554.7$ & M9 & $\ldots$ & $\ldots$ & $19.08 \pm 0.01$ & $17.63 \pm 0.01$ & $16.67 \pm 0.03$ \\
\hline LSPM J2153+1157 B & $215346.92+115746.4$ & M7 & $22.28 \pm 0.08$ & $20.90 \pm 0.03$ & $18.149 \pm 0.005$ & $16.787 \pm 0.003$ & $16.005 \pm 0.004$ \\
\hline PM I22118-1005 B & $221141.25-100820.8$ & $\mathrm{~L} 1.5$ & $\ldots$ & $22.28 \pm 0.14$ & $19.77 \pm 0.02$ & $18.347 \pm 0.010$ & $17.412 \pm 0.010$ \\
\hline NLTT $55219 \mathrm{~B}$ & $225428.58+220256.1$ & L5.5 & $\ldots$ & $\ldots$ & $\ldots$ & $19.665 \pm 0.04$ & $18.791 \pm 0.039$ \\
\hline \multicolumn{8}{|c|}{ Serendipitous Companion Discoveries } \\
\hline NLTT $730 \mathrm{~B}$ & $001502.40+295929.8$ & L7.5p ${ }^{\mathrm{d}}$ & $\ldots$ & $\ldots$ & $21.63 \pm 0.11$ & $19.49 \pm 0.04$ & $18.37 \pm 0.06$ \\
\hline NLTT 27966 B & $113639.44+485243.0$ & L4 & $\ldots$ & $\ldots$ & $21.06 \pm 0.13$ & $19.52 \pm 0.03$ & $18.48 \pm 0.05$ \\
\hline LSPMJ1336+2541 B & $133624.89+254037.2$ & L4 & $\ldots$ & $\ldots$ & $22.19 \pm 0.16$ & $20.21 \pm 0.07$ & $19.45 \pm 0.09$ \\
\hline HIP $73169 \mathrm{~B}^{\mathrm{e}}$ & $145711.35-061927.4$ & $\mathrm{~L} 2.5$ & $\ldots$ & $\ldots$ & $20.55 \pm 0.18$ & $19.06 \pm 0.02$ & $18.42 \pm 0.09$ \\
\hline PM I23492+3458 B & $234915.11+345855.3$ & L9 & $\ldots$ & $\ldots$ & $\ldots$ & $19.99 \pm 0.04$ & $18.80 \pm 0.04$ \\
\hline \multicolumn{8}{|c|}{ Unlikely Companions } \\
\hline NLTT 35593 B & $135434.92-060734.3$ & L2 & $\ldots$ & $\ldots$ & $20.74 \pm 0.12$ & $19.37 \pm 0.11$ & $18.56 \pm 0.03$ \\
\hline
\end{tabular}

Notes.

$\dagger$ Previously known in the Washington Double Star Catalog.

$\ddagger$ Additional possibly spurious companion in the system in the Washington Double Star Catalog.

* Simultaneously discovered by Allen et al. (2012).

${ }^{a}$ Our object appears in the Washington Double Star Catalog as component C due to a spurious companion listed as B.

${ }^{\mathrm{b}}$ Primary is itself a close double listed as Aa and $\mathrm{Ab}$ in the Washington Double Star Catalog.

${ }^{c}$ The companion was identified as visual double during IRTF acquisition, due to saturation, it does not appear in our PS1 catalog, position is taken from VISTA image.

${ }^{\mathrm{d}}$ Spectral type from Kirkpatrick et al. (2010). We identify this object as a companion to NLTT 730.

e While HIP 73169 appears in the Hipparcos catalog, its parallax measurement was too low-significance for it to be included in our input Hipparcos sample. Hence it appears in our sample of other primaries due to it being a nearby M dwarf in the proper motion catalog of Salim \& Gould (2003).

${ }^{\mathrm{f}}$ Primary is itself a binary with a listing in the Washington Double Star Catalog. Our companion is component C.

g Previously identified by Deacon \& Hambly (2007) as a binary pair. The secondary is also known as SIPS 0933-2752.

WFCAM Science Archive; Hambly et al. 2008) and Table 7 for mid-infrared photometry from WISE (Wright et al. 2010; Cutri et al. 2012).

\subsection{Near-infrared Spectroscopy}

To characterize our companions, we obtained $0.8-2.5 \mu \mathrm{m}$ spectroscopy using the SpeX instrument (Rayner et al. 2003) on the NASA Infrared Telescope (IRTF). To minimize the possibility of observing an unrelated background object due to a non-physical chance alignment with one of our primaries, we preferentially followed-up (i.e., we were more likely to follow-up) companions closer than five arcminutes but did not follow-up all of our candidate companions. In total 115 candidate companions to stars were passed to the IRTF queue, 87 are presented here as true companions, 1 we classify as an unlikely companion, 2 are candidate white dwarf companions not presented in this work, and the remaining 26 were not observed.

Depending on the brightness of the object and the weather conditions, we used either the low-resolution $(R \approx 75-120)$ single-order prism mode or the moderateresolution $(R \approx 750-2000)$ multiple-order cross-dispersed SXD mode. The slit width was chosen to match the seeing and was oriented along the parallactic angle to minimize atmospheric dispersion. The observations were taken in nodded ABBA patterns. The standards were taken contemporaneously with each science target and at similar airmass and sky position. We reduced all our spectra using version 3.4 of the SpeXtool software package (Cushing et al. 2004; Vacca et al. 2003). See Table 8 for details of the exposure times, weather conditions, and standard stars used for each object.

We spectrally classified our objects by visually comparing with the $\mathrm{M}$ and $\mathrm{L}$ near-infrared standards from Kirkpatrick 
Table 4

Astrometric Data on Our Data of Our Multiple Systems

\begin{tabular}{|c|c|c|c|c|c|c|c|c|}
\hline Object & $\begin{array}{c}d \\
(\mathrm{pc})\end{array}$ & $\begin{array}{l}\mu_{\alpha} \cos \delta \\
\left({ }^{\prime \prime} \mathrm{yr}^{-1}\right)\end{array}$ & $\begin{array}{c}\mu_{\delta} \\
\left({ }^{\prime \prime} \mathrm{yr}^{-1}\right)\end{array}$ & $\begin{array}{c}d \mu_{\alpha} \cos \delta \\
n_{\sigma}\end{array}$ & $\begin{array}{c}d \mu_{\delta} \\
n_{\sigma}\end{array}$ & $\begin{array}{l}d \mu \\
n_{\sigma}\end{array}$ & $\begin{array}{c}\text { Projected } \\
\text { Separation } \\
\left({ }^{\prime \prime}\right)\end{array}$ & $\begin{array}{c}\text { Projected } \\
\text { Separation } \\
\text { (AU) }\end{array}$ \\
\hline HIP 2397 & {$\left[33.9_{-1.3}^{+1.4}\right]$} & $0.186 \pm 0.001$ & $-0.185 \pm 0.001$ & 3.2 & 2.1 & 3.8 & 117.1 & 3970 \\
\hline HIP 2397B & $39.2_{-6.4}^{+7.6}$ & $0.2 \pm 0.004$ & $-0.176 \pm 0.004$ & & & & & \\
\hline HIP 6217 & {$\left[101_{-13}^{+18}\right]$} & $0.119 \pm 0.001$ & $0.030 \pm 0.001$ & 1.94 & 1.94 & 2.74 & 27.4 & 2767 \\
\hline HIP 6217 B & $70.9_{-11.7}^{+14.0}$ & $0.111 \pm 0.004$ & $0.022 \pm 0.004$ & & & & & \\
\hline HIP 6407 & {$\left[57.2_{-3.0}^{+3.3}\right]$} & $0.046 \pm 0.001$ & $-0.159 \pm 0.001$ & 2.1 & 2.7 & 3.4 & 44.9 & 2570 \\
\hline HIP 6407B & $53.6_{-8.8}^{+10.5}$ & $0.03 \pm 0.008$ & $-0.138 \pm 0.008$ & & & & & \\
\hline HIP 9269 & {$\left[25.0_{-0.4}^{+0.3}\right]$} & $0.244 \pm 0.001$ & $-0.352 \pm 0.001$ & 0.1 & 0.5 & 0.5 & 52.1 & 1300 \\
\hline HIP 9269B & $27.8_{-4.5}^{+5.4}$ & $0.243 \pm 0.011$ & $-0.347 \pm 0.011$ & & & & & \\
\hline HIP 10033 & {$\left[69.7_{-31}^{+3.4}\right]$} & $-0.093 \pm 0.001$ & $-0.046 \pm 0.001$ & 2.9 & 2.6 & 3.9 & 42.9 & 2990 \\
\hline HIP 10033B & $89.5_{-14.5}^{+17.3}$ & $-0.114 \pm 0.007$ & $-0.027 \pm 0.007$ & & & & & \\
\hline HIP 11161 & {$\left[69.3_{-3.6}^{+4.1}\right]$} & $-0.081 \pm 0.001$ & $-0.061 \pm 0.001$ & 0.4 & 0.6 & 0.7 & 47.7 & 3300 \\
\hline HIP 11161B & $83.6_{-13.6}^{+16.3}$ & $-0.079 \pm 0.005$ & $-0.058 \pm 0.005$ & & & & & \\
\hline HIP 13589 & {$\left[72.8_{-4.0}^{+4.6}\right]$} & $0.148 \pm 0.001$ & $-0.097 \pm 0.001$ & 0.5 & 1.7 & 1.8 & 49.2 & 3580 \\
\hline HIP 13589B & $64.6_{-10.5}^{+12.5}$ & $0.15 \pm 0.005$ & $-0.089 \pm 0.005$ & & & & & \\
\hline HIP 26653 & $27.9_{-0.7}^{+0.8}$ & $-0.013 \pm 0.001$ & $-0.142 \pm 0.001$ & 2.91 & 2.94 & 4.66 & 27.0 & 753 \\
\hline HIP 26653 B & $18.8_{-3.1}^{+3.1}$ & $-0.001 \pm 0.004$ & $-0.157 \pm 0.004$ & & & & & \\
\hline HIP 32728 & {$\left[46.6_{-2.6}^{+2.9}\right]$} & $0.191 \pm 0.001$ & $-0.14 \pm 0.001$ & 0.2 & 0.9 & 1.0 & 94.3 & 4400 \\
\hline HIP 32728B & $67.1_{-11.2}^{+13.4}$ & $0.188 \pm 0.016$ & $-0.13 \pm 0.011$ & & & & & \\
\hline HIP 37283 & {$\left[45.5_{-1.2}^{+1.2}\right]$} & $-0.08 \pm 0.001$ & $-0.061 \pm 0.001$ & 1.4 & 0.6 & 1.6 & 106.1 & 4830 \\
\hline HIP 37283B & $34.6_{-6.1}^{+7.4}$ & $-0.087 \pm 0.005$ & $-0.058 \pm 0.005$ & & & & & \\
\hline HIP 46984 & {$\left[74.7_{-6.9}^{+8.6}\right]$} & $0.026 \pm 0.002$ & $0.108 \pm 0.002$ & 1.6 & 1.8 & 2.4 & 37.5 & 2800 \\
\hline HIP 46984B & $111.9_{-18.6}^{+22.4}$ & $0.032 \pm 0.004$ & $0.101 \pm 0.003$ & & & & & \\
\hline HIP 49046 & {$\left[34.7_{-2.3}^{+2.6}\right]$} & $-0.015 \pm 0.002$ & $0.119 \pm 0.001$ & 1.5 & 1.1 & 1.9 & 136.1 & 4720 \\
\hline HIP 49046B & $33.3_{-5.6}^{+6.7}$ & $-0.008 \pm 0.005$ & $0.113 \pm 0.005$ & & & & & \\
\hline HIP 51877 & $54.2_{-2.1}^{+2.3}$ & $-0.063 \pm 0.001$ & $-0.122 \pm 0.001$ & 0.50 & 0.33 & 0.60 & 17.6 & 954 \\
\hline HIP 51877 B & $57.4_{-9.6}^{+11.5}$ & $-0.066 \pm 0.006$ & $-0.124 \pm 0.006$ & & & & & \\
\hline HIP 52463 & {$\left[74.2_{-4.5}^{+5.2}\right]$} & $-0.07 \pm 0.001$ & $0.083 \pm 0.001$ & 1.1 & 2.2 & 2.4 & 43.5 & 3230 \\
\hline HIP 52463B & $84.6_{-14.1}^{+16.9}$ & $-0.064 \pm 0.006$ & $0.096 \pm 0.006$ & & & & & \\
\hline HIP 55666 & {$\left[49.8_{-1.5}^{+1.5}\right]$} & $-0.085 \pm 0.001$ & $-0.093 \pm 0.001$ & 0.2 & 2.3 & 2.3 & 37.2 & 1850 \\
\hline HIP 55666B & $42.2_{-6.9}^{+8.3}$ & $-0.087 \pm 0.009$ & $-0.074 \pm 0.009$ & & & & & \\
\hline HIP 58918 & {$\left[55.8_{-5.1}^{+6.2}\right]$} & $-0.297 \pm 0.002$ & $0.018 \pm 0.001$ & 1.1 & 2.9 & 3.1 & 20.0 & 1110 \\
\hline HIP 58918B & $61.9_{-10.2}^{+12.3}$ & $-0.289 \pm 0.007$ & $0.038 \pm 0.007$ & & & & & \\
\hline HIP 59310 & {$\left[44.4_{-2.8}^{+3.2}\right]$} & $-0.157 \pm 0.002$ & $-0.041 \pm 0.001$ & 2.8 & 2.7 & 3.9 & 82.1 & 3650 \\
\hline HIP 59310B & $42.4_{-7.0}^{+8.4}$ & $-0.144 \pm 0.004$ & $-0.053 \pm 0.004$ & & & & & \\
\hline HIP 59933 & {$\left[57.0_{-2.4}^{+2.7}\right]$} & $-0.103 \pm 0.001$ & $-0.036 \pm 0.001$ & 2.0 & 1.0 & 2.3 & 38.1 & 2170 \\
\hline HIP 59933B & $67.8_{-11.3}^{+13.6}$ & $-0.088 \pm 0.007$ & $-0.029 \pm 0.007$ & & & & & \\
\hline HIP 60501 & {$\left[56.6_{-6.0}^{+7.6}\right]$} & $-0.012 \pm 0.002$ & $-0.134 \pm 0.001$ & 1.6 & 0.7 & 1.8 & 37.1 & 2100 \\
\hline HIP 60501B & $30.8_{-5.0}^{+6.0}$ & $-0.001 \pm 0.006$ & $-0.13 \pm 0.006$ & & & & & \\
\hline HIP 60987 & {$\left[61.9_{-2.3}^{+2.4}\right]$} & $-0.108 \pm 0.001$ & $-0.068 \pm 0.001$ & 1.0 & 1.5 & 1.8 & 19.0 & 1170 \\
\hline HIP 60987C & $64.6_{-10.8}^{+12.9}$ & $-0.104 \pm 0.004$ & $-0.074 \pm 0.004$ & & & & & \\
\hline HIP 63506 & {$\left[42.5_{-3.9}^{+4.7}\right]$} & $-0.396 \pm 0.002$ & $0.026 \pm 0.002$ & 0.5 & 0.0 & 0.5 & 132.8 & 5640 \\
\hline HIP $63506 \mathrm{C}$ & $45.0_{-7.5}^{+9.0}$ & $-0.401 \pm 0.009$ & $0.026 \pm 0.009$ & & & & & \\
\hline
\end{tabular}




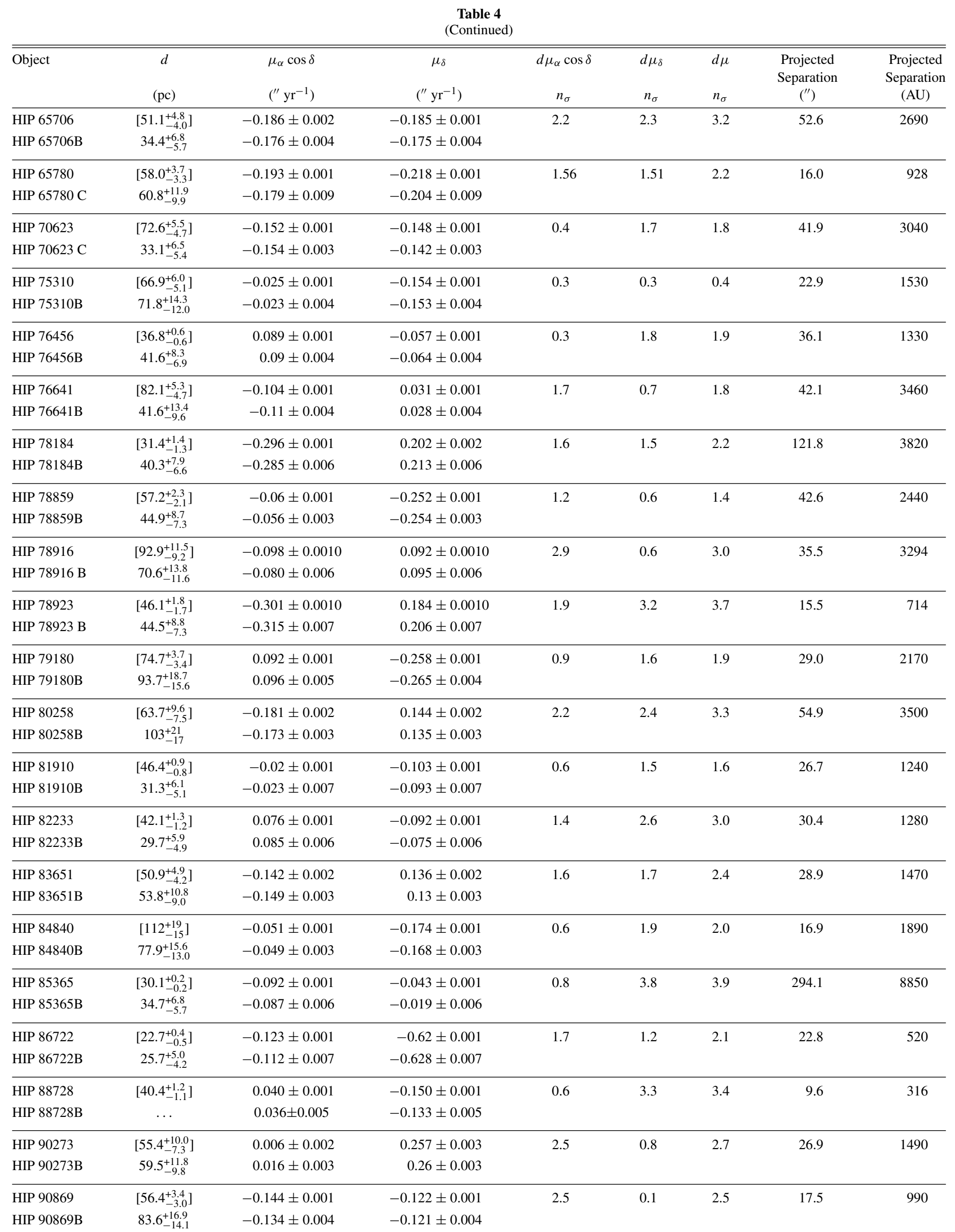




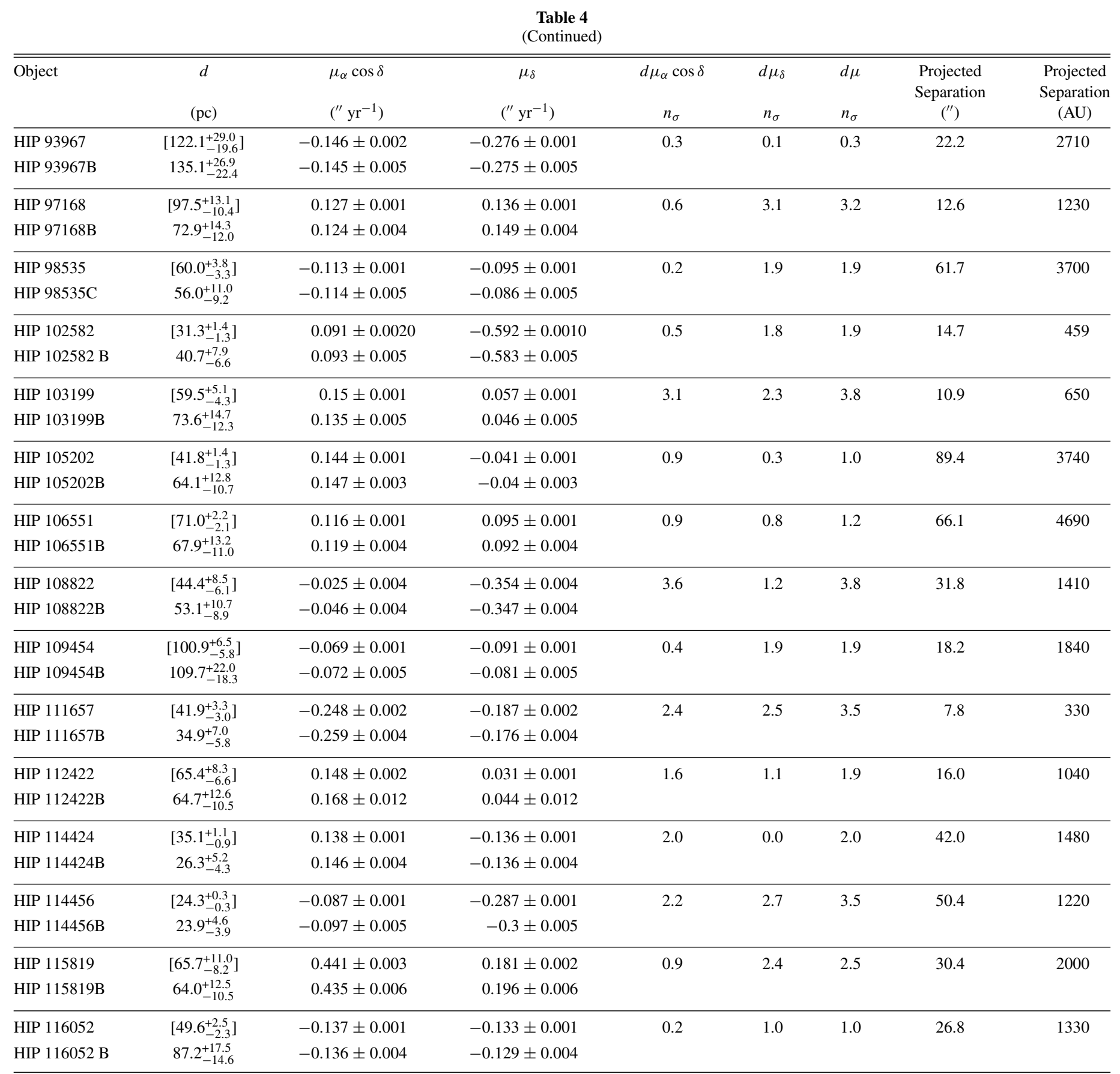

Notes. $n_{\sigma}$ is the number of standard deviations the proper motions differ by. [...] denotes distance from trigonometric parallax; all other distances are photometric.

et al. (2010). Additionally, for our L dwarf companions we measured three flux indices $\left(\mathrm{H}_{2} \mathrm{O}-\mathrm{J}, \mathrm{H}_{2} \mathrm{O}-\mathrm{H}\right.$, and $\left.\mathrm{CH}_{4}-\mathrm{K}\right)$ from Burgasser et al. (2006) relevant for L dwarfs. We then applied the polynomial relations of Burgasser (2007) to derive spectral types, averaging over the types derived from each index. We note that the $\mathrm{CH}_{4}-\mathrm{K}$ index does not depend strongly on spectral type earlier than mid-L and hence excluded this from the averaging for objects with this visual classification (see Table 9). Figures 1 and 2 show our early to mid-M dwarf companions to Hipparcos stars, Figure 3 shows our late-M and L dwarf Hipparcos companions, and Figure 4 shows our companions around non-Hipparcos primaries and serendipitous discoveries. Additionally, we observed a number of our primary stars that lacked spectral types in the literature. The spectra for these objects are shown in Figure 5.

For objects with spectral types earlier than $\mathrm{M}$, there are no near-infrared standards so we compared with the spectral library of Cushing et al. (2005) and Rayner et al. (2009), selecting the best comparison spectrum visually. For any of our objects with spectral types earlier than M5, we specifically examined the $\sim 0.85 \mu \mathrm{m}$ TiO feature in the $Z$-band and the $Y$-band $1 \mu \mathrm{m}$ $\mathrm{FeH}$ feature. For two primaries classified as $\mathrm{G}$ dwarfs we also examined the strengths of metal and hydrogen lines. We identified one of our companions (HIP 88728 B) as having blue continua and weak Paschen lines. Hence we classify this object as a DA white dwarf (see Figure 6). 
Table 5

The Data of Our Non-Hipparcos and Serendipitous Pairs

\begin{tabular}{|c|c|c|c|c|c|c|c|c|}
\hline Object & $\begin{array}{c}\text { distance } \\
(\mathrm{pc})\end{array}$ & $\begin{array}{l}\mu_{\alpha} \cos \delta \\
\left({ }^{\prime \prime} \mathrm{yr}^{-1}\right)\end{array}$ & $\begin{array}{c}\mu_{\delta} \\
\left({ }^{\prime \prime} \mathrm{yr}^{-1}\right)\end{array}$ & $\begin{array}{c}d \mu_{\alpha} \cos \delta \\
n_{\sigma}\end{array}$ & $\begin{array}{c}d \mu_{\delta} \\
n_{\sigma} \\
\end{array}$ & $\begin{array}{l}d \mu \\
n_{\sigma}\end{array}$ & $\begin{array}{l}\text { Projected } \\
\text { Separation } \\
\quad\left({ }^{\prime \prime}\right)\end{array}$ & $\begin{array}{c}\text { Projected } \\
\text { Separation } \\
(\mathrm{AU})\end{array}$ \\
\hline \multicolumn{9}{|c|}{ Other Companion Discoveries } \\
\hline NLTT 1011 & $68.2_{-6.5}^{+6.6 \mathrm{a}}$ & $-0.076 \pm 0.008^{f}$ & $-0.192 \pm 0.008$ & 0.08 & 0.43 & 0.44 & 58.5 & 3990 \\
\hline NLTT 1011B & $46.1_{-7.7}^{+9.3 b}$ & $-0.077 \pm 0.007$ & $-0.197 \pm 0.007$ & & & & & \\
\hline GD 280 & $77_{-26}^{+35}$ & $0.171 \pm 0.008$ & $-0.059 \pm 0.008^{f}$ & 0.32 & 0.53 & 0.62 & 63.4 & 4882 \\
\hline GD $280 \mathrm{~B}$ & $82.3_{-13.9}^{+16.7}$ & $0.174 \pm 0.005$ & $-0.054 \pm 0.005$ & & & & & \\
\hline NLTT 8245 & $53.0_{-7.5}^{+8.7}$ & $-0.096 \pm 0.008$ & $-0.164 \pm 0.008^{\mathrm{f}}$ & 0.42 & 0.21 & 0.47 & 10.6 & 562 \\
\hline NLTT 8245 B & $72.9_{-12.2}^{+14.6}$ & $-0.092 \pm 0.005$ & $-0.162 \pm 0.005$ & & & & & \\
\hline LSPM J0241+2553 & $69_{-23}^{+35}$ & $-0.032 \pm 0.008$ & $-0.158 \pm 0.008^{\mathrm{f}}$ & 1.41 & 0.70 & 1.58 & 31.2 & 2153 \\
\hline LSPM J0241+2553 B & $93.1_{-16.62}^{+20.2}$ & $-0.048 \pm 0.008$ & $-0.150 \pm 0.008$ & & & & & \\
\hline HD 253662 & $>62.3$ & $0.020 \pm 0.001$ & $-0.157 \pm 0.001^{\mathrm{i}}$ & 0.20 & 0.50 & 0.54 & 20.1 & $>1252$ \\
\hline HD 253662 B & $67.2_{-11.4}^{+13.7}$ & $-0.022 \pm 0.010$ & $-0.162 \pm 0.010$ & & & & & \\
\hline LSPM J0632+5053 & $95.0_{-13.9}^{+16.3 \mathrm{a}}$ & $0.035 \pm 0.008^{\mathrm{f}}$ & $-0.154 \pm 0.008$ & 2.1 & 1.2 & 2.4 & 47.4 & 4499 \\
\hline LSPM J0632+5053 B & $85.3_{-14.0}^{+16.7 \mathrm{~b}}$ & $0.054 \pm 0.004$ & $-0.143 \pm 0.004$ & & & & & \\
\hline NLTT 18587 & $132_{-31}^{+40}$ & $0.167 \pm 0.008$ & $-0.089 \pm 0.008^{\mathrm{f}}$ & 1.06 & 0.09 & 2.37 & 92.4 & 12200 \\
\hline NLTT 18587 B & $158_{-29}^{+36}$ & $0.155 \pm 0.008$ & $-0.0 .88 \pm 0.008$ & & & & & \\
\hline NLTT 19109 & $48.2_{-6.8}^{+7.9}$ & $0.035 \pm 0.008$ & $-0.181 \pm 0.008^{\mathrm{g}}$ & 1.59 & 1.9 & 2.48 & 7.5 & 362 \\
\hline NLTT 19109 B & $127_{-21}^{+25}$ & $0.050 \pm 0.005$ & $-0.163 \pm 0.005$ & & & & & \\
\hline NLTT 22073 & $29.9_{-6.9}^{+12.7 \mathrm{c}}$ & $-0.315 \pm 0.008$ & $0.16 \pm 0.008^{g}$ & 1.1 & 1.5 & 1.8 & 26 & 776 \\
\hline NLTT 22073 B & $62.2_{-10.4}^{+12.4}$ & $-0.301 \pm 0.014$ & $0.141 \pm 0.014$ & & & & & \\
\hline NLTT 23716 & $48.2_{-6.8}^{+7.9}$ & $0.054 \pm 0.005$ & $-0.286 \pm 0.005^{\mathrm{h}}$ & 1.41 & 1.41 & 1.99 & 15.0 & 723 \\
\hline NLTT 23716 B & $47.5_{-7.8}^{+9.3}$ & $0.063 \pm .004$ & $-0.277 \pm 0.004$ & & & & & \\
\hline NLTT 26746 & $41.0_{-10.3}^{+12.2 \mathrm{~d}}$ & $-0.251 \pm 0.008^{f}$ & $-0.147 \pm 0.008$ & 1.22 & 0.87 & 1.5 & 18.0 & 661 \\
\hline NLTT 26746 B & $42.5_{-6.9}^{+8.3 b}$ & $-0.235 \pm 0.01$ & $-0.136 \pm 0.01$ & & & & & \\
\hline NLTT 29395 & $56.4_{-13.0}^{+16.9}$ & $-0.182 \pm 0.008$ & $-0.115 \pm 0.008^{\mathrm{f}}$ & 2.01 & 0.78 & 2.16 & 11.9 & 671 \\
\hline NLTT 29395 B & $96.0_{-16.2}^{+19.5}$ & $-0.164 \pm 0.004$ & $-0.108 \pm 0.004$ & & & & & \\
\hline NLTT 30510 & $49.1_{-11.3}^{+14.8}$ & $0.215 \pm 0.008$ & $-0.067 \pm 0.008$ & ${ }^{\mathrm{f}} 0.4$ & 0.2 .5 & 2.53 & 19.6 & 962 \\
\hline NLTT 30510 B & $88.8_{-15.0}^{+18.1}$ & $0.219 \pm 0.006$ & $-0.042 \pm 0.006$ & & & & & \\
\hline NLTT 31450 & $34.6_{-8.7}^{+10.3 \mathrm{~d}}$ & $-0.034 \pm 0.008^{f}$ & $-0.202 \pm 0.008$ & -0.03 & 1.75 & 1.75 & 12.3 & 487 \\
\hline NLTT 31450 B & $27.2_{-4.4}^{+5.3 \mathrm{~b}}$ & $-0.034 \pm 0.01$ & $-0.18 \pm 0.01$ & & & & & \\
\hline PMI 13410+0542 & $51.3_{-11.8}^{+21.7 \mathrm{c}}$ & $0.047 \pm 0.008^{g}$ & $-0.014 \pm 0.008$ & 1.7 & 1.8 & 2.5 & 9.4 & 484 \\
\hline PMI 13410+0542 B & $44.3_{-7.2}^{+8.7}$ & $0.062 \pm 0.004$ & $-0.030 \pm 0.004$ & & & & & \\
\hline PMI 13518+4157 & $43.1_{-8.8}^{+9.9 \mathrm{~d}}$ & $-0.059 \pm 0.008^{\mathrm{g}}$ & $-0.058 \pm 0.008$ & 0.7 & 0.6 & 1.0 & 21.6 & 613 \\
\hline PMI 13518+4157 B & $39.6_{-6.6}^{+7.9}$ & $-0.05 \pm 0.009$ & $-0.065 \pm 0.009$ & & & & & \\
\hline NLTT 38489 & $62.3_{-17.0}^{+23.3 \mathrm{a}}$ & $-0.147 \pm 0.008^{f}$ & $0.183 \pm 0.008$ & 0.7 & 1.4 & 1.6 & 6.7 & 418 \\
\hline NLTT 38489 B & $128_{-21}^{+25 b}$ & $-0.141 \pm 0.004$ & $0.170 \pm 0.004$ & & & & & \\
\hline NLTT 39312 & $141_{-33}^{+43 a}$ & $-0.025 \pm 0.008^{f}$ & $-0.347 \pm 0.008$ & 4.3 & 1.2 & 4.4 & 5.1 & 713 \\
\hline NLTT 39312 B & $124_{-20}^{+24}$ & $0.09 \pm 0.02$ & $-0.32 \pm 0.02$ & & & & & \\
\hline LSPM J1627+3328 & $38.0_{-5.4}^{+6.3 \mathrm{c}}$ & $-0.171 \pm 0.008^{f}$ & $-0.014 \pm 0.008$ & 3.29 & -1.64 & 3.67 & 9.0 & 341 \\
\hline LSPM J1627+3328 & $49.9_{-8.1}^{+9.7}$ & $-0.140 \pm 0.005$ & $-0.030 \pm 0.005$ & & & & & \\
\hline NLTT 44368 & $55.0_{-11.3}^{+12.7 \mathrm{~d}}$ & $-0.056 \pm 0.008^{f}$ & $0.215 \pm 0.008$ & 0.33 & -0.37 & 0.49 & 90.2 & 7760 \\
\hline NLTT 44368 B & $35.3_{-5.8}^{+6.9 \mathrm{~b}}$ & $-0.053 \pm 0.006$ & $0.211 \pm 0.006$ & & & & & \\
\hline LSPM J1717+5925 & $108_{-26}^{+35 \mathrm{~d}}$ & $-0.085 \pm 0.008^{\mathrm{i}}$ & $-0.143 \pm 0.008$ & 1.8 & 1.6 & 2.4 & 14.4 & 1555 \\
\hline LSPM J1717+5925 B & $119_{-22}^{+27}$ & $-0.101 \pm 0.004$ & $-0.157 \pm 0.004$ & & & & & \\
\hline
\end{tabular}




\begin{tabular}{|c|c|c|c|c|c|c|c|c|}
\hline & & & $\begin{array}{c}\text { Table } 5 \\
\text { (Continued) }\end{array}$ & & & & & \\
\hline Object & $\begin{array}{c}\text { distance } \\
(\mathrm{pc})\end{array}$ & $\begin{array}{l}\mu_{\alpha} \cos \delta \\
\left({ }^{\prime \prime} \mathrm{yr}^{-1}\right)\end{array}$ & $\begin{array}{c}\mu_{\delta} \\
\left({ }^{\prime \prime} \mathrm{yr}^{-1}\right)\end{array}$ & $\begin{array}{c}d \mu_{\alpha} \cos \delta \\
n_{\sigma}\end{array}$ & $\begin{array}{c}d \mu_{\delta} \\
n_{\sigma}\end{array}$ & $\begin{array}{l}d \mu \\
n_{\sigma}\end{array}$ & $\begin{array}{l}\text { Projected } \\
\text { Separation } \\
\quad\left({ }^{\prime \prime}\right)\end{array}$ & $\begin{array}{c}\text { Projected } \\
\text { Separation } \\
(\mathrm{AU})\end{array}$ \\
\hline NLTT 52268 & $37.3_{-8.7}^{+11.3 \mathrm{a}}$ & $0.16 \pm 0.008$ & $-0.089 \pm 0.008^{f}$ & 0.5 & 0.2 & 0.6 & 14.7 & 549 \\
\hline NLTT 52268 B & $55.6_{-9.1}^{+10.8}$ & $0.155 \pm 0.006$ & $-0.087 \pm 0.006$ & & & & & \\
\hline LSPM J2153+1157 & $36.2_{-8.4}^{+10.9 \mathrm{a}}$ & $-0.093 \pm 0.008^{f}$ & $-0.128 \pm 0.008$ & 0.8 & 0.8 & 1.2 & 11.3 & 408 \\
\hline LSPM J2153+1157 B & $58.1_{-9.5}^{+11.4}$ & $-0.085 \pm 0.006$ & $-0.136 \pm 0.006$ & & & & & \\
\hline PM I22118-1005 & $37.4_{-7.7}^{+8.6 \mathrm{~d}}$ & $0.028 \pm 0.008^{\mathrm{g}}$ & $-0.25 \pm 0.008$ & 1.6 & 0.9 & 1.9 & 204.5 & 8892 \\
\hline PM I22118-1005 B & $43.9_{-7.3}^{+8.8}$ & $0.05 \pm 0.011$ & $-0.262 \pm 0.010$ & & & & & \\
\hline NLTT 55219 & $44.7_{-10.4}^{+13.5 \mathrm{a}}$ & $0.324 \pm 0.008^{\mathrm{f}}$ & $0.16 \pm 0.008$ & 0.9 & 3.1 & 3.2 & 9.7 & 432 \\
\hline NLTT 55219 B & $40.0_{-6.5}^{+7.8}$ & $0.35 \pm 0.03$ & $0.26 \pm 0.03$ & & & & & \\
\hline \multicolumn{9}{|c|}{ Serendipitous Companion Discoveries } \\
\hline NLTT 730 & $21.7_{-5.3}^{+8.1 \mathrm{~d}}$ & $0.375 \pm 0.008^{\mathrm{f}}$ & $-0.234 \pm 0.008$ & 0.5 & 1.58 & 1.66 & 233.6 & 5070 \\
\hline NLTT 730 B & $23.7_{-3.9}^{+4.6 \mathrm{~b}}$ & $0.381 \pm 0.009$ & $-0.215 \pm 0.009$ & & & & & \\
\hline NLTT 27966 & $39.6_{-10.2}^{+13.3 \mathrm{a}}$ & $-0.153 \pm 0.008^{\mathrm{f}}$ & $0.182 \pm 0.008$ & -0.79 & 0.42 & 0.89 & 15.9 & 630 \\
\hline NLTT 27966 B & $42.3_{-6.9}^{+8.2 b}$ & $-0.164 \pm 0.011$ & $0.188 \pm 0.011$ & & & & & \\
\hline LSPMJ1336+2541 & $60.7_{-13.9}^{+15.7 \mathrm{~d}}$ & $-0.157 \pm 0.008^{\mathrm{f}}$ & $0.057 \pm 0.008$ & 2.1 & 2.8 & 3.5 & 121.7 & 8793 \\
\hline LSPM J1336+2541 B & $63.2_{-10.3}^{+12.4 b}$ & $-0.177 \pm 0.005$ & $0.031 \pm 0.005$ & & & & & \\
\hline HIP 73169 & {$\left[27.3_{-6.3}^{+8.3 \mathrm{e}}\right]$} & $-0.267 \pm 0.007$ & $-0.076 \pm 0.007$ & 0.5 & 0.42 & 0.65 & 29.1 & 796 \\
\hline HIP 73169 B & $50.7_{-8.3}^{+9.9 \mathrm{~b}}$ & $-0.261 \pm 0.009$ & $-0.081 \pm 0.009$ & & & & & \\
\hline PM I23492+3458 & $30.7_{-6.3}^{+7.1 \mathrm{~d}}$ & $-0.011 \pm 0.008^{\mathrm{f}}$ & $-0.108 \pm 0.008$ & 1.0 & 1.0 & 1.4 & 34.9 & 949 \\
\hline PM I23492+3458 B & $23.3_{-3.8}^{+4.5 \mathrm{~b}}$ & $-0.002 \pm 0.005$ & $-0.099 \pm 0.005$ & & & & & \\
\hline \multicolumn{9}{|c|}{ Unlikely companions } \\
\hline NLTT 35593 & $63.0_{-14.6}^{+19.1 \mathrm{a}}$ & $-0.184 \pm 0.005^{\mathrm{h}}$ & $-0.055 \pm 0.005$ & 1.76 & -0.34 & 1.79 & 1105.8 & 69706 \\
\hline NLTT 35593 B & $65.5_{-10.7}^{+12.8 \mathrm{~b}}$ & $-0.162 \pm 0.011$ & $-0.059 \pm 0.011$ & & & & & \\
\hline
\end{tabular}

Notes. $n_{\sigma}$ is the number of standard deviations the proper motions differ by. [...] Denotes distance from trigonometric parallax; all other distances are photometric.

a Photometric distance from Lépine (2005).

b Photometric distance from 2MASS magnitudes and Dupuy \& Liu (2012).

${ }^{c}$ Photometric distance from Lépine \& Gaidos (2011).

${ }^{\mathrm{d}}$ Photometric distance from spectral type and spectrophotometric relations of Kraus \& Hillenbrand (2007).

e Trigonometric parallax from van Leeuwen (2007).

${ }^{\mathrm{f}}$ Proper motion from Lépine \& Shara (2005).

g Proper motion from Lépine \& Gaidos (2011).

${ }^{\text {h }}$ Proper motion from Salim \& Gould (2003).

${ }^{i}$ Proper motion from Hog et al. (2000).

\subsection{Optical Spectroscopy}

As a number of our primaries were poorly characterized, we obtained optical spectroscopy from both the Kitt Peak Mayall $4 \mathrm{~m}$ telescope and the University of Hawaii $2.2 \mathrm{~m}$ telescope.

\subsubsection{Kitt Peak Mayall 4 m Spectroscopy}

On 2013 December 31 UT we obtained optical spectra of NLTT 38389 and NLTT 1011 with the Ritchey-Chretien Spectrograph equipped with the T2KA CCD on the Kitt Peak National Observatory $4 \mathrm{~m}$ Mayall telescope. The BL 420 grating was blazed at $7800 \AA$ (first order) with the GG495 order blocking filter. The slit was set at 1 '.5 by $98^{\prime \prime}$, resulting in spectra spanning $6300-8500 \AA$ at a resolving power of $\sim 3 \AA$. NLTT 38389 and NLTT 1011 were targeted at airmasses of 1.25 and 1.01 with exposures of $600 \mathrm{~s}$ and $240 \mathrm{~s}$, respectively. We also acquired optical spectroscopy for one of our companions (HIP 84840 B) on 2014 May 22 UT with the same set-up at airmass 1.03. The slit was oriented in the $\mathrm{N}-\mathrm{S}$ direction for all observations, so slit losses from chromatic dispersion may be non-negligible for NLTT 38389. The spectrophotometric standards HR 3454 (Hamuy et al. 1992) (2013 December 31 UT) and HZ 44 (2014 May 22 UT) were observed at airmasses of 1.15 and 1.0, respectively, for flux calibration. Each 2D image was corrected for bad pixels and cosmic rays, bias subtracted, and flat fielded. After sky subtraction, each spectrum was corrected for throughput losses using our standard star measurements.

\subsubsection{University of Hawaii 2.2 m Telescope/SNIFS Spectroscopy}

To aid in characterizing the primary stars, we took spectra of GD 280, NLTT 730, LSPM J2153+1157, and NLTT 22073 with the SuperNova Integral Field Spectrograph (Aldering et al. 2002; Lantz 2004) on the University of Hawaii $2.2 \mathrm{~m}$ telescope on Mauna Kea on UT 2013 October 19 and December 13-14. 
Table 6

The IR Data of Our Secondaries

\begin{tabular}{|c|c|c|c|c|c|}
\hline Object & $\begin{array}{c}Y \\
(\mathrm{mag})\end{array}$ & $\begin{array}{c}J \\
(\mathrm{mag})\end{array}$ & $\begin{array}{c}H \\
(\mathrm{mag})\end{array}$ & $\begin{array}{l}K / K_{s} \\
(\mathrm{mag})\end{array}$ & Source \\
\hline \multicolumn{6}{|c|}{ HIP search companions } \\
\hline \multirow[t]{2}{*}{ HIP 2397 B } & $\ldots$ & $14.586 \pm 0.038^{*}$ & $13.975 \pm 0.048^{*}$ & $>13.181^{*}$ & 2MASS \\
\hline & $\ldots$ & $14.552 \pm 0.012$ & $13.912 \pm 0.011$ & & UKIRT \\
\hline \multirow[t]{2}{*}{ HIP 6217 B } & & $15.441 \pm 0.060^{*}$ & $14.731 \pm 0.081^{*}$ & $14.423 \pm 0.079^{*}$ & 2MASS \\
\hline & $\ldots$ & & & $14.461 \pm 0.009$ & UKIDSS \\
\hline \multirow[t]{2}{*}{ HIP 6407 B } & $\ldots$ & $15.471 \pm 0.046$ & $14.651 \pm 0.061$ & $14.402 \pm 0.08$ & 2MASS \\
\hline & $16.442 \pm 0.008$ & $15.345 \pm 0.005$ & $14.738 \pm 0.006$ & $14.203 \pm 0.006$ & UKIDSS \\
\hline \multirow[t]{2}{*}{ HIP 9269 B } & $\ldots$ & $15.956 \pm 0.088^{*}$ & $14.99 \pm 0.096^{*}$ & $14.317 \pm 0.085^{*}$ & 2MASS \\
\hline & $\ldots$ & $16.126 \pm 0.017$ & $15.082 \pm 0.014$ & $14.298 \pm 0.019$ & UKIRT \\
\hline \multirow[t]{2}{*}{ HIP 10033 B } & $\ldots$ & $13.761 \pm 0.027$ & $13.185 \pm 0.031$ & $12.929 \pm 0.033$ & 2MASS \\
\hline & $\ldots$ & $13.746 \pm 0.013$ & $13.245 \pm 0.011$ & $\ldots$ & UKIRT \\
\hline \multirow[t]{2}{*}{ HIP 11161 B } & $\ldots$ & $16.636 \pm 0.161^{*}$ & $15.77 \pm 0.173$ & $15.392 \pm 0.195^{*}$ & 2MASS \\
\hline & $\ldots$ & $16.512 \pm 0.019$ & $15.862 \pm 0.017$ & $15.25 \pm 0.028$ & UKIRT \\
\hline \multirow[t]{2}{*}{ HIP 13589 B } & $\ldots$ & $14.928 \pm 0.034$ & $14.275 \pm 0.037$ & $13.838 \pm 0.046$ & 2MASS \\
\hline & $\ldots$ & $14.794 \pm 0.034$ & $14.226 \pm 0.012$ & & UKIRT \\
\hline HIP 26653 B & & $14.756 \pm 0.044^{*}$ & $13.91 \pm 0.042^{*}$ & $13.322 \pm 0.029^{*}$ & 2MASS \\
\hline HIP 32728 B & $\ldots$ & $14.508 \pm 0.031$ & $13.933 \pm 0.038$ & $13.509 \pm 0.035$ & 2MASS \\
\hline HIP 37283 B & $\ldots$ & $12.797 \pm 0.027$ & $12.234 \pm 0.03$ & $11.943 \pm 0.024$ & 2MASS \\
\hline \multirow[t]{2}{*}{ HIP 46984 B } & $\ldots$ & $13.285 \pm 0.027^{*}$ & $12.745 \pm 0.024$ & $12.483 \pm 0.027^{*}$ & 2MASS \\
\hline & $13.794 \pm 0.002$ & $13.215 \pm 0.002$ & $12.767 \pm 0.002$ & $12.463 \pm 0.002$ & UKIDSS \\
\hline \multirow[t]{2}{*}{ HIP 49046 B } & $\ldots$ & $13.022 \pm 0.022$ & $12.418 \pm 0.024$ & $12.038 \pm 0.021$ & 2MASS \\
\hline & $\ldots$ & $12.930 \pm 0.092$ & $\ldots$ & $\ldots$ & UKIRT \\
\hline HIP 51877 B & & $15.205 \pm 0.063^{*}$ & $14.510 \pm 0.062^{*}$ & $13.988 \pm 0.052^{*}$ & 2MASS \\
\hline HIP 52463 B & $\ldots$ & $13.703 \pm 0.024$ & $13.117 \pm 0.027$ & $12.801 \pm 0.031$ & 2MASS \\
\hline \multirow[t]{2}{*}{ HIP 55666 B } & $\ldots$ & $12.674 \pm 0.024$ & $12.108 \pm 0.024$ & $11.746 \pm 0.019$ & 2MASS \\
\hline & $13.322 \pm 0.002$ & $12.581 \pm 0.001$ & $12.103 \pm 0.003$ & $11.762 \pm 0.002$ & VISTA \\
\hline HIP 58918 B & $\ldots$ & $14.471 \pm 0.03^{*}$ & $13.899 \pm 0.044$ & $13.524 \pm 0.023$ & 2MASS \\
\hline HIP 59310 B & $\ldots$ & $13.691 \pm 0.027$ & $13.052 \pm 0.033$ & $12.692 \pm 0.024$ & 2MASS \\
\hline \multirow[t]{2}{*}{ HIP 59933 B } & $\ldots$ & $16.101 \pm 0.088^{*}$ & $15.162 \pm 0.075^{*}$ & $14.616 \pm 0.084$ & 2MASS \\
\hline & $17.126 \pm 0.015$ & $15.995 \pm 0.008$ & $15.218 \pm 0.009$ & $14.62 \pm 0.01$ & UKIDSS \\
\hline \multirow[t]{2}{*}{ HIP 60501 B } & $\ldots$ & $11.378 \pm 0.027$ & $10.792 \pm 0.024$ & $10.453 \pm 0.023$ & 2MASS \\
\hline & $11.916 \pm 0.001$ & $11.309 \pm 0.001$ & $10.979 \pm 0.001$ & $10.554 \pm 0.001$ & UKIDSS \\
\hline HIP 60987 B & $\ldots$ & $\ldots$ & $\ldots$ & $10.869 \pm 0.001$ & VISTA $^{\dagger}$ \\
\hline HIP $60987 \mathrm{C}$ & $\ldots$ & $11.402 \pm 0.028^{*}$ & $10.81 \pm 0.029$ & $10.592 \pm 0.024$ & 2MASS \\
\hline & $11.782 \pm 0.001$ & $11.267 \pm 0.001$ & $10.7497 \pm 0.001$ & $10.488 \pm 0.001$ & VISTA \\
\hline HIP 63506 C & $\ldots$ & $15.15 \pm 0.042$ & $14.426 \pm 0.045$ & $14.006 \pm 0.047$ & 2MASS \\
\hline HIP 65706 B & $\ldots$ & $13.315 \pm 0.022$ & $12.642 \pm 0.026$ & $12.297 \pm 0.024$ & 2MASS \\
\hline & $14.011 \pm 0.002$ & $13.246 \pm 0.001$ & $12.693 \pm 0.001$ & $12.258 \pm 0.002$ & UKIDSS \\
\hline HIP $65780 \mathrm{C}$ & & $>12.717$ & $>12.197$ & $12.007 \pm 0.034^{*}$ & 2MASS \\
\hline & $13.580 \pm 0.002$ & $12.937 \pm 0.001$ & $12.404 \pm 0.002$ & $12.044 \pm 0.002$ & UKIDSS \\
\hline HIP $70623 \mathrm{C}$ & $\ldots$ & $12.191 \pm 0.026$ & $11.506 \pm 0.023$ & $11.224 \pm 0.019$ & 2MASS \\
\hline & $\ldots$ & $12.039 \pm 0.001$ & $11.541 \pm 0.001$ & $11.233 \pm 0.001$ & VISTA \\
\hline HIP 75310 B & $\ldots$ & $13.291 \pm 0.032^{*}$ & $12.724 \pm 0.03$ & $12.51 \pm 0.027$ & 2MASS \\
\hline HIP 76456 B & $\ldots$ & $13.300 \pm 0.031^{*}$ & $12.762 \pm 0.028^{*}$ & $12.43 \pm 0.029^{*}$ & 2MASS \\
\hline & $13.973 \pm 0.002$ & $13.240 \pm 0.002$ & $12.809 \pm 0.002$ & $12.397 \pm 0.002$ & UKIDSS \\
\hline HIP 76641 B & $\ldots$ & $10.817 \pm 0.018$ & $10.176 \pm 0.016$ & $9.919 \pm 0.014$ & 2MASS \\
\hline HIP 78184 B & $\ldots$ & $14.32 \pm 0.031$ & $13.607 \pm 0.035$ & $13.122 \pm 0.03$ & 2MASS \\
\hline & $\ldots$ & $14.252 \pm 0.022$ & $13.615 \pm 0.037$ & $13.091 \pm 0.028$ & UKIRT \\
\hline HIP 78859 B & $\ldots$ & $12.258 \pm 0.021$ & $11.694 \pm 0.02$ & $11.496 \pm 0.02$ & 2MASS \\
\hline & $\ldots$ & $12.214 \pm 0.010$ & $11.734 \pm 0.010$ & $\ldots$ & UKIRT \\
\hline HIP 78916 B & $\ldots$ & $15.227 \pm 0.043^{*}$ & $14.542 \pm 0.045$ & $14.193 \pm 0.053$ & 2MASS \\
\hline & $\ldots$ & $15.148 \pm 0.0179$ & $14.603 \pm 0.028$ & $\ldots$ & UKIRT \\
\hline HIP 78923 B & $\ldots$ & $14.052 \pm 0.048^{*}$ & $13.566 \pm 0.051^{*}$ & $13.28 \pm 0.041^{*}$ & 2MASS \\
\hline & $\cdots$ & $14.136 \pm 0.011$ & $13.621 \pm 0.11$ & 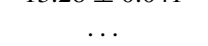 & UKIRT \\
\hline HIP 79180 B & $\ldots$ & $13.941 \pm 0.022$ & $13.361 \pm 0.027$ & $12.965 \pm 0.033$ & 2MASS \\
\hline HIP 80258 B & $\ldots$ & $13.113 \pm 0.021$ & $12.556 \pm 0.023$ & $12.28 \pm 0.021$ & 2MASS \\
\hline & $\ldots$ & $13.101 \pm 0.016$ & $12.562 \pm 0.010$ & $\ldots$ & UKIRT \\
\hline HIP 81910 B & $\ldots$ & $12.44 \pm 0.024^{*}$ & $11.887 \pm 0.026$ & $11.526 \pm 0.025$ & 2MASS \\
\hline & $\ldots$ & $12.400 \pm 0.010$ & $11.858 \pm 0.010$ & $\ldots$ & UKIRT \\
\hline HIP 82233 B & $\ldots$ & $11.889 \pm 0.024$ & $11.288 \pm 0.021$ & $11.043 \pm 0.023$ & 2MASS \\
\hline & $\ldots$ & $11.874 \pm 0.011$ & $11.366 \pm 0.010$ & $\ldots$ & UKIRT \\
\hline HIP 83651 B & $\ldots$ & $10.599 \pm 0.024$ & $10.057 \pm 0.024$ & $9.771 \pm 0.021$ & 2MASS \\
\hline & & $10.734 \pm 0.010$ & $10.877 \pm 0.011$ & & UKIRT \\
\hline HIP 84840 B & $\ldots$ & $12.508 \pm 0.022$ & $11.901 \pm 0.023$ & $11.685 \pm 0.018$ & 2MASS \\
\hline & $\ldots$ & $12.491 \pm 0.019$ & $11.950 \pm 0.010$ & $\ldots$ & UKIRT \\
\hline
\end{tabular}


Table 6

(Continued)

\begin{tabular}{|c|c|c|c|c|c|}
\hline Object & $\begin{array}{c}Y \\
(\mathrm{mag})\end{array}$ & $\begin{array}{c}J \\
(\mathrm{mag})\end{array}$ & $\begin{array}{c}H \\
(\mathrm{mag})\end{array}$ & $\begin{array}{l}K / K_{s} \\
(\mathrm{mag})\end{array}$ & Source \\
\hline \multirow[t]{2}{*}{ HIP 85365 B } & $\ldots$ & $16.693 \pm 0.168^{*}$ & $15.471 \pm 0.118$ & $>14.664$ & 2MASS \\
\hline & $\ldots$ & $16.184 \pm 0.010$ & $\ldots$ & $14.718 \pm 0.010$ & VISTA \\
\hline \multirow[t]{2}{*}{ HIP 86722 B } & $\ldots$ & $11.511 \pm 0.025$ & $11.016 \pm 0.021$ & $10.7 \pm 0.019$ & 2MASS \\
\hline & $\ldots$ & $11.506 \pm 0.015$ & $11.089 \pm 0.01$ & $\ldots$ & UKIRT \\
\hline \multirow[t]{2}{*}{ HIP 88728 B } & $\ldots$ & $10.269 \pm 0.097^{*}$ & $10.043 \pm 0.047^{*}$ & $9.876 \pm 0.057^{*}$ & 2MASS \\
\hline & $\ldots$ & $11.103 \pm 0.019$ & $10.965 \pm 0.018$ & $10.953 \pm 0.027$ & UKIRT \\
\hline \multirow[t]{2}{*}{ HIP 90273 B } & $\ldots$ & $11.56 \pm 0.022$ & $11.019 \pm 0.022$ & $10.731 \pm 0.018$ & 2MASS \\
\hline & $\ldots$ & $11.495 \pm 0.010$ & $11.012 \pm 0.018$ & & UKIRT \\
\hline \multirow[t]{2}{*}{ HIP 90869 B } & $\ldots$ & $11.101 \pm 0.029^{*}$ & $10.569 \pm 0.027$ & $10.358 \pm 0.023$ & 2MASS \\
\hline & $\ldots$ & $11.071 \pm 0.10$ & $10.818 \pm 0.010$ & 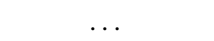 & UKIRT \\
\hline \multirow[t]{2}{*}{ HIP 93967 B } & $\ldots$ & $13.357 \pm 0.03$ & $12.74 \pm 0.025$ & $12.525 \pm 0.019$ & 2MASS \\
\hline & $\ldots$ & $13.24 \pm 0.02$ & $12.751 \pm 0.02$ & & UKIRT \\
\hline \multirow[t]{2}{*}{ HIP 97168 B } & $\ldots$ & $13.679 \pm 0.027^{*}$ & $13.212 \pm 0.026^{*}$ & $13.012 \pm 0.033^{*}$ & 2MASS \\
\hline & $\ldots$ & $13.784 \pm 0.014$ & $13.332 \pm 0.014$ & $\ldots$ & UKIRT \\
\hline \multirow[t]{2}{*}{ HIP 98535 C } & $\ldots$ & $13.252 \pm 0.031$ & $12.724 \pm 0.031$ & $12.445 \pm 0.032$ & 2MASS \\
\hline & $\ldots$ & $13.223 \pm 0.010$ & $12.752 \pm 0.010$ & & UKIRT \\
\hline \multirow[t]{2}{*}{ HIP 102582 B } & $\ldots$ & $11.963 \pm 0.033^{*}$ & $11.494 \pm 0.034$ & $11.232 \pm 0.032^{*}$ & 2MASS \\
\hline & $\ldots$ & $11.972 \pm 0.015$ & $11.551 \pm 0.010$ & & UKIRT \\
\hline \multirow[t]{2}{*}{ HIP 103199 B } & $\ldots$ & $11.817 \pm 0.027^{*}$ & $11.229 \pm 0.024^{*}$ & $10.997 \pm 0.019^{*}$ & 2MASS \\
\hline & $12.378 \pm 0.014$ & $11.850 \pm 0.18$ & $11.307 \pm 0.010$ & $11.250 \pm 0.010$ & UKIRT \\
\hline \multirow[t]{2}{*}{ HIP 105202 B } & $\ldots$ & $12.124 \pm 0.024$ & $11.53 \pm 0.022$ & $11.246 \pm 0.023$ & 2MASS \\
\hline & $\ldots$ & $12.0337 \pm 0.010$ & $11.571 \pm 0.014$ & & UKIRT \\
\hline \multirow[t]{2}{*}{ HIP 106551 B } & $\ldots$ & $13.224 \pm 0.025^{*}$ & $12.606 \pm 0.03$ & $12.287 \pm 0.022$ & 2MASS \\
\hline & $\ldots$ & $13.211 \pm 0.010$ & $12.630 \pm 0.010$ & 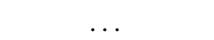 & UKIRT \\
\hline \multirow[t]{2}{*}{ HIP 108822 B } & $\ldots$ & $10.622 \pm 0.022$ & $10.051 \pm 0.021$ & $9.821 \pm 0.02$ & 2MASS \\
\hline & $\ldots$ & $10.805 \pm 0.020$ & $10.650 \pm 0.010$ & $\ldots$ & UKIRT \\
\hline \multirow[t]{2}{*}{ HIP 109454 B } & $\ldots$ & $12.422 \pm 0.026^{*}$ & $11.863 \pm 0.032$ & $11.62 \pm 0.02$ & 2MASS \\
\hline & $\ldots$ & $12.452 \pm 0.01$ & $11.904 \pm 0.017$ & & UKIRT \\
\hline HIP 111657 B & $\ldots$ & $10.587 \pm 0.032^{*}$ & $10.028 \pm 0.034^{*}$ & $9.73 \pm 0.027^{*}$ & 2MASS \\
\hline & $\ldots$ & $10.583 \pm 0.010$ & $10.545 \pm 0.016$ & & UKIRT \\
\hline HIP 112422 B & $\ldots$ & $16.023 \pm 0.113^{*}$ & $15.111 \pm 0.103^{*}$ & $14.651 \pm 0.093^{*}$ & 2MASS \\
\hline & $17.171 \pm 0.022$ & $16.016 \pm 0.017$ & $15.251 \pm 0.015$ & $\ldots$ & UKIRT \\
\hline HIP 114424 B & $\ldots$ & $11.595 \pm 0.026$ & $10.976 \pm 0.022$ & $10.663 \pm 0.022$ & 2MASS \\
\hline & $\ldots$ & $11.483 \pm 0.010$ & $11.185 \pm 0.010$ & & UKIRT \\
\hline HIP 114456 B & $\ldots$ & $10.844 \pm 0.021^{*}$ & $10.227 \pm 0.028^{*}$ & $9.896 \pm 0.024^{*}$ & 2MASS \\
\hline & $\ldots$ & $10.840 \pm 0.014$ & $10.446 \pm 0.010$ & & UKIRT \\
\hline HIP 115819 B & $\ldots$ & $15.095 \pm 0.034$ & $14.357 \pm 0.029$ & $13.974 \pm 0.045$ & 2MASS \\
\hline & $15.844 \pm 0.007$ & $14.965 \pm 0.005$ & $14.370 \pm 0.004$ & $13.907 \pm 0.005$ & UKIDSS \\
\hline HIP 116052 B & $\ldots$ & $12.361 \pm 0.019$ & $11.79 \pm 0.024$ & $11.512 \pm 0.025$ & 2MASS \\
\hline & $\ldots$ & $12.332 \pm 0.019$ & $11.793 \pm 0.010$ & $\ldots$ & UKIRT \\
\hline & & mpanions to faint no & P primaries & & \\
\hline NLTT 1011 & $\ldots$ & $15.544 \pm 0.059$ & $14.928 \pm 0.069$ & $14.318 \pm 0.066$ & 2MASS \\
\hline GD $280 \mathrm{~B}$ & & $16.034 \pm 0.082$ & $14.993 \pm 0.09$ & $14.547 \pm 0.08$ & 2MASS \\
\hline NLTT 8245 B & & $14.922 \pm 0.052^{*}$ & $14.221 \pm 0.068^{*}$ & $13.812 \pm 0.056^{*}$ & 2MASS \\
\hline LSPM J0241+2553 B & & $17.027 \pm 0.180$ & $16.028 \pm 0.180$ & $15.331 \pm 0.149$ & 2MASS \\
\hline HD 253662 B & & $15.961 \pm 0.082^{*}$ & $15.182 \pm 0.087^{*}$ & $14.506 \pm 0.095^{*}$ & 2MASS \\
\hline & $16.511 \pm 0.009$ & $15.653 \pm 0.007$ & $\ldots$ & & UKIDSS \\
\hline LSPM J0632+5053 B & $\ldots$ & $16.383 \pm 0.108$ & $15.421 \pm 0.133$ & $15.171 \pm 0.122$ & 2MASS \\
\hline & $\ldots$ & $16.612 \pm 0.027$ & $15.864 \pm 0.022$ & $\ldots$ & UKIRT \\
\hline NLTT 18587 B & & $16.894 \pm 0.199$ & $15.921 \pm 0.215$ & $>15.221$ & $2 \mathrm{MASS}^{\ddagger}$ \\
\hline NLTT 19109 B & & $16.933 \pm 0.17^{*}$ & $15.89 \pm 0.169^{*}$ & $15.463 \pm 0.21^{*}$ & 2MASS \\
\hline & $\ldots$ & $16.512 \pm 0.017$ & $\ldots$ & $15.679 \pm 0.026$ & VISTA \\
\hline NLTT 22073 B & $\ldots$ & $14.671 \pm 0.037$ & $14.070 \pm 0.044$ & $13.696 \pm 0.049$ & 2MASS \\
\hline NLTT 23716 B & & $14.300 \pm 0.036^{*}$ & $13.716 \pm 0.035^{*}$ & $13.348 \pm 0.028^{*}$ & 2MASS \\
\hline & $15.085 \pm 0.005$ & $14.225 \pm 0.003$ & $13.713 \pm 0.004$ & $13.309 \pm 0.005$ & VISTA \\
\hline NLTT 26746 B & & $16.403 \pm 0.118^{*}$ & $15.224 \pm 0.092$ & $14.555 \pm 0.105$ & 2MASS \\
\hline & $17.377 \pm 0.015$ & $16.056 \pm 0.008$ & $15.295 \pm 0.0090$ & $14.61 \pm 0.008$ & UKIDSS \\
\hline NLTT 29395 B & & $15.81 \pm 0.08^{*}$ & $15.133 \pm 0.076^{*}$ & $14.781 \pm 0.091^{*}$ & 2MASS \\
\hline NLTT $30510 \mathrm{~B}$ & & $15.971 \pm 0.08^{*}$ & $15.267 \pm 0.095$ & $14.854 \pm 0.09$ & 2MASS \\
\hline NLTT $31450 \mathrm{~B}$ & $\ldots$ & $16.135 \pm 0.105^{*}$ & $15.048 \pm 0.095^{*}$ & $14.331 \pm 0.092^{*}$ & 2MASS \\
\hline & $\ldots$ & $16.004 \pm 0.21$ & $15.081 \pm 0.023$ & $\ldots$ & UKIRT \\
\hline PMI 13410+0542 B & $\ldots$ & $16.187 \pm 0.143^{*}$ & $15.128 \pm 0.131^{*}$ & $14.799 \pm 0.138^{*}$ & 2MASS \\
\hline & $17.349 \pm 0.017$ & $16.125 \pm 0.009$ & $15.358 \pm 0.008$ & $14.727 \pm 0.008$ & UKIDSS \\
\hline PMI 13518+4157 B & $\ldots$ & $15.081 \pm 0.032$ & $14.352 \pm 0.049$ & $13.851 \pm 0.04$ & 2MASS \\
\hline
\end{tabular}




\begin{tabular}{|c|c|c|c|c|c|}
\hline \multicolumn{6}{|c|}{$\begin{array}{c}\text { Table } 6 \\
\text { (Continued) }\end{array}$} \\
\hline Object & $\begin{array}{c}Y \\
(\mathrm{mag})\end{array}$ & $\begin{array}{c}J \\
(\mathrm{mag})\end{array}$ & $\begin{array}{c}H \\
(\mathrm{mag})\end{array}$ & $\begin{array}{l}K / K_{s} \\
(\mathrm{mag})\end{array}$ & Source \\
\hline \multirow[t]{2}{*}{ NLTT 38489 B } & $\ldots$ & $16.64 \pm 0.143^{*}$ & $15.789 \pm 0.176^{*}$ & $15.481 \pm 0.154^{*}$ & 2MASS \\
\hline & $\ldots$ & $16.717 \pm 0.031$ & $16.147 \pm 0.037$ & & UKIRT \\
\hline \multirow[t]{2}{*}{ NLTT 39312 B } & $\ldots$ & $16.09 \pm 0.096^{*}$ & $15.573 \pm 0.118^{*}$ & $>13.601$ & 2MASS \\
\hline & $\ldots$ & $16.277 \pm 0.035$ & $16.006 \pm 0.045$ & $\ldots$ & UKIRT \\
\hline \multirow[t]{2}{*}{ LSPM J1627+3328 B } & $\ldots$ & $14.362 \pm 0.083^{*}$ & $13.742 \pm 0.066^{*}$ & $13.417 \pm 0.055^{*}$ & 2MASS \\
\hline & $\ldots$ & $14.730 \pm 0.012$ & $14.052 \pm 0.011$ & & UKIRT \\
\hline \multirow[t]{2}{*}{ NLTT 44368 B } & $\ldots$ & $14.736 \pm 0.058$ & $13.907 \pm 0.064$ & $13.467 \pm 0.049^{*}$ & 2MASS \\
\hline & $\ldots$ & $14.637 \pm 0.013$ & $13.993 \pm 0.012$ & & UKIRT \\
\hline \multirow[t]{2}{*}{ LSPM J1717+5925 B } & $\ldots$ & $16.580 \pm 0.168^{*}$ & $15.934 \pm 0.174^{*}$ & $15.581 \pm 0.255^{*}$ & 2MASS \\
\hline & $\ldots$ & $16.509 \pm 0.018$ & $\ldots$ & $\ldots$ & UHS \\
\hline \multirow[t]{2}{*}{ NLTT 52268 B } & $\ldots$ & $14.945 \pm 0.052^{*}$ & $14.29 \pm 0.05$ & $13.839 \pm 0.05$ & 2MASS \\
\hline & $\ldots$ & $14.950 \pm 0.02$ & $14.295 \pm 0.015$ & $\ldots$ & UKIRT \\
\hline \multirow[t]{2}{*}{ LSPM J2153+1157 B } & $\ldots$ & $14.403 \pm 0.038^{*}$ & $13.785 \pm 0.044^{*}$ & $13.427 \pm 0.045^{*}$ & 2MASS \\
\hline & $\ldots$ & $14.450 \pm 0.011$ & $13.834 \pm 0.011$ & $13.368 \pm 0.011$ & UKIRT \\
\hline PM I22118-1005 B & $\ldots$ & $15.246 \pm 0.049$ & $14.69 \pm 0.063$ & $14.051 \pm 0.052$ & 2MASS \\
\hline \multirow[t]{2}{*}{ NLTT 55219 B } & $\ldots$ & $16.313 \pm 0.122^{*}$ & $15.469 \pm 0.137^{*}$ & $14.778 \pm 0.145^{*}$ & 2MASS \\
\hline & $\ldots$ & $16.612 \pm 0.028$ & $15.769 \pm 0.024$ & $\ldots$ & UKIRT \\
\hline \multicolumn{6}{|c|}{ Serendipitous Companion Discoveries } \\
\hline \multirow[t]{2}{*}{ NLTT $730 \mathrm{~B}$} & $\ldots$ & $16.16 \pm 0.08$ & $15.23 \pm 0.08$ & $14.48 \pm 0.07$ & 2MASS \\
\hline & $\ldots$ & $16.16 \pm 0.03$ & $15.22 \pm 0.03$ & $\ldots$ & UKIRT \\
\hline \multirow[t]{2}{*}{ NLTT 27966 B } & $\ldots$ & $16.155 \pm 0.103$ & $15.27 \pm 0.11$ & $14.58 \pm 0.082$ & 2MASS \\
\hline & $17.308 \pm 0.044$ & $16.106 \pm 0.019$ & $15.283 \pm 0.018$ & $\ldots$ & UKIRT \\
\hline \multirow[t]{2}{*}{ LSPM J1336+2541 B } & $\ldots$ & $16.863 \pm 0.16$ & $16.017 \pm 0.172$ & $15.713 \pm 0.2$ & 2MASS \\
\hline & $18.228 \pm 0.033$ & $16.935 \pm 0.017$ & $16.122 \pm 0.018$ & $15.478 \pm 0.016$ & UKIDSS \\
\hline \multirow[t]{2}{*}{ HIP 73169 B } & & $16.007 \pm 0.09^{*}$ & $15.047 \pm 0.083$ & $14.36 \pm 0.081$ & 2MASS \\
\hline & $17.223 \pm 0.033$ & $15.929 \pm 0.02$ & $15.054 \pm 0.015$ & $14.446 \pm 0.016$ & VISTA \\
\hline \multirow[t]{2}{*}{ PM I23492+3458 B } & $\ldots$ & $16.298 \pm 0.105$ & $15.45 \pm 0.112$ & $15.008 \pm 0.127$ & 2MASS \\
\hline & $\ldots$ & $16.387 \pm 0.029$ & $15.472 \pm 0.021$ & $\ldots$ & UKIRT \\
\hline \multicolumn{6}{|c|}{ Unlikely companions } \\
\hline \multirow[t]{2}{*}{ NLTT 35593 B } & & $16.299 \pm 0.114$ & $15.408 \pm 0.117$ & $14.737 \pm 0.098$ & 2MASS \\
\hline & $17.468 \pm 0.02$ & $16.227 \pm 0.01$ & $15.469 \pm 0.011$ & $14.876 \pm 0.011$ & VISTA \\
\hline
\end{tabular}

Notes.

$\dagger$ Object saturated in VISTA data.

* Object has a confusion flag (ccflag) set in this filter.

${ }^{\ddagger}$ Photometry from the 2MASS reject table.

SNIFS provides simultaneous coverage from $3200 \AA$ to $9700 \AA$ at a resolution of $R \sim 1000$. Integration times varied from 65 to $500 \mathrm{~s}$, depending on the $R$ magnitude. This was sufficient to get reasonable $\mathrm{S} / \mathrm{N}$ ( $>70$ at $6000 \AA$ ) on all targets except GD 280, which had particularly low S/N $(\sim 15)$ due to patchy cloud cover and a fainter magnitude. The SNIFS pipeline (Bacon et al. 2001) performed basic reduction, including dark, bias, and flat-field corrections, wavelength calibration, sky subtraction, and extraction of the 1D spectrum. We took spectra of the spectrophotometric standards EG 131, Fiege 110, GD 71, Feige 66, and HR 7596 during the night, which were then used to flux calibrate the data and remove telluric lines. Additional information on SNIFS data processing can be found in Lépine et al. (2013) and Mann et al. (2013b).

\section{NEW COMMON PROPER MOTION COMPANIONS}

In total we have spectrally typed 87 companions to nearby stars of which 56 are new discoveries. Of these, 24 of our new discoveries are L dwarf companions, 1 is a late $\mathrm{K}, 2$ are DA white dwarfs, 23 M7-M9.5 dwarfs, with the remaining 39 being M0-M6.5 dwarfs. Finder charts for our new companions are shown in Figure 7. In addition, we identified one previously known blue L dwarf (2MASS J00150206+2959323, Kirkpatrick et al. 2010) as a companion to an M dwarf (NLTT 730), and a candidate L dwarf companion to an M dwarf (NLTT 35593), which we consider an unlikely companion to its $\mathrm{M}$ dwarf primary.

\subsection{Companionship Checks}

In order to assess whether our candidate companions are merely alignments of unassociated stars, we undertook a test similar to Lépine \& Bongiorno (2007). This consisted of taking all the objects in our input lists (Hipparcos, LSPM/rNLTT, and Lépine \& Gaidos 2011) and offsetting their positions by two degrees in right ascension. We then searched for common proper motion companions to this list of modified positions. This test should only yield non-physical (coincident) pairings. To accurately reflect the probability of our objects being coincident contaminants, we applied the same cuts that were applied to our initial target sample. As an additional cut to exclude spuriously red objects (which we have excluded from our candidate sample by checking the objects' $g_{P 1}, r_{P 1}$, and $i_{P 1}$ magnitudes) we excluded objects with more than one $g_{P 1}$ detection.

In Figure 8, we compare the separation and proper motion differences for our HIP samples and the coincident population. 
Table 7

The WISE Data of Our Companions

\begin{tabular}{|c|c|c|c|c|c|}
\hline Object & $\begin{array}{c}W 1 \\
(\mathrm{mag})\end{array}$ & $\begin{array}{c}W 2 \\
(\mathrm{mag})\end{array}$ & $\begin{array}{c}W 3 \\
\text { (mag) }\end{array}$ & $\begin{array}{c}W 4 \\
(\mathrm{mag})\end{array}$ & Note \\
\hline \multicolumn{6}{|c|}{ HIP search companions } \\
\hline HIP 2397 B & $13.077 \pm 0.026$ & $12.818 \pm 0.027$ & 12.167 & $8.924 \pm 0.409$ & \\
\hline HIP 6217 B & $14.154 \pm 0.03^{*}$ & $13.836 \pm 0.042^{*}$ & $>12.790$ & $>8.943$ & \\
\hline HIP 6407 B & $13.91 \pm 0.027^{*}$ & $13.584 \pm 0.035^{*}$ & $>12.375$ & $>9.305$ & \\
\hline HIP 9269 B & $13.486 \pm 0.026^{*}$ & $13.265 \pm 0.033^{*}$ & $>12.573^{*}$ & $9.119 \pm 0.473$ & \\
\hline HIP 10033 B & $12.597 \pm 0.023^{*}$ & $12.33 \pm 0.023^{*}$ & $12.578 \pm 0.337^{*}$ & $>9.229$ & \\
\hline HIP $11161 \mathrm{~B}$ & $15.173 \pm 0.043^{*}$ & $15.274 \pm 0.111^{*}$ & $>12.738^{*}$ & $>9.179$ & \\
\hline HIP 13589 B & $13.572 \pm 0.026^{*}$ & $13.295 \pm 0.035^{*}$ & $>12.558^{*}$ & $>9.002$ & \\
\hline HIP 26653 B & $\ldots$ & $\ldots$ & $\ldots$ & $\ldots$ & \\
\hline HIP 32728 B & $13.326 \pm 0027$ & $13.133 \pm 0.033$ & $>12.04$ & $>9.08$ & \\
\hline HIP 37283 B & $11.631 \pm 0.024^{*}$ & $11.425 \pm 0.021^{*}$ & $11.101 \pm 0.105$ & 9.329 & \\
\hline HIP 46984 B & $12.313 \pm 0.025^{*}$ & $12.129 \pm 0.024^{*}$ & $12.3 \pm 0.4$ & $>8.6$ & \\
\hline HIP 49046 B & $11.777 \pm 0.025$ & $11.582 \pm 0.023$ & $11.424 \pm 0.145$ & 8.916 & \\
\hline \multicolumn{6}{|l|}{ HIP 51877 B } \\
\hline HIP 52463 B & $12.595 \pm 0.025^{*}$ & $12.44 \pm 0.025^{*}$ & $12.1 \pm 0.3$ & $>8.9$ & \\
\hline HIP 55666 B & $11.525 \pm 0.023^{*}$ & $11.289 \pm 0.023^{*}$ & $11.463 \pm 0.176$ & 9.097 & \\
\hline HIP 58918 B & $13.252 \pm 0.025^{*}$ & $12.954 \pm 0.029^{*}$ & $>12.284$ & $>9.101$ & \\
\hline HIP 59310 B & $12.400 \pm 0.024$ & $12.198 \pm 0.024^{*}$ & $12.232 \pm 0.310$ & $>8.855$ & \\
\hline HIP 59933 B & $14.271 \pm 0.031^{*}$ & $13.939 \pm 0.048^{*}$ & $>12.707$ & $>8.876$ & \\
\hline HIP 60501 B & $10.281 \pm 0.023$ & $10.12 \pm 0.022^{*}$ & $10.038 \pm 0.067$ & $8.39 \pm 0.325$ & \\
\hline HIP 60987 B & $\ldots$ & $\ldots$ & $\ldots$ & \multirow[t]{2}{*}{$\ldots$} & \multirow[t]{4}{*}{$\dagger$} \\
\hline HIP $60987 \mathrm{C}$ & $10.434 \pm 0.022^{*}$ & $10.279 \pm 0.019^{*}$ & $10.167 \pm 0.066>8.887$ & & \\
\hline HIP $63506 \mathrm{C}$ & $13.696 \pm 0.027$ & $13.405 \pm 0.031$ & $>12.498$ & $>9.324$ & \\
\hline HIP 65706 B & $12.007 \pm 0.024^{*}$ & $11.799 \pm 0.023^{*}$ & $11.697 \pm 0.162$ & $>9.243$ & \\
\hline HIP $65780 \mathrm{C}$ & $\ldots$ & $\ldots$ & $\ldots$ & $\ldots$ & $\dagger$ \\
\hline HIP $70623 \mathrm{C}$ & $11.06 \pm 0.022^{*}$ & $10.921 \pm 0.021^{*}$ & $10.851 \pm 0.084$ & $>8.628$ & \\
\hline HIP 75310 B & $12.181 \pm 0.025^{*}$ & $11.984 \pm 0.024^{*}$ & $11.342 \pm 0.178$ & $>8.493$ & \\
\hline HIP 76456 B & $12.139 \pm 0.023^{*}$ & $11.867 \pm 0.022^{*}$ & $11.525 \pm 0.100^{*}$ & $>8.974$ & \\
\hline HIP 76641 B & $9.776 \pm 0.023^{*}$ & $9.676 \pm 0.021^{*}$ & 9.5590 .024 & $9.362 \pm 0.360$ & \\
\hline HIP 78184 B & $12.8 \pm 0.023$ & $12.516 \pm 0.022$ & $12.403 \pm 0.153$ & $>9.755$ & \\
\hline HIP $78859 \mathrm{~B}$ & $11.285 \pm 0.022^{*}$ & $11.086 \pm 0.021^{*}$ & $11.038 \pm 0.059$ & $>9.703$ & \\
\hline HIP 78916 B & $\ldots$ & $\ldots$ & $\ldots$ & $\ldots$ & $\dagger$ \\
\hline HIP 78923 B & $\ldots$ & $\ldots$ & $\ldots$ & $\ldots$ & $\dagger$ \\
\hline HIP 79180 B & $12.746 \pm 0.023^{*}$ & $12.558 \pm 0.022^{*}$ & $12.241 \pm 0.157$ & $>9.305$ & \\
\hline HIP 80258 B & $12.078 \pm 0.024$ & $11.884 \pm 0.024^{*}$ & $12.218 \pm 0.248$ & $>9.435$ & \\
\hline HIP 81910 B & $11.143 \pm 0.025^{*}$ & $11.011 \pm 0.025^{*}$ & $10.793 \pm 0.147^{*}$ & $>8.691$ & \\
\hline HIP 82233 B & $10.855 \pm 0.023^{*}$ & $10.661 \pm 0.02^{*}$ & $10.588 \pm 0.092$ & $>8.774$ & \\
\hline HIP 83651 B & $9.631 \pm 0.024^{*}$ & $9.495 \pm 0.021^{*}$ & $9.387 \pm 0.033$ & $9.004 \pm 0.428$ & \\
\hline HIP 84840 B & $11.45 \pm 0.023^{*}$ & $11.294 \pm 0.021^{*}$ & $11.376 \pm 0.105^{*}$ & $>9.348$ & \\
\hline HIP 85365 B & $13.875 \pm 0.03$ & $13.643 \pm 0.044$ & $>12.394$ & $>9.059$ & \\
\hline HIP 86722 B & $10.517 \pm 0.024^{*}$ & $10.253 \pm 0.022^{*}$ & $9.908 \pm 0.041^{*}$ & $9.376 \pm 0.54$ & \\
\hline HIP 88728 B & $\ldots$ & $\ldots$ & $\ldots$ & $\ldots$ & \\
\hline HIP 90273 B & $10.523 \pm 0.023^{*}$ & $10.361 \pm 0.021^{*}$ & $10.208 \pm 0.048$ & $9.386 \pm 0.513$ & \\
\hline HIP $90869 \mathrm{~B}$ & $10.148 \pm 0.022^{*}$ & $10.012 \pm 0.020^{*}$ & $10.089 \pm 0.064$ & $8.217 \pm 0.288$ & \\
\hline HIP 93967 B & $12.331 \pm 0.023^{*}$ & $12.137 \pm 0.026$ & $>11.605$ & $>8.52$ & \\
\hline HIP 97168 B & $\ldots$ & $\ldots$ & $\ldots$ & $\ldots$ & $\dagger$ \\
\hline HIP 98535 C & $12.14 \pm 0.023^{*}$ & $11.917 \pm 0.021^{*}$ & $12.603 \pm 0.321$ & $>9.516$ & \\
\hline HIP 102582 B & $11.059 \pm 0.025^{*}$ & $10.795 \pm 0.026^{*}$ & $10.771 \pm 0.096$ & $>9.105$ & \\
\hline HIP 103199 B & $10.855 \pm 0.056^{*}$ & $10.644 \pm 0.041^{*}$ & $10.61 \pm 0.107$ & $>9.209$ & \\
\hline HIP 105202 B & $11.046 \pm 0.024^{*}$ & $10.865 \pm 0.021^{*}$ & $10.583 \pm 0.08$ & $>9.01$ & \\
\hline HIP $106551 \mathrm{~B}$ & $\ldots$ & & $\ldots$ & $\ldots$ & $\dagger$ \\
\hline HIP 108822 B & $9.708 \pm 0.024^{*}$ & $9.564 \pm 0.020^{*}$ & $9.461 \pm 0.032$ & $8.63 \pm 0.276$ & \\
\hline HIP 109454 B & $11.5 \pm 0.025^{*}$ & $11.233 \pm 0.022^{*}$ & $11.171 \pm 0.111$ & $>9.279$ & \\
\hline HIP 111657 B & $9.566 \pm 0.050^{*}$ & $9.371 \pm 0.038^{*}$ & $9.259 \pm 0.05$ & $8.819 \pm 0.517$ & \\
\hline HIP 112422 B & $\ldots$ & 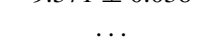 & $\ldots$ & $\ldots$ & $\dagger$ \\
\hline HIP 114424 B & $10.418 \pm 0.023^{*}$ & $10.213 \pm 0.020^{*}$ & $10.154 \pm 0.062$ & $>8.394$ & \\
\hline HIP 114456 B & $9.685 \pm 0.023^{*}$ & $9.511 \pm 0.020^{*}$ & $9.299 \pm 0.025^{*}$ & $8.744 \pm 0.242$ & \\
\hline HIP 115819 B & $13.511 \pm 0.026^{*}$ & $13.184 \pm 0.033^{*}$ & $12.42 \pm 0.467$ & $8.425 \pm 0.313$ & \\
\hline HIP 116052 B & $11.324 \pm 0.025^{*}$ & $11.135 \pm 0.023^{*}$ & $10.925 \pm 0.091$ & $>9.236$ & \\
\hline \multicolumn{6}{|c|}{ Companions to faint non-HIP primaries } \\
\hline NLTT 1011 & $13.762 \pm 0.026$ & $13.414 \pm 0.032^{*}$ & $>12.3$ & $>8.7$ & \\
\hline GD $280 \mathrm{~B}$ & $14.396 \pm 0.032$ & $14.149 \pm 0.052$ & $>12.794$ & $>9.206$ & \\
\hline NLTT 8245 B & $\ldots$ & $\ldots$ & $\ldots$ & $\ldots$ & $\dagger$ \\
\hline LSPM J0241+2553 B & $14.994 \pm 0.044$ & $14.826 \pm 0.093$ & $12.301 \pm 0.396$ & $>8.97$ & \\
\hline
\end{tabular}


Table 7

(Continued)

\begin{tabular}{|c|c|c|c|c|c|}
\hline Object & $\begin{array}{c}W 1 \\
(\mathrm{mag})\end{array}$ & $\begin{array}{c}W 2 \\
(\mathrm{mag})\end{array}$ & $\begin{array}{c}W 3 \\
\text { (mag) }\end{array}$ & $\begin{array}{c}W 4 \\
(\mathrm{mag})\end{array}$ & Note \\
\hline \multicolumn{6}{|l|}{ HD 253662 B } \\
\hline LSPM J0632+5053 B & $14.94 \pm 0.038^{*}$ & $14.757 \pm 0.078$ & $>12.814$ & $>9.183$ & \\
\hline NLTT 18587 B & $15.096 \pm 0.043$ & $14.828 \pm 0.085$ & $>12.008$ & $>9.129$ & \\
\hline NLTT 19109 B & $\ldots$ & $\ldots$ & $\ldots$ & $\ldots$ & $\dagger$ \\
\hline NLTT 22073 B & $13.295 \pm 0.027^{*}$ & $13.000 \pm 0.030^{*}$ & $>12.224$ & $>8.756$ & \\
\hline NLTT $23716 \mathrm{~B}$ & $\ldots$ & $\ldots$ & $\ldots$ & $\ldots$ & $\dagger$ \\
\hline NLTT 26746 B & $14.058 \pm 0.029^{*}$ & $13.871 \pm 0.045$ & $>12.715$ & $>8.755$ & \\
\hline NLTT 29395 B & $\ldots$ & $\ldots$ & $\ldots$ & $\ldots$ & $\dagger$ \\
\hline NLTT $30510 \mathrm{~B}$ & $14.546 \pm 0.032^{*}$ & $14.247 \pm 0.051^{*}$ & $>12.617$ & $>8.982$ & \\
\hline NLTT 31450 B & $\ldots$ & $\ldots$ & $\ldots$ & $\ldots$ & $\dagger$ \\
\hline PM I13410+0542 & $\ldots$ & $\ldots$ & $\ldots$ & $\ldots$ & $\dagger$ \\
\hline PM I13518+4157 & $13.556 \pm 0.026^{*}$ & $13.222 \pm 0.029$ & $12.46 \pm 0.31$ & $>9.328$ & \\
\hline NLTT 38489 B & $\ldots$ & $\ldots$ & $\ldots$ & $\ldots$ & $\dagger$ \\
\hline NLTT 39312 B & $\ldots$ & $\ldots$ & $\ldots$ & $\ldots$ & $\dagger$ \\
\hline LSPM J1627+3328 B & $\ldots$ & $\ldots$ & $\ldots$ & $\ldots$ & $\dagger$ \\
\hline NLTT 44368 B & $12.951 \pm 0.026$ & $12.692 \pm 0.025$ & $12.054 \pm 0.15$ & $>8.934$ & \\
\hline LSPM J1717+5925 B & $\ldots$ & $\ldots$ & $\ldots$ & $\ldots$ & $\dagger$ \\
\hline NLTT 52268 B & $\ldots$ & $\ldots$ & $\ldots$ & $\ldots$ & $\dagger$ \\
\hline LSPM J2153+1157 B & $\ldots$ & $\ldots$ & $\ldots$ & $\ldots$ & $\dagger$ \\
\hline PM I22118-1005 B & $13.571 \pm 0.027$ & $13.335 \pm 0.036$ & $>11.969$ & $>8.984$ & \\
\hline NLTT $55219 \mathrm{~B}$ & $\ldots$ & $\ldots$ & $\ldots$ & $\ldots$ & $\dagger$ \\
\hline \multicolumn{6}{|c|}{ Serendipitous Companion Discoveries } \\
\hline NLTT 730 B & $13.69 \pm 0.03$ & $13.38 \pm 0.04$ & $11.7 \pm 0.2$ & $>9.011$ & \\
\hline NLTT 27966 B & $14.204 \pm 0.028$ & $13.853 \pm 0.042$ & $>12.773$ & $>9.18$ & \\
\hline LSPM J1336+2541 B & $14.844 \pm 0.034$ & $14.553 \pm 0.06$ & $>12.869$ & $>8.916$ & \\
\hline HIP $73169 \mathrm{~B}$ & $13.988 \pm 0.027^{*}$ & $13.678 \pm 0.035^{*}$ & $12.692 \pm 0.409$ & $>9.423$ & \\
\hline PM I23492+3458 B & $14.036 \pm 0.028^{*}$ & $13.396 \pm 0.032^{*}$ & $12.311 \pm 0.305$ & $>8.985$ & \\
\hline \multicolumn{6}{|c|}{ Unlikely companions } \\
\hline NLTT 35593 B & $14.44 \pm 0.03$ & $14.287 \pm 0.052$ & $>12.517$ & $>9.277$ & \\
\hline
\end{tabular}

Notes.

* Has contaminated photometry in at least one WISE band.

${ }^{\dagger}$ Too close to their primary to have an independent photometry measurement in WISE.

All objects in both our bright and ultracool companion samples that were followed up spectroscopically lie in portions of the plot sparsely populated by coincident pairings. This is likely due to our approach of preferentially following-up closer companions. Figure 9 shows the results for companions to nonHipparcos primaries and our serendipitous discoveries. Most of our discoveries lie in a completely different area of the plot from the coincident pairings. However, one object, the apparent companion to NLTT 35593, is in a region of the diagram populated by many coincident pairings, and therefore we do not consider it to be a bona-fide companion. Excluding this object, we consider it likely that less than five of our companions to Hipparcos stars will be chance alignments with background stars. For our faint non-Hipparcos primaries, at most, two could be chance alignments. All of these coincident pairs would lie in the lowest proper motion bin of our sample.

\subsubsection{Photometric Distance}

To further check the companionship of the objects, we derived photometric distances for all of our companions based on their spectral type. For objects of spectral types M6 and later, we derived the absolute magnitudes for our objects using the relations of Dupuy \& Liu (2012) in $J, H$, and $K / K_{S}$. For earlier-type objects, we used the spectral energy distribution
(SED) templates of Kraus \& Hillenbrand (2007), who do not quote an rms for their relations. To estimate this we calculated the standard deviation of non-saturated 2MASS photometry of the FGK stars in Valenti \& Fischer (2005) about the main sequence. These were found to be $0.25,0.22$, and $0.22 \mathrm{mag}$ in $J, H$ and $K_{s}$, respectively. Cruz \& Reid (2002) calculated fits to the $J$-band absolute magnitude as a function of temperaturesensitive spectral features for $M$ dwarfs. They found that the M dwarf population was well-fitted by two relations, one covering early $\mathrm{M}$ with an rms of $\sim 0.2 \mathrm{mag}$ and the other covering late $\mathrm{M}$ with an rms of $\sim 0.35 \mathrm{mag}$. This latter number is comparable with the 0.39 mag rms on the 2MASS $J$-band relation of Dupuy \& Liu (2012). Hence we use the rms of Dupuy \& Liu (2012) for objects of spectral type M5 and later and our calculated rms for earlier-type objects.

Next, we calculated absolutes magnitude from the Kraus \& Hillenbrand (2007) or Dupuy \& Liu (2012) relations and compared these with the object's 2MASS photometry, or if available VISTA, UKIDSS, or UKIRT photometry, producing a distance estimate for each filter. Where non-2MASS nearinfrared photometry was available for an object in a particular filter, we used this instead of 2MASS, making use of the Dupuy \& Liu (2012) MKO relations for ultracool dwarfs and converting to the 2MASS system using the transformations of Carpenter (2001) for comparisons to the Kraus \& Hillenbrand 
Table 8

Log of Spectroscopic Observations

\begin{tabular}{|c|c|c|c|c|c|c|c|c|c|}
\hline Object & $\begin{array}{l}\text { Date } \\
\text { (UT) }\end{array}$ & $\begin{array}{l}t_{\text {int }} \\
(\mathrm{s})\end{array}$ & Conditions & $\begin{array}{c}\text { Seeing } \\
\left({ }^{\prime \prime}\right)\end{array}$ & $\begin{array}{l}\text { Slit } \\
\left({ }^{\prime \prime}\right)\end{array}$ & Mode & $\begin{array}{c}\text { Resolution } \\
\lambda / \Delta \lambda\end{array}$ & Airmass & Standard \\
\hline \multicolumn{10}{|c|}{ HIP search companions } \\
\hline HIP 2397 B & 2012 Jul 5 & 360 & cloudy & 1.5 & $0.8 \times 15$ & prism & 75 & 1.12 & HD 222749 \\
\hline HIP 6217 B & 2013 Dec 11 & 360 & cloudy & 1.3 & $0.5 \times 15$ & prism & 120 & 1.30 & HD 13936 \\
\hline HIP 6407 B & 2012 Jul 6 & 480 & clear & 1.3 & $0.3 \times 15$ & prism & 200 & 1.37 & HD 219545 \\
\hline HIP 9269 B & 2011 Dec 2 & 1440 & thin clouds & 0.4 & $0.8 \times 15$ & prism & 75 & 1.156 & HD 23452 \\
\hline HIP 10033 B & 2012 Oct 25 & 720 & cloudy & 0.5 & $0.8 \times 15$ & prism & 75 & 1.14 & HD 13936 \\
\hline HIP $11161 \mathrm{~B}$ & 2012 Oct 28 & 720 & thin clouds & 0.8 & $0.8 \times 15$ & prism & 75 & 1.202 & HD 21038 \\
\hline HIP 13589 B & 2012 Jul 8 & 480 & clear & $>1$ & $0.8 \times 15$ & prism & 75 & 1.44 & HD 19600 \\
\hline HIP 26653 B & 2014 Jan 18 & 120 & clear & 0.6 & $0.8 \times 15$ & prism & 75 & 1.34 & HD 32090 \\
\hline HIP 32728 B & 2013 Apr 3 & 360 & clear & 0.7 & $0.8 \times 15$ & prism & 75 & 1.05 & HD 56386 \\
\hline HIP 37283 B & 2012 Nov 16 & 240 & thin clouds & 0.4 & $0.8 \times 15$ & SXD & 750 & 1.18 & HD 63586 \\
\hline HIP 46984 B & 2013 Apr 5 & 480 & clouds & 0.5 & $0.8 \times 15$ & prism & 75 & 1.21 & HD 101369 \\
\hline HIP 49046 B & 2012 Nov 16 & 360 & thin clouds & 0.6 & $0.8 \times 15$ & SXD & 750 & 1.04 & HD 89239 \\
\hline HIP 51877 B & 2014 Jan 17 & 180 & clear & 0.5 & $0.8 \times 15$ & prism & 75 & 1.10 & HD 71906 \\
\hline HIP 52463 B & 2013 Apr 3 & 360 & clear & 0.7 & $0.8 \times 15$ & prism & 75 & 1.28 & HD 90606 \\
\hline HIP 55666 B & 2011 Apr 20 & 80 & clear & 0.5 & $0.5 \times 15$ & prism & 120 & 1.509 & HD 97585 \\
\hline HIP 58918 B & 2013 Apr 16 & 360 & clouds & 0.5 & $0.8 \times 15$ & prism & 75 & 1.14 & HD 105388 \\
\hline HIP 59310 B & 2013 Apr 16 & 360 & clouds & 0.5 & $0.8 \times 15$ & prism & 75 & 1.04 & HD 105388 \\
\hline HIP 59933 B & 2011 Mar 31 & 480 & clear & 0.3 & $0.5 \times 15$ & prism & 120 & 1.08 & HD 10538 \\
\hline HIP $60501 \mathrm{~B}$ & 2011 Apr 20 & 80 & clear & 0.5 & $0.5 \times 15$ & prism & 120 & 1.208 & HD 109309 \\
\hline HIP 60987 B & 2013 Apr 16 & 360 & cloudy & 0.4 & $0.8 \times 15$ & prism & 75 & 1.14 & HD 101060 \\
\hline HIP 60987 C & 2013 Apr 16 & 360 & cloudy & 0.4 & $0.8 \times 15$ & prism & 75 & 1.16 & HD 101060 \\
\hline HIP $63506 \mathrm{C}$ & 2013 Jan 26 & 360 & clear & 0.7 & $0.8 \times 15$ & prism & 75 & 1.083 & HD 109615 \\
\hline HIP 65706 B & 2012 Apr 30 & 180 & fog & 1.5 & $0.8 \times 15$ & prism & 75 & 1.4 & HD 116960 \\
\hline HIP $65780 \mathrm{C}$ & 2013 Jul 14 & 180 & clear & 0.9 & $0.8 \times 15$ & prism & 75 & 1.73 & HD 116960 \\
\hline HIP $70623 \mathrm{C}$ & 2012 Jul 7 & 720 & clear & 0.5 & $0.5 \times 15$ & SXD & 1200 & 1.11 & HD 132072 \\
\hline HIP 75310 B & 2013 Apr 17 & 720 & clear & 0.7 & $0.8 \times 15$ & SXD & 750 & 1.30 & HD 144425 \\
\hline HIP 76456 B & 2013 Apr 5 & 120 & cloudy & 0.8 & $0.8 \times 15$ & prism & 75 & 1.26 & HD 40739 \\
\hline HIP 76641 B & 2013 Apr 3 & 90 & clear & 0.9 & $0.8 \times 15$ & prism & 75 & 1.3 & HD 136831 \\
\hline HIP 78184 B & 2011 Mar 31 & 160 & clear & 0.3 & $0.5 \times 15$ & prism & 120 & 1.37 & HD 145127 \\
\hline HIP 78859 B & 2012 Aug 10 & 180 & clear & 0.5 & $0.5 \times 15$ & SXD & 1200 & 1.40 & q Her \\
\hline HIP 78916 B & 2013 Jul 13 & 180 & clear & 0.5 & $0.8 \times 15$ & prism & 75 & 1.07 & HD 165029 \\
\hline HIP 78923 B & 2013 Jul 13 & 180 & clear & 0.5 & $0.8 \times 15$ & prism & 75 & 1.14 & HD 145647 \\
\hline HIP $79180 \mathrm{~B}$ & 2013 Apr 3 & 360 & clear & 0.9 & $0.8 \times 15$ & prism & 75 & 1.44 & HD 172728 \\
\hline HIP 80258 B & 2012 Aug 10 & 50 & thin clouds & 0.4 & $0.8 \times 15$ & prism & 75 & 1.2 & $26 \mathrm{Ser}$ \\
\hline HIP 81910 B & 2011 Apr 20 & 80 & clear & 0.5 & $0.5 \times 15$ & prism & 120 & 1.485 & HD 145127 \\
\hline HIP 82233 B & 2011 Apr 20 & 80 & clear & 0.5 & $0.5 \times 15$ & prism & 120 & 1.276 & HD 148968 \\
\hline HIP 83651 B & 2012 Jul 11 & 100 & thin clouds & 0.9 & $0.8 \times 15$ & prism & 75 & 1.1 & HD 159008 \\
\hline HIP 85365 B & 2012 Jul 7 & 600 & clear & 0.5 & $0.8 \times 15$ & SXD & 750 & 1.25 & HD 159415 \\
\hline HIP 86722 B & 2011 Apr 20 & 80 & clear & 0.5 & $0.5 \times 15$ & prism & 120 & 1.006 & HD 165029 \\
\hline HIP 88728 B & 2012 Jul 6 & 20 & clear & 1.3 & $0.3 \times 15$ & prism & 200 & 1.23 & HD 165029 \\
\hline HIP 90273 B & 2013 Nov 8 & 200 & cloudy & 1.3 & $0.3 \times 15$ & prism & 200 & 1.17 & HD 174567 \\
\hline HIP 90869 B & 2012 Aug 10 & 80 & clear & 0.3 & $0.5 \times 15$ & SXD & 1200 & 1.51 & HD 184533 \\
\hline HIP 93967 B & 2011 Oct 14 & 300 & cloudy & 1.0 & $0.8 \times 15$ & prism & 75 & 1.28 & HD 182678 \\
\hline HIP 97168 B & 2012 Jul 6 & 40 & clear & 1.3 & $0.3 \times 15$ & prism & 200 & 1.15 & HD 199217 \\
\hline HIP 98535 C & 2012 Jul 6 & 120 & clear & 1.3 & $0.3 \times 15$ & prism & 200 & 1.14 & HD 199217 \\
\hline HIP 102582 B & 2013 Jul 13 & 60 & clear & 0.5 & $0.8 \times 15$ & prism & 75 & 1.08 & HD 192538 \\
\hline HIP 103199 B & 2012 Jul 6 & 120 & clear & 1.3 & $0.3 \times 15$ & prism & 200 & 1.046 & HD 210501 \\
\hline HIP 105202 B & 2012 Jul 6 & 150 & clear & 1.3 & $0.3 \times 15$ & prism & 200 & 1.043 & HD 210501 \\
\hline HIP $106551 \mathrm{~B}$ & 2012 Aug 11 & 240 & clear & 0.7 & $0.5 \times 15$ & prism & 120 & 1.245 & HD199312 \\
\hline HIP 108822 B & 2012 Aug 11 & 80 & clear & 0.7 & $0.5 \times 15$ & prism & 120 & 1.247 & HD 210501 \\
\hline HIP 109454 B & 2011 Oct 15 & 1440 & clear & 0.6 & $0.8 \times 15$ & prism & 75 & 1.04 & HD 210501 \\
\hline HIP 111657 B & 2012 Oct 2 & 240 & cloudy & 0.8 & $0.8 \times 15$ & SXD & 750 & 1.19 & HD 210501 \\
\hline HIP 112422 B & 2012 Oct 7 & 540 & thin cloud & 0.9 & $0.8 \times 15$ & prism & 75 & 1.03 & HD 210501 \\
\hline HIP 114424 B & 2012 Jul 7 & 140 & clear & 0.5 & $0.5 \times 15$ & prism & 120 & 1.163 & HD 218639 \\
\hline HIP 114456 B & 2012 Oct 2 & 90 & cloudy & 0.7 & $0.8 \times 15$ & SXD & 750 & 1.24 & HD 219290 \\
\hline HIP $115819 \mathrm{~B}$ & 2012 Jul 6 & 240 & clear & 1.3 & $0.8 \times 15$ & prism & 75 & 1.198 & HD 203769 \\
\hline HIP 116052 B & 2012 Oct 25 & 420 & cloudy & 0.6 & $0.8 \times 15$ & SXD & 750 & 1.01 & HD 210501 \\
\hline \multicolumn{10}{|c|}{ Non-Hipparcos Companions } \\
\hline NLTT 1011 B & 2013 Sep 23 & 720 & clear & 0.6 & $0.8 \times 15$ & prism & 75 & 1.04 & HD 210501 \\
\hline GD $280 \mathrm{~B}$ & 2013 Dec 11 & 720 & cloudy & 1.2 & $0.8 \times 15$ & prism & 75 & 1.30 & HD 15090 \\
\hline NLTT 8245 B & 2014 Jan 19 & 180 & clear & 0.7 & $0.8 \times 15$ & prism & 75 & 1.51 & HD 10897 \\
\hline LSPM J0241+2553 B & 2014 Jan 18 & 960 & clear & 0.6 & $0.8 \times 15$ & prism & 75 & 1.22 & HD 22401 \\
\hline HD 253662 B & 2014 Jan 18 & 360 & clear & 0.6 & $0.8 \times 15$ & prism & 75 & 1.13 & HD 43583 \\
\hline
\end{tabular}


Table 8

(Continued)

\begin{tabular}{|c|c|c|c|c|c|c|c|c|c|}
\hline Object & $\begin{array}{l}\text { Date } \\
\text { (UT) }\end{array}$ & $\begin{array}{l}t_{\text {int }} \\
(\mathrm{s})\end{array}$ & Conditions & $\begin{array}{c}\text { Seeing } \\
\left({ }^{\prime \prime}\right)\end{array}$ & $\begin{array}{l}\text { Slit } \\
\left({ }^{\prime \prime}\right)\end{array}$ & Mode & $\begin{array}{c}\text { Resolution } \\
\lambda / \Delta \lambda\end{array}$ & Airmass & Standard \\
\hline LSPM J0632+5053 B & 2013 Apr 4 & 480 & clear & 0.7 & $0.8 \times 15$ & prism & 75 & 1.30 & HD 248790 \\
\hline NLTT 18587 B & 2014 Jan 19 & 960 & clear & 0.7 & $0.8 \times 15$ & prism & 75 & 1.07 & HD 58296 \\
\hline NLTT 19109 B & 2014 Jan 18 & 240 & clear & 0.6 & $0.8 \times 15$ & prism & 75 & 1.35 & HD 73687 \\
\hline NLTT 22073 B & 2013 Nov 23 & 160 & clear & 1.1 & $0.8 \times 15$ & prism & 75 & 1.49 & HD 87727 \\
\hline NLTT 23716 B & 2014 Jan 19 & 80 & clear & 0.7 & $0.8 \times 15$ & prism & 75 & 1.12 & HD 79108 \\
\hline NLTT 26746 B & 2011 Mar 31 & 480 & clear & 0.3 & $0.5 \times 15$ & prism & 120 & 1.05 & HD 105388 \\
\hline NLTT 29395 B & 2014 Jan 19 & 360 & clear & 0.7 & $0.8 \times 15$ & prism & 75 & 1.28 & HD 128039 \\
\hline NLTT 30510 B & 2014 Jan 19 & 240 & clear & 0.7 & $0.8 \times 15$ & prism & 75 & 1.05 & HD 109615 \\
\hline NLTT 31450 B & 2011 Mar 31 & 480 & clear & 0.3 & $0.5 \times 15$ & prism & 120 & 1.03 & HD 105388 \\
\hline PMI 13410+0542 B & 2013 Jul 13 & 360 & clear & 0.9 & $0.8 \times 15$ & prism & 75 & 1.12 & HD 116960 \\
\hline PMI 13518+4157 B & 2013 Jul 13 & 360 & clear & 0.9 & $0.8 \times 15$ & prism & 75 & 1.14 & HD 116960 \\
\hline NLTT 38489 B & 2013 Apr 18 & 720 & clear & 0.5 & $0.8 \times 15$ & prism & 75 & 1.45 & HD 143187 \\
\hline NLTT 39312 B & 2013 Jul 13 & 360 & clear & 0.9 & $0.8 \times 15$ & prism & 75 & 1.18 & HD 136831 \\
\hline LSPM J1627+3328 B & 2013 Apr 17 & 360 & cloudy & 0.7 & $0.8 \times 15$ & prism & 75 & 1.12 & HD 145647 \\
\hline NLTT 44368 B & 2013 Apr 16 & 360 & thin clouds & 0.7 & $0.8 \times 15$ & prism & 75 & 1.27 & HD 199217 \\
\hline LSPM J1717+5925 B & 2013 Jul 12 & 1200 & clear & 1.0 & $0.8 \times 15$ & prism & 75 & 1.45 & HD 179933 \\
\hline NLTT 52268 B & 2013 Jul 12 & 120 & clear & 0.8 & $0.8 \times 15$ & prism & 75 & 1.08 & HD 210501 \\
\hline LSPM J2153+1157 B & 2013 Jul 12 & 180 & clear & 0.8 & $0.8 \times 15$ & prism & 75 & 1.10 & HD 210501 \\
\hline PM I22118-1005 B & 2013 Jul 12 & 720 & clear & 0.8 & $0.8 \times 15$ & prism & 75 & 1.18 & HD 203769 \\
\hline NLTT $55219 \mathrm{~B}$ & 2013 Jul 12 & 720 & clear & 0.8 & $0.8 \times 15$ & prism & 75 & 1.04 & HD 210501 \\
\hline \multicolumn{10}{|c|}{ Serendipitous Companion Discoveries } \\
\hline NLTT 27966 B & 2012 Jun 7 & 360 & clear & 1.0 & $0.8 \times 15$ & prism & 75 & 1.16 & HD 99966 \\
\hline LSPM J1336+2541 B & 2012 Jul 8 & 960 & clear & 0.9 & $0.8 \times 15$ & prism & 75 & 1.06 & HD 116960 \\
\hline HIP 73169 B & $2012 \mathrm{Jul} 6$ & 960 & clear & 1.0 & $0.8 \times 15$ & prism & 75 & 1.30 & HD 116960 \\
\hline PM 23492+3458 B & $2012 \mathrm{Jul} 7$ & 720 & clear & 0.6 & $0.8 \times 15$ & prism & 75 & 1.31 & HD 203030 \\
\hline \multicolumn{10}{|c|}{ Unlikely companions } \\
\hline NLTT 35593 B & 2013 Apr 18 & 360 & clear & 0.5 & $0.8 \times 15$ & prism & 75 & 1.40 & HD 116960 \\
\hline \multicolumn{10}{|c|}{ Primary Stars } \\
\hline NLTT 730 & 2013 Sep 23 & 180 & clear & 0.7 & $0.8 \times 15$ & SXD & 750 & 1.03 & HD 210501 \\
\hline LSPMJ0632+5053 & 2013 Apr 17 & 90 & patchy cloud & 0.7 & $0.3 \times 15$ & SXD & 2000 & 1.24 & HD 39250 \\
\hline NLTT 26746 & 2012 Jul 5 & 280 & clear & 1.2 & $0.3 \times 15$ & SXD & 2000 & 1.30 & HD 99966 \\
\hline NLTT 27966 & $2012 \mathrm{Jul} 5$ & 720 & clear & 1.2 & $0.3 \times 15$ & SXD & 2000 & 1.56 & HD 101060 \\
\hline NLTT 31450 & 2012 Jul 5 & 240 & clear & 0.8 & $0.3 \times 15$ & SXD & 2000 & 1.30 & HD 118214 \\
\hline LSPMJ1336+2541 & 2013 Jul 12 & 90 & clear & 1.0 & $0.3 \times 15$ & SXD & 2000 & 1.10 & HD 109691 \\
\hline PM I13518+4157 & 2013 Jul 12 & 90 & clear & 1.0 & $0.3 \times 15$ & SXD & 2000 & 1.06 & HD 123233 \\
\hline HIP 83651 & 2013 Apr 17 & 90 & cloudy & 0.7 & $0.8 \times 15$ & SXD & 750 & 1.11 & HD 167163 \\
\hline HIP 84840 & 2013 Apr 16 & 180 & cloudy & 0.8 & $0.3 \times 15$ & SXD & 2000 & 1.07 & HD 165029 \\
\hline NLTT 44368 & $2013 \mathrm{Jul} 12$ & 90 & clear & 0.8 & $0.3 \times 15$ & SXD & 2000 & 1.32 & HD 199217 \\
\hline HIP 97168 & 2013 Apr 17 & 90 & cloudy & 0.7 & $0.8 \times 15$ & SXD & 750 & 1.20 & HD 197291 \\
\hline HIP 108822 & $2013 \mathrm{Jul} 12$ & 90 & clear & 1.0 & $0.3 \times 15$ & SXD & 2000 & 1.08 & HD 210501 \\
\hline PM I22118-1005 & 2013 Jul 12 & 90 & clear & 1.0 & $0.3 \times 15$ & SXD & 2000 & 1.25 & HD 203893 \\
\hline PM I23492+3458 & 2013 Jul 12 & 90 & clear & 1.0 & $0.3 \times 15$ & SXD & 2000 & 1.31 & HD 219290 \\
\hline
\end{tabular}

(2007) SEDs. We calculated the errors in distance caused by an uncertainty in spectral type, by the rms of the fits, and by the error on the 2MASS photometry. We then calculated the weighted mean of these distance estimates, weighting only by our measurement errors, i.e., the quadrature sum of the error in the photometric measurements and the propagated error in spectral typing. We then calculated a final error on this distance based on the quadrature sum of the photometric error, intrinsic scatter, and propagated error in spectral type for each band. Hence the final quoted error includes both the effects of measurement errors and the rms intrinsic scatter about the photometric relations. The calculated photometric distances are shown in Tables 4 and 5. Note that our white dwarf companion (HIP 88728 B), which was saturated in Pan-STARRS 1, is not resolved in plate images.
We also calculated photometric distances for our primaries that lacked trigonometric parallaxes. For primaries with measured spectral types, we applied a similar process as for our secondaries. Where there were no literature spectral distances we used relations from Lépine (2005) and the errors in those distances were calculated in the same way as for the secondaries. For our two white dwarf primaries we used the photometric distance relations of Limoges et al. (2013). For this we assumed a DA spectral type and a mass of $0.6 M_{\odot}$. Limoges et al. (2013) quote a photometric distance error of $15 \mathrm{pc}$ for their sample with approximate distances of $30 \mathrm{pc}$. Hence we assume errors of $50 \%$ for our white dwarf photometric distances. It appears that most of our companions have photometric distances that are in good agreement with the distances to their primaries. This is shown in Figure 10. 
Table 9

Spectroscopic Classification of Our L Dwarf Companions

\begin{tabular}{|c|c|c|c|c|c|c|c|c|}
\hline Object & SpT(Visual) & $\mathrm{H}_{2} O \_\mathrm{J}$ & $\mathrm{SpT}\left(\mathrm{H}_{2} O_{-} \mathrm{J}\right)$ & $\mathrm{H}_{2} O \_\mathrm{H}$ & $\mathrm{SpT}\left(\mathrm{H}_{2} O_{-} \mathrm{H}\right)$ & $\mathrm{CH}_{4 \_} \mathrm{K}$ & $\mathrm{SpT}\left(\mathrm{CH}_{4} \_\mathrm{K}\right)$ & $\begin{array}{c}\text { SpT(Final) } \\
\text { Type }\end{array}$ \\
\hline \multicolumn{9}{|c|}{ HIP search companions } \\
\hline HIP 6407B & L0 & 0.831 & L3.3 & 0.776 & L3.6 & 1.002 & L4.3 & $\mathrm{L} 1+\mathrm{T} 5^{\mathrm{c}}$ \\
\hline HIP 2397B & L0 & 0.935 & L0.6 & 0.846 & L0.8 & 1.053 & $\mathrm{~L} 2.3$ & L0.5 \\
\hline HIP 59933B & L1.5 & 0.922 & L0.9 & 0.838 & L1.1 & 1.042 & $\mathrm{~L} 2.8$ & L1 \\
\hline HIP $63506 \mathrm{C}$ & $\mathrm{L}^{\mathrm{a}}$ & 0.909 & $\mathrm{~L} 1.2$ & 0.82 & L1.8 & 1.025 & L3.5 & L1 \\
\hline HIP 85365B & L5 & 0.752 & L5.7 & 0.701 & L6.5 & 0.965 & L5.6 & L5.5 \\
\hline HIP 9269B & L6 & 0.752 & L5.7 & 0.781 & L3.3 & 0.978 & L5.2 & L6 \\
\hline HIP 11161B & L1.5 & 0.900 & L1.4 & 0.834 & $\mathrm{~L} 1.2$ & 1.01 & L4.0 & L1.5 \\
\hline HIP 112422B & L2 & 0.809 & L4.0 & 0.837 & L1.1 & 1.028 & L3.3 & L1.5 \\
\hline \multicolumn{9}{|c|}{ Companions to faint non-HIP primaries } \\
\hline NLTT 1011B & L1.5 & 0.843 & L2.9 & 0.763 & L4.0 & 1.01 & L 4.2 & L2 \\
\hline NLTT 55219B ${ }^{\mathrm{b}}$ & L2 & 0.793 & L4.5 & 0.707 & L6.3 & 0.943 & L6.3 & L5.5 \\
\hline PM I22118-1005 B & $\mathrm{L} 1.5$ & 0.906 & $\mathrm{~L} 1.3$ & 0.806 & $\mathrm{~L} 2.3$ & 1.03 & L3.1 & $\mathrm{L} 1.5$ \\
\hline PM I13410+0542B & L4 & 0.852 & $\mathrm{~L} 2.7$ & 0.734 & L5 5 & 1.115 & M9.3 & $\mathrm{L} 4$ \\
\hline PM I13518+4157B & L1.5 & 0.882 & L1.8 & 0.829 & L1.4 & 0.985 & L4.9 & L1.5 \\
\hline LSPM J0241+2553 B & $\mathrm{L} 1.5$ & 0.922 & L0.9 & 0.841 & L0.9 & 1.064 & $\mathrm{~L} 1.8$ & $\mathrm{~L} 1$ \\
\hline HD 253662 B & L1.5 & 0.942 & L0.5 & 0.0866 & L0.0 & 1.060 & $\mathrm{~L} 2.0$ & L0.5 \\
\hline LSPM J0632+5053B & L2 & 0.919 & L1.0 & 0.846 & L0.7 & 0.999 & L4.4 & $\mathrm{L} 1.5$ \\
\hline NLTT 26746B & L4 & 0.786 & L4.7 & 0.721 & L5.7 & 1.031 & $\mathrm{~L} 3.2$ & L4 \\
\hline NLTT 31450B & L6 & 0.829 & L3.4 & 0.784 & $\mathrm{~L} 3.2$ & 1.057 & $\mathrm{~L} 2.1$ & L6 \\
\hline NLTT 44368B & L1.5 & 0.899 & L1.4 & 0.834 & $\mathrm{~L} 1.2$ & 1.038 & L2.9 & L1.5 \\
\hline LSPM J1717+5925 B & $\mathrm{L} 1.5$ & 0.953 & L0.3 & 0.850 & L0.6 & 1.042 & $\mathrm{~L} 2.8$ & L1 \\
\hline \multicolumn{9}{|c|}{ Serendipitous companion discoveries } \\
\hline NLTT 27966B & L4 & 0.771 & L5.1 & 0.718 & L5.9 & 1.011 & $\mathrm{~L} 4.0$ & L4 \\
\hline LSPM J1336+2541B & L4 & 0.848 & $\mathrm{~L} 2.8$ & 0.718 & L5.9 & 0.964 & L5.6 & $\mathrm{L} 4$ \\
\hline HIP 73169B & $\mathrm{L} 2.5$ & 0.87 & $\mathrm{~L} 2.2$ & 0.787 & L3.1 & 1.059 & $\mathrm{~L} 2.0$ & $\mathrm{~L} 2.5$ \\
\hline PM 23492+3458B & L9 & 0.615 & L9.7 & 0.63 & L9.0 & 0.79 & L9.7 & L9 \\
\hline \multicolumn{9}{|c|}{ Unlikely discoveries } \\
\hline NLTT 35593B & L2 & 0.878 & $\mathrm{~L} 2.0$ & 0.796 & $\mathrm{~L} 2.8$ & 1.049 & $\mathrm{~L} 2.5$ & $\mathrm{~L} 2$ \\
\hline
\end{tabular}

Notes.

a No good standard comparison was found; the best match in the SpeX prism library was the L1 2MASSWJ143928.4+192915 (Burgasser et al. 2004).

b No good standard comparison was found; the best match in the SpeX prism library was the L5.5 2MASS J17502484-0016151 (Kendall et al. 2007).

${ }^{\mathrm{c}}$ Using spectral decomposition we determined that HIP 6407B is likely itself a double consisting of an L1 and a T5. We later resolved this companion as a tight binary itself. See Section 5.2.1 for more details.

\subsection{Spectral Types and Ages of the Primaries}

\subsubsection{Spectral Types}

We searched the literature for information on our primary stars. While many had spectral types either from previous measurements, 32 were unclassified. For primaries with no published spectral type we have obtained where possible nearinfrared or optical spectroscopic observations (see Sections 3.2 and 3.3). These objects are shown in Figures 5 and 11. For objects with no spectral type from the literature or that were not observed as part of our follow-up program, we used the $V-J$ to spectral type relation of Lépine \& Gaidos (2011). For our non-Hipparcos primaries, we use the $M_{V}$ magnitudes listed in Lépine \& Shara (2005) and Salim \& Gould (2003) along with $J$ magnitudes from 2MASS. For the single object that was too blue for this relation to be valid (HIP 111657), we used the primary's 2MASS photometry and the SED of Kraus \& Hillenbrand (2007) to estimate spectral type. Tables 10 and 11 show details of our primary stars.
For objects observed with SNIFS, spectral types for NLTT 730, LSPM J2153+1157, and NLTT 22073 were determined following the methods outlined in Lépine et al. (2013). Specifically, we measured the strengths of the TiO and $\mathrm{CaH}$ features and then compared to stars from Reid et al. (1995). We also matched each spectrum by eye to templates from Bochanski et al. (2007) using the IDL spectral typing suite of Covey et al. (2007). Metallicities were determined following the methods of Mann et al. (2013a), which provide empirical relations between visible-wavelength features and metallicities for late- $\mathrm{K}$ to mid-M dwarfs. Errors in metallicities were calculated considering both errors in the Mann et al. (2013a) calibration and measurement errors. We classified NLTT 1011 using our Mayall Telescope data and the Covey et al. (2007) HAMMER indices, resulting in a type of K5. Our other Kitt Peak targets, NLTT 38489 and HIP 84840 B, were visually compared to the spectral templates of Bochanski et al. (2007) with best matches of M3 and M4, respectively; these subtypes were confirmed by index measurements using the method of Lépine et al. (2013) for NLTT 38489, while HIP 

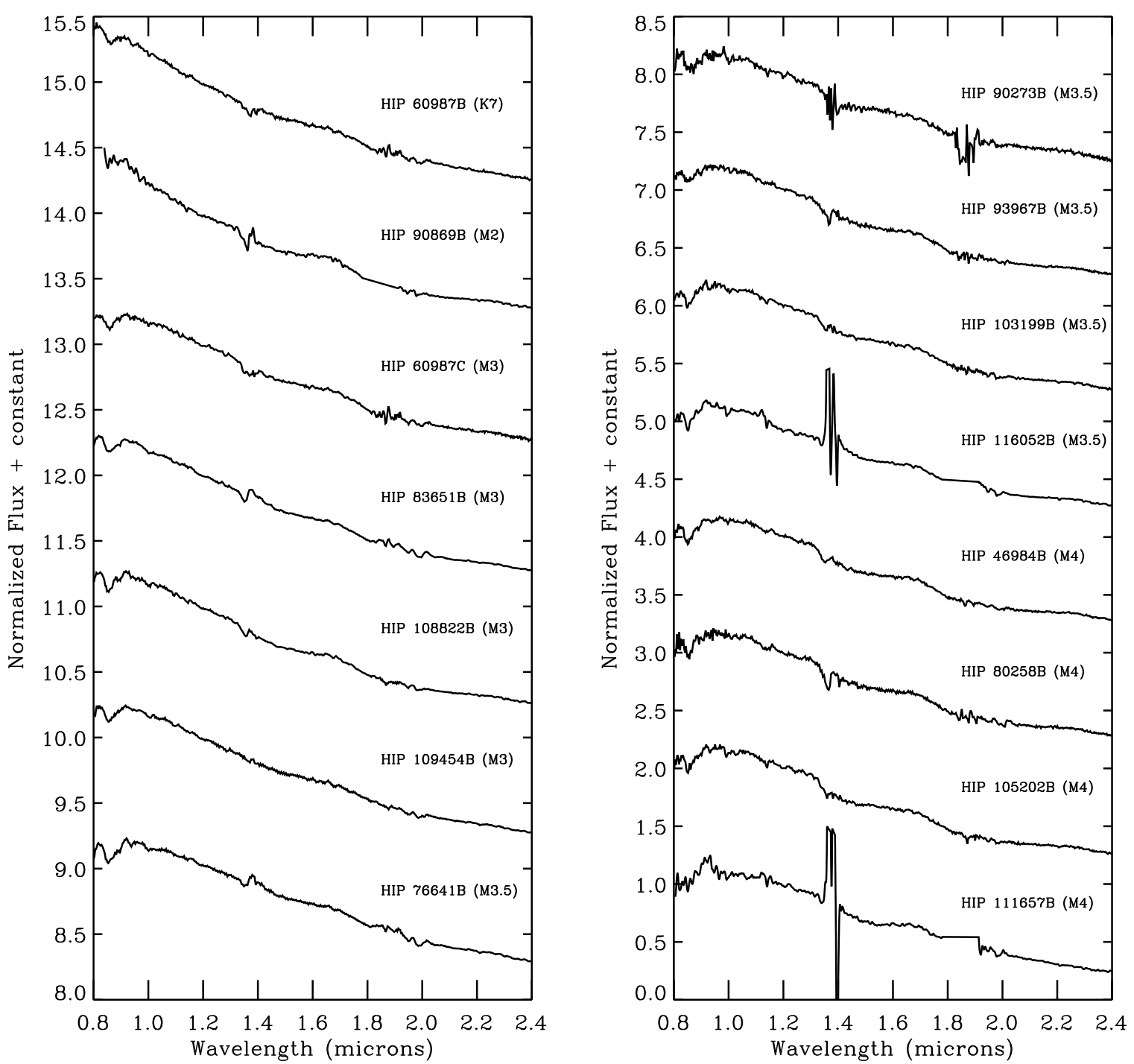

Figure 1. IRTF/SpeX spectra for our Hipparcos companions with spectral types from M0.5 to M4. Spectra taken with SpeX SXD mode have been Gaussian smoothed to $R=200$. For the SXD spectra note that the noisy gaps at $1.4 \mu \mathrm{m}$ and $1.8 \mu \mathrm{m}$ are caused by the order boundaries.

84840 B was classified as an M3.5. We adopt our visual comparisons along with a spectral typing error of half a subclass for these two objects. None of our observed objects showed emission in $\mathrm{H} \alpha$. One of our SNIFS targets, GD 280, was listed as a candidate white dwarf by Giclas et al. (1967). Our spectrum shows clear Balmer line absorption; hence we classify it as a DA white dwarf. We do not have a spectrum for LSPM J0241+2553. The resulting spectral types and metallicities for the objects observed with SNIFS are listed in Table 11 and the spectra are shown in Figure 11.

\subsubsection{Age Estimates}

Twenty-three of our primary stars have age estimates listed in the literature. For remaining objects with no published ages, we used archival $\mathrm{Ca} \mathrm{H}$ and $\mathrm{K}$ emission and where this was found, applied the age-activity relation of Mamajek \& Hillenbrand (2008). The majority of our primaries had no such data, and the only object that did had no age derived from such emission in the literature. For the remaining 54 objects with no $\mathrm{Ca} \mathrm{H}$ and $\mathrm{K}$ measurements, we set an approximate upper age limit of $\sim 10 \mathrm{Gyr}$ based on their disk-like kinematics.
To set a lower limit for the handful of M-dwarf primaries where we had an optical spectrum, we used the object's lack of $\mathrm{H} \alpha$ and the activity lifetime of West et al. (2008). Here we used the $1 \sigma$ lower limit for the activity age, taking into account that our spectral types have an uncertainty of 0.5 spectral types. For all our objects without $\mathrm{H} \alpha$ or $\mathrm{Ca} \mathrm{H}$ and $\mathrm{K}$ data, we searched the ROSAT Faint Source catalog (Voges et al. 2000) with a matching radius of 30". We identified four objects as having weak X-ray emission; the rest we assumed that the flux was below the limiting flux quoted by Schmitt et al. (1995). We then used the distances to these objects along with the counts to flux conversion of Schmitt et al. (1995) to estimate the X-ray luminosity. We converted this to the X-ray to bolometric luminosity ratio $\left(R_{x}\right)$ using an $L_{\text {bol }}$ calculated from Tycho photometry (Hog et al. 2000), the color relations of Mamajek et al. (2002), and the bolometric corrections of Pecaut \& Mamajek (2013). This was then applied to the ageto-X-ray activity relation of Mamajek \& Hillenbrand (2008) to obtain lower age limits. Note that this X-ray relation has not been calibrated beyond mid-K spectral types, hence we used the objects' lack of X-ray emission to set a lower age limit of $300 \mathrm{Myr}$ for objects of this spectral type. This is the age below 

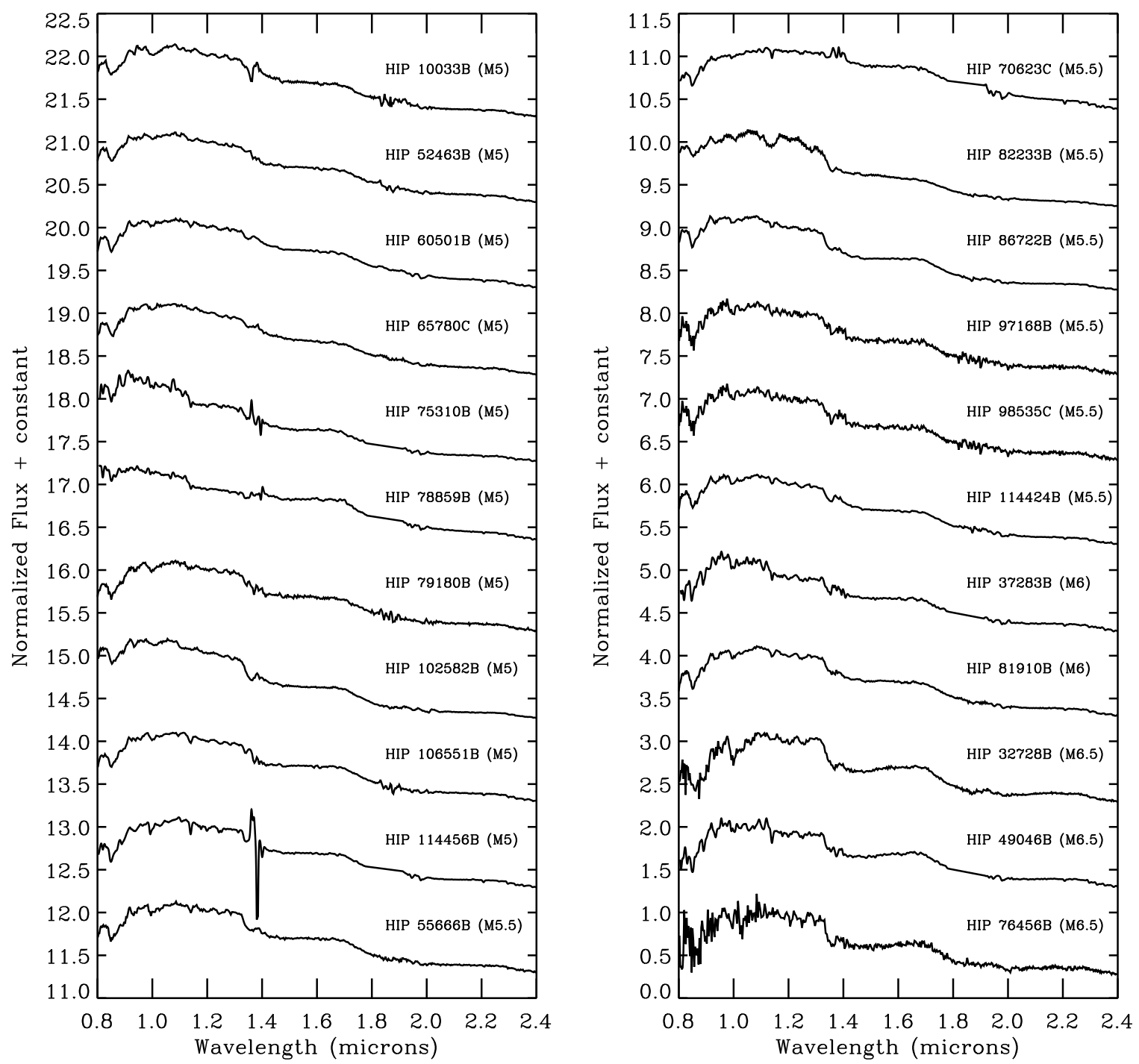

Figure 2. IRTF/SpeX spectra for our Hipparcos companions with spectral types from M5 to M6.5. Spectra taken with SpeX SXD mode have been Gaussian smoothed to $R=200$.

which low mass stars show significant X-ray emission (Shkolnik et al. 2009).

\section{DISCUSSION}

\subsection{Comparison with the Field Ultracool Wide Binary Population}

Our sample contains 24 new L-dwarf companions to main sequence stars (including the previously typed but unrecognized L7 companion to NLTT 730 but not the unlikely L2 companion to NLTT 35593). Additionally we identified 21 new wide M-dwarf companions with spectral types of M7 or later and typed two previously proposed companions.

In order to study the wide ultracool (spectral type $\geqslant M 7$ ) binary population, we compiled a list of wide ultracool companions to stars (see Table 12). We began with the compilation of Faherty et al. (2010; excluding the companion to NLTT 20346 that Dupuy \& Liu 2012 concluded was not physically related due to its high proper motion difference from its primary) and added spectroscopically confirmed objects from the literature discovered since then. We do not include objects that have been identified as candidate binaries but that lack spectral types such as from the studies of Deacon et al. (2009), Dhital et al. (2010), and Smith et al. (2014). Figure 12 shows the spectral type of the secondary plotted against the projected separation $\left(r_{\mathrm{AU}}\right)$. At first glance, there is an apparent scarcity of $\mathrm{T}$ dwarf companions wider than $3000 \mathrm{AU}$. In fact, this is due to previous efforts focusing on identifying close companions and from the known population being drawn from a series of heterogeneous surveys. Figure 13 shows a histogram comparing the combined contribution of this work, Deacon et al. (2012b) and Deacon et al. (2012a) to the total number of companions. This paper's contribution is most significant beyond $\log _{10} r_{\mathrm{AU}}=3.5$ where we have doubled the population of L dwarf companions. In total we have increased the wide ( $>300 \mathrm{AU}$ ) L dwarf companion population by $82 \%$ and doubled the number of ultracool $\mathrm{M}$ dwarf companions in the same range. While the $\mathrm{L}$ dwarf population exhibits an approximately flat distribution in $\log _{10} r_{\mathrm{AU}}$, the $\mathrm{T}$ dwarf companion population peaks between $\log _{10} r_{\mathrm{AU}}=3.0$ and 3.5. However, any claim of a preferred separation for $\mathrm{T}$ dwarf companions (or of a log-flat distribution for $\mathrm{L}$ dwarfs) should be treated with caution as the sample is drawn from a disparate set of surveys. Similarly the apparent cut-off above $10,000 \mathrm{AU}$ is likely due to incompleteness in surveys (including our own) and the difficulty of disentangling the widest binaries from coincident alignments from field stars. In a future paper 

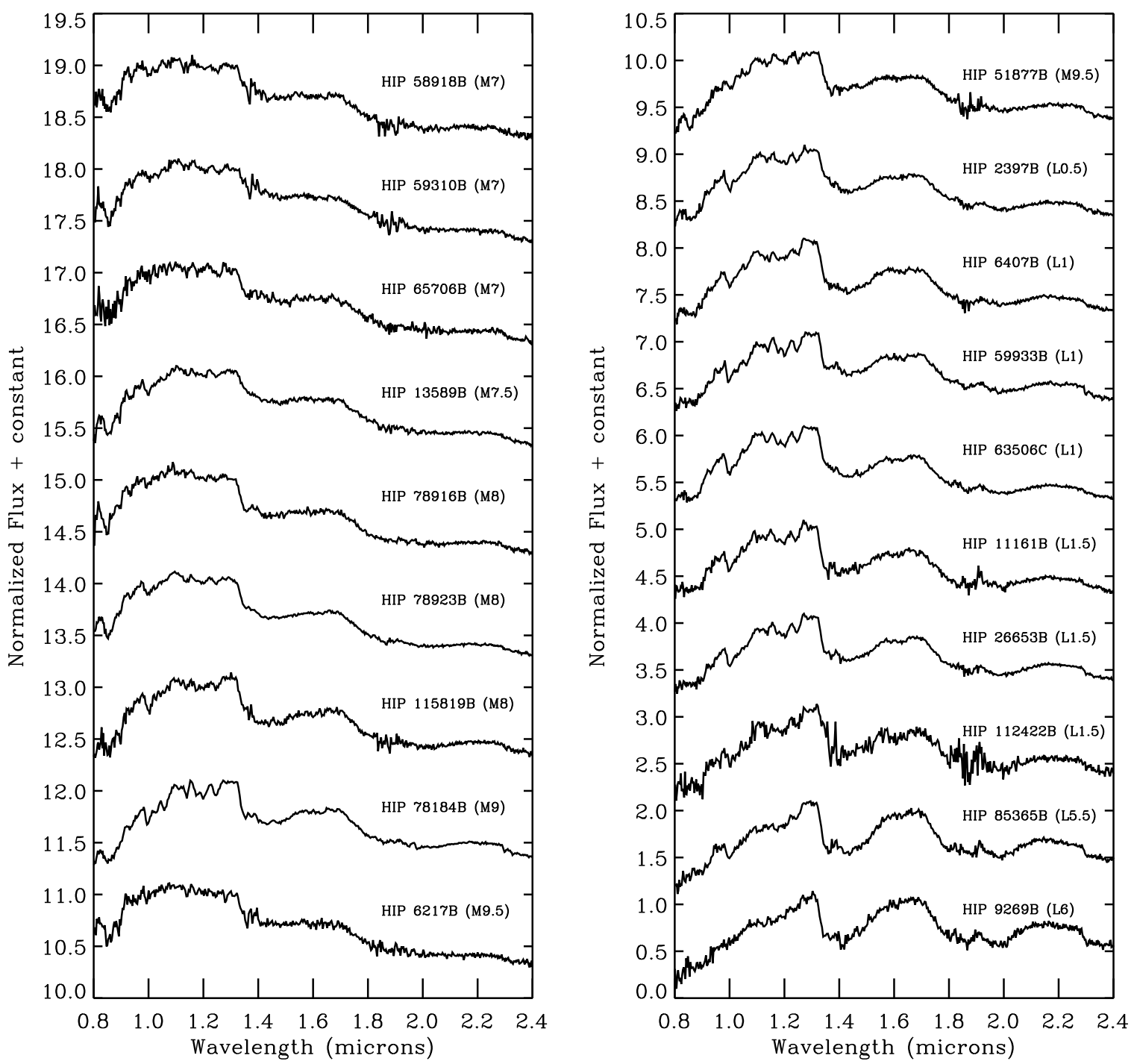

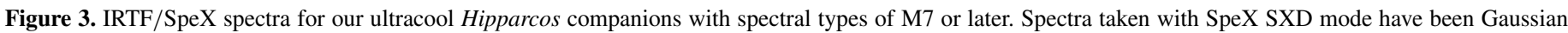
smoothed to $R=200$. Note that HIP 6407 B was resolved as being an L1+T3 binary itself. See Section 5.2.1 for details.

we aim to take the final results of our survey, model our selection biases, and determine the true separation distribution of the ultracool companion population.

In order to determine the stability of the population of systems containing a wide, ultracool companion, we estimated the total mass of each system. Where an object had a mass quoted in the literature, we used that value. For known $\mathrm{L}$ and $\mathrm{T}$ dwarfs with no quoted mass estimates and our new $\mathrm{L}$ and $\mathrm{T}$ companions, we used a value of $0.075 M_{\odot}$, in essence making our total mass estimates for these systems upper limits. For A-M stars with no mass in the literature, we converted their spectral type to mass using the relations of Kraus \& Hillenbrand (2007). ${ }^{15}$ The results are plotted in Figure 14 along with the maximum separation versus total mass relations suggested by Close et al. (2003) and Reid et al. (2001). It is clear that a substantial number of latetype companions lie outside both of these suggested maximum boundaries and are hence loosely bound. Dhital et al. (2010) used a simple model of interactions in the Galactic disk (based

15 For the LSPM J1202+0742 ABC system (Smith et al. 2014), we estimated the spectral type of the brighter component based on their $V-J$ color (M0, LSPM J1202+0742N) or 2MASS photometry (M1, LSPM J1202+0742S). We then used these to determine the total mass of the system. on Weinberg et al. 1987) to calculate the typical maximum separation for a given system age and total mass. This is also plotted in Figure 14 for a number of different ages. It is clear that, though loosely bound, very few of the widest systems would be disrupted over the lifetime of the Galactic disk.

\subsection{Interesting Individual Systems \\ 5.2.1. HIP 6407Bab}

We initially classified HIP 6407B as an L0 based on visual comparison to standards. However, the object has significantly stronger water absorption features suggestive of an object two or three subtypes later. As this may result from the contribution of an unresolved additional component, we observed this object on 2013 October 14 UT at the Keck II telescope using the facility near-infrared camera NIRC2 with laser guide star adaptive optics (LGS AO; Wizinowich et al. 2006; van Dam et al. 2006). We kept the LGS centered in NIRC2's narrow field-of-view camera while we obtained dithered images of the target in the $Y_{\mathrm{NIRC} 2}, J_{\mathrm{MKO}}, \mathrm{H}_{\mathrm{MKO}}$, and $\mathrm{CH}_{4} \mathrm{~s}$ bandpassess. The wavefront sensor recorded flux from the LGS equivalent to a $V \approx$ 9.6-9.8 mag star. The primary star HIP 6407 was used as the tiptilt reference star, and the lower bandwidth sensor monitoring 

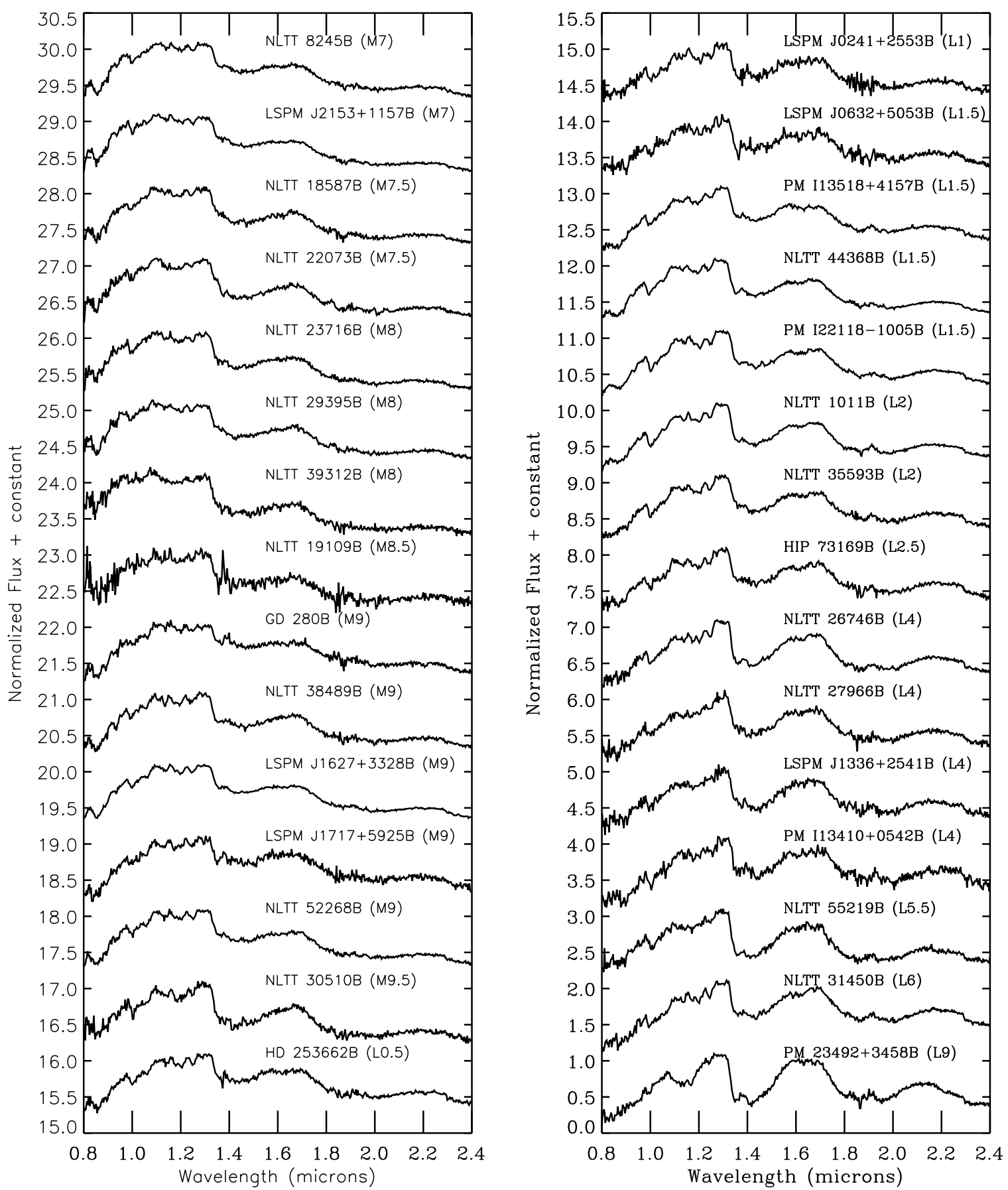

Figure 4. IRTF/SpeX spectra for our ultracool companions discovered serendipitously or by searching for wide companions to faint non-Hipparcos primaries.

this source recorded flux equivalent to a $R \approx 7.6 \mathrm{mag}$ star. Our procedure for reducing and analyzing Keck LGS data is described in detail in our previous work (e.g., Liu et al. 2006; Dupuy et al. 2010). To summarize briefly, we measure binary parameters by fitting three-component Gaussians to determine the position and flux of each binary component, and we derive uncertainties by computing the scatter among individual dithered images. We used the NIRC2 astrometric calibration from Yelda et al. (2010), which includes a correction for the nonlinear distortion of the camera and has a pixel scale of $9.952 \pm 0.002$ mas per pixel and an orientation for the detector's $+y$ axis of $+0.252 \pm 0.009$ east of north. We resolved HIP 6407B as a 0'.131 (7.4 AU) binary. Table 13 gives the binary parameters we measured in each bandpass along with the weighted average of the separation $(\rho)$ and position angle (P.A.) values. Given the objects' separation and maximum total mass of $0.16 M_{\odot}$ (as it consists of two substellar objects), the likely period of this system is $>300 \mathrm{yr}$, making it a poor mass-age benchmark.

We performed spectral decomposition analysis on HIP 6407B using the method described in Section 5.2 of Dupuy \& Liu (2012). Briefly, we started with all possible pairs of the 178 IRTF/SpeX prism spectra from the library of Burgasser et al. (2010). For each template pairing, we determined the scale factors needed to minimize the rms deviation from our observed spectrum. We then computed the $\chi^{2}$ of our Keck LGS AO 

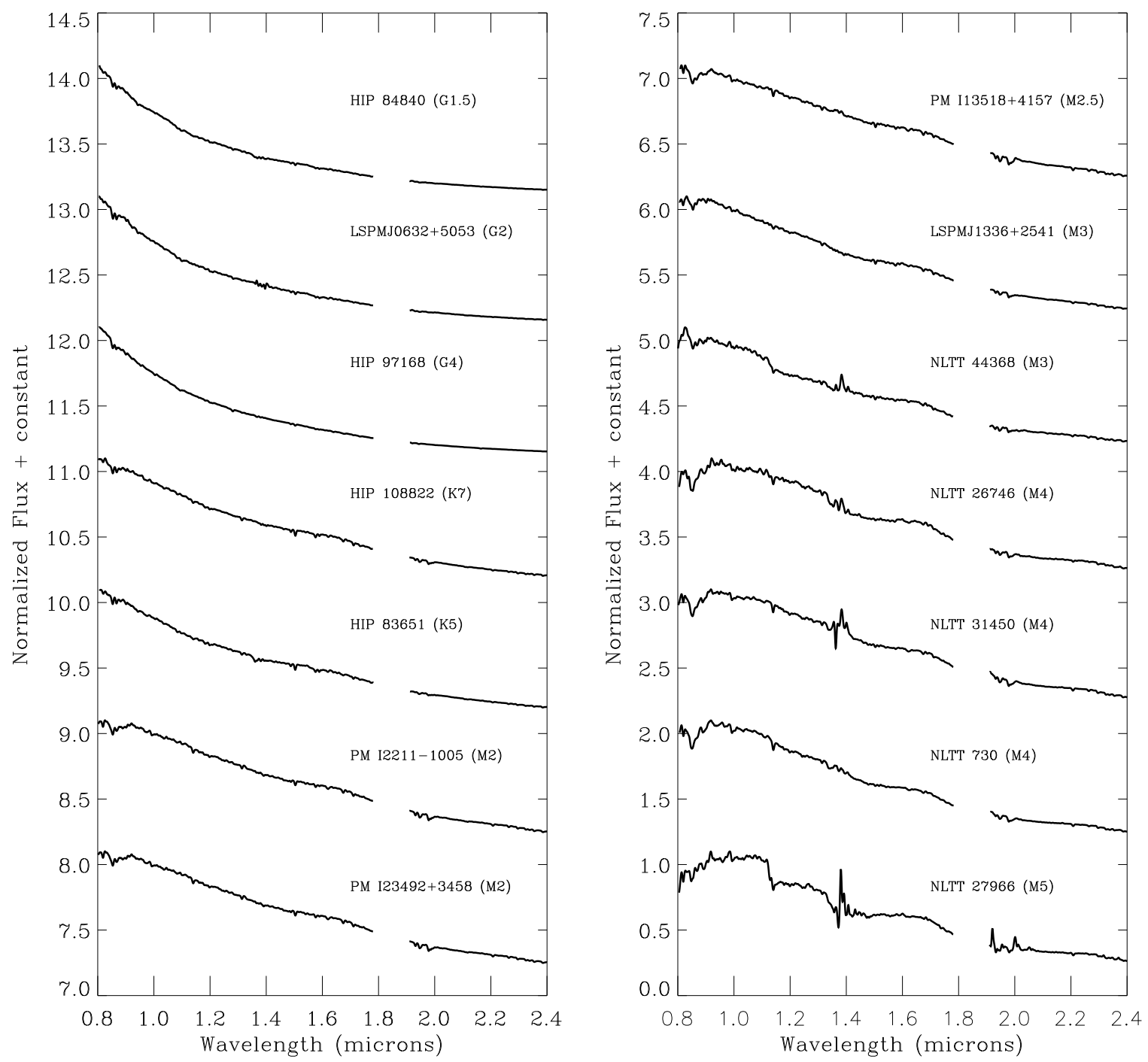

Figure 5. IRTF/SpeX spectra for the primaries of our companions that had no spectral type in the literature. These spectra were taken with SpeX SXD mode and have been Gaussian smoothed to $R=200$.

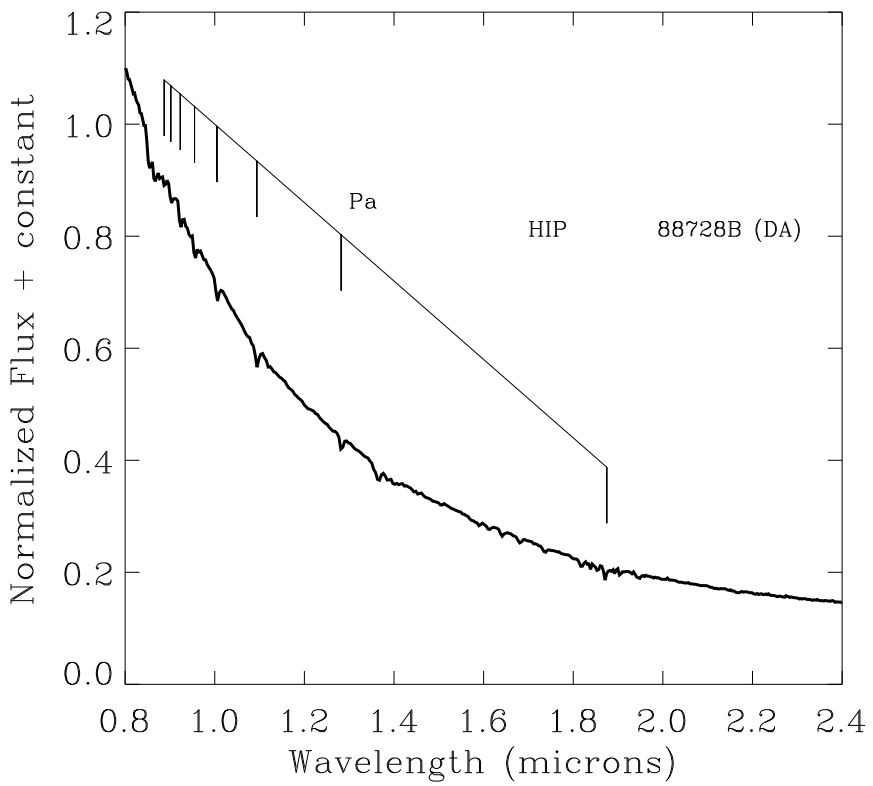

Figure 6. IRTF/SpeX spectra for our white dwarf Hipparcos companions. The wavelengths of the Paschen lines are also shown. flux ratios in $J, H$, and $\mathrm{CH}_{4} \mathrm{~S}$ bands compared to the flux ratios computed for each pairing. We excluded pairings that significantly disagreed with our measured flux ratios, $p\left(\chi^{2}\right)<$ 0.05 , and examined the remaining best pairings to determine the component spectral types. Our final spectral types of L1.0 \pm 0.5 and $\mathrm{T} 3 \pm 1$ account for the full range of spectral templates that gave equally good fits to our combined light spectrum. We estimated the flux ratio in the $K$ band as well as flux ratios in the 2MASS photometric system by taking the mean and rms of each flux ratio among the ensemble of best-fit template pairings. Figure 15 shows the best match to our spectrum, which is provided by the templates.

The best match to our spectrum is provided by the templates DENIS-P J170548.3-051645 (L1; Burgasser et al. 2010; Allers \& Liu 2013) and SDSS J120602.51+281328.7 (T3; Chiu et al. 2006; Burgasser et al. 2010) scaled to each other by the magnitudes shown in the lower panel of Figure 15, giving magnitude differences of $d_{J}=2.26 \pm 0.05 \mathrm{mag}$ and $d_{H}=$ $2.51 \pm 0.05$ mag. We adopt spectral types of $\mathrm{L} 1 \pm 1$ and $\mathrm{T} 3 \pm 1$ for the components of HIP 6407B based on the typical uncertainty in infrared types for L dwarfs and the range of good matching templates for the secondary. Note that the template library from Burgasser et al. (2010) nominally only includes 


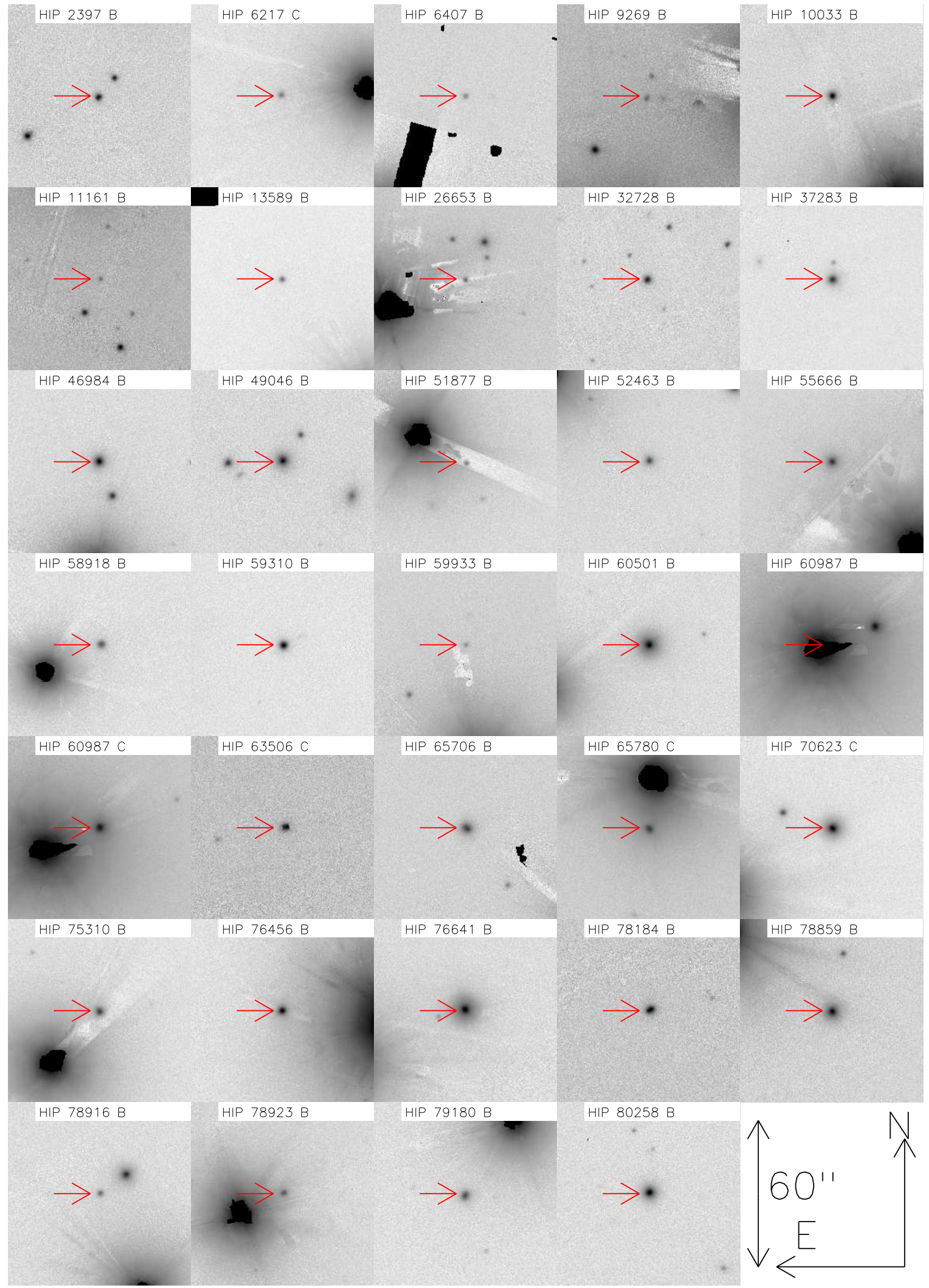

Figure 7. $y_{P 1}$ finder charts for our companions. Note that some regions are masked due to chip gaps and PS1 detector artifacts. Also note that some of the higher proper motion stars appear elongated due to these images being stacks of individual observations spread over the PS1 survey period.

(A color version of this figure is available in the online journal.) 


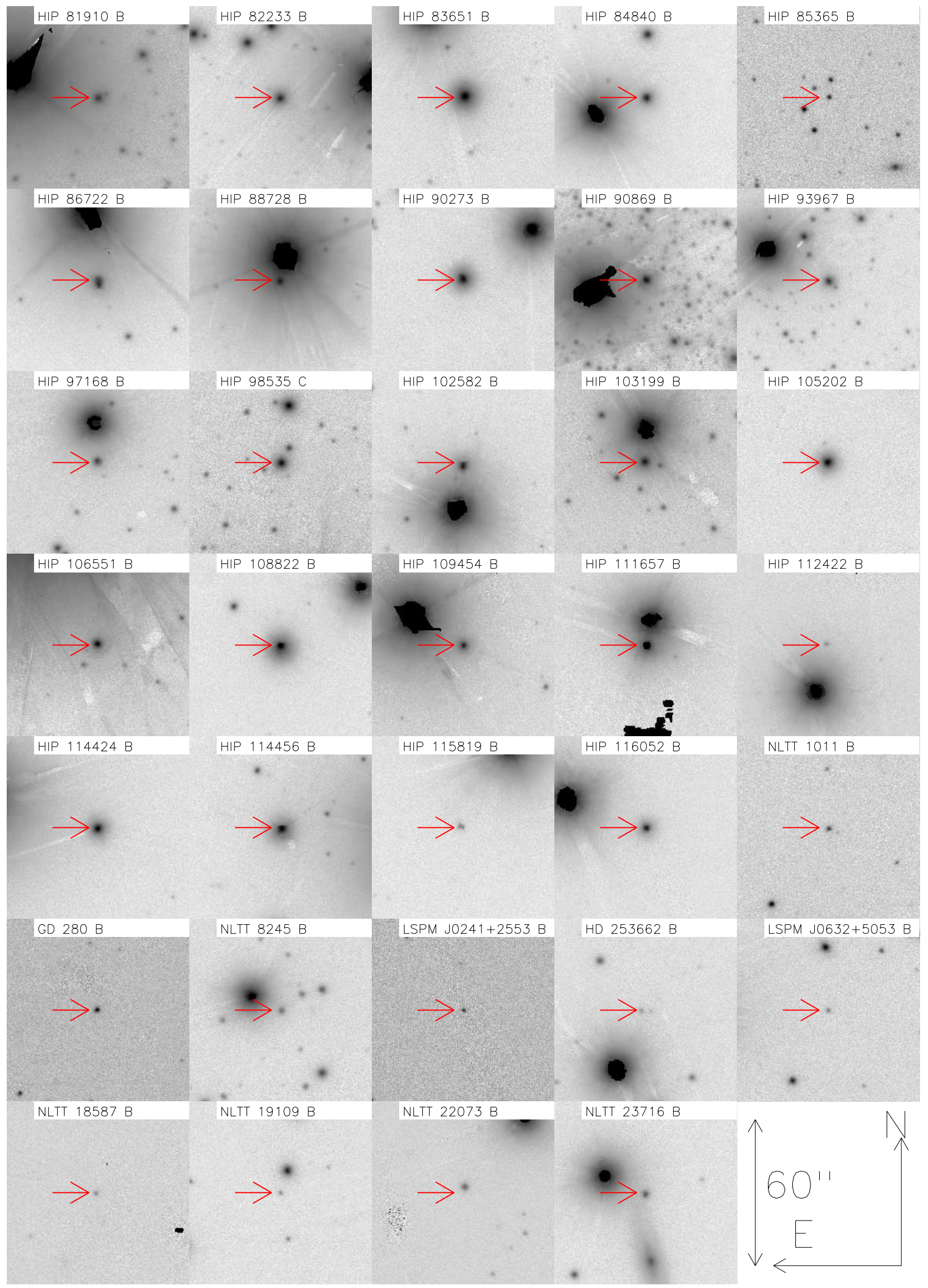

Figure 7. (Continued) 


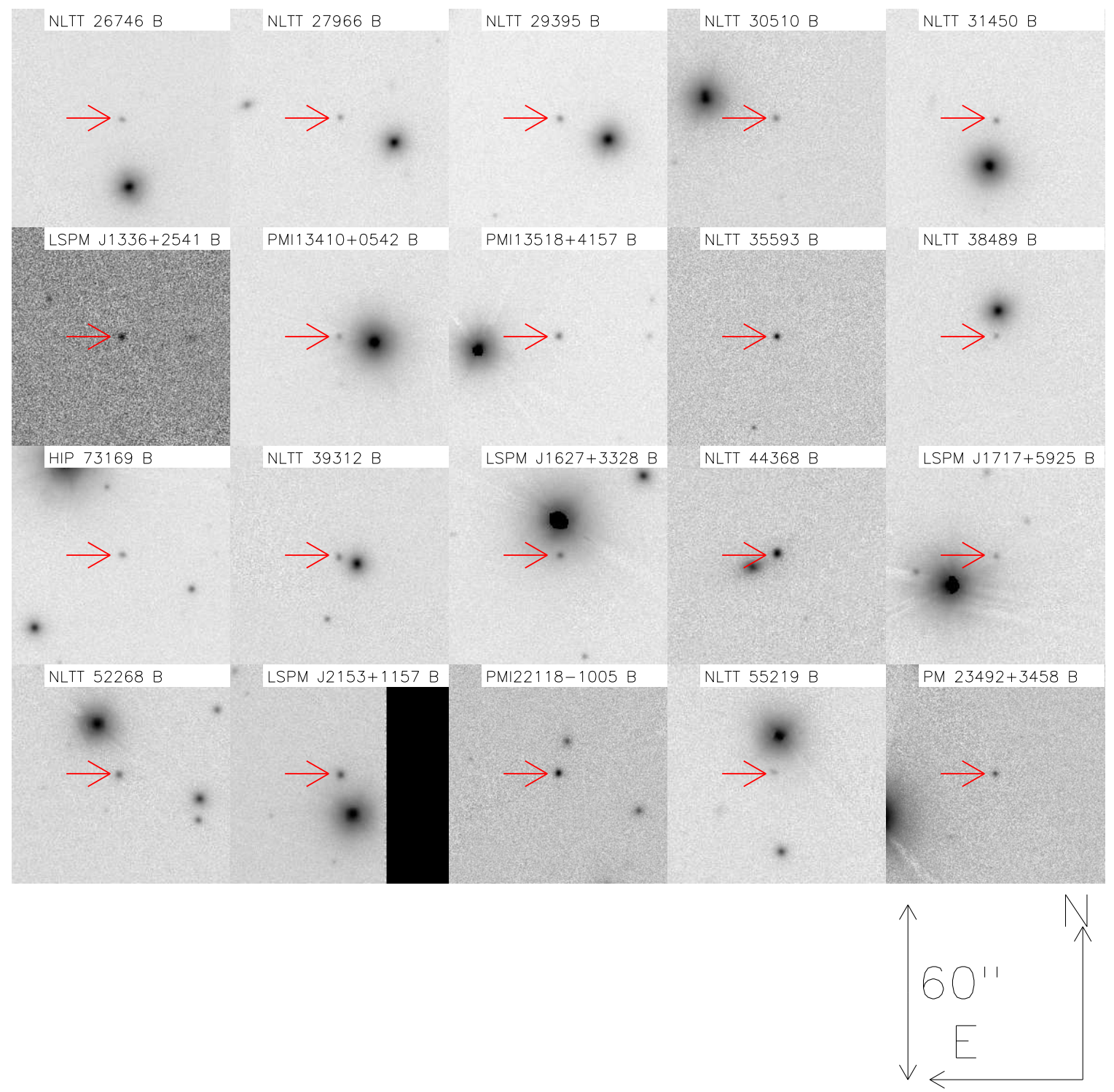

Figure 7. (Continued)

objects later than L0, which barely encompasses the spectral type of the proposed primary component. However, Figure 15 shows that the L1 template gives a very good match to the data, so the primary is not likely to be of much earlier type.

\subsubsection{HIP 70623 (HD 126614)}

HIP 70623 (HD 126614) is a K0 star with an $M \sin i=$ $0.38 M_{\text {Jup }}$ giant planet in a $3.41 \mathrm{yr}$ orbit (Howard et al. 2010). There is also an additional $\mathrm{M}$ dwarf component in the system with a separation of 0.'5 (Howard et al. 2010) detected by adaptive optics imaging. The wide M5.5 companion we recover was identified by Gould \& Chaname (2004) as a companion but has no previously published spectral type. Despite this object being identified as a companion before the Howard et al. (2010) identification of the closer companion, it has the designation HIP 70623/HD 126614 C.

\subsubsection{HIP 115819 (VZ Piscium)}

VZ Piscium is a marginal-contact eclipsing binary with a period of 0.261 days (Hrivnak et al. 1995) classified as a
W-UMa type variable. As angular momentum transfer to additional components can tighten an inner binary and lead to contact, Rucinski et al. (2007) observed VZ Piscium as part of an adaptive optics search for companions to contact binaries. We have identified an M8 companion at a separation of 30". This fell outside the $36^{\prime \prime} \times 36^{\prime \prime}$ field of view of Rucinski et al. (2007). Note that Qian et al. (2004) suggest a closer companion to the binary may be causing a variation in the light curve with a period of $\sim 25$ yr. However, no close companion was identified by Rucinski et al. (2007), and our companion is too wide to introduce such a short period variation.

\subsubsection{HIP 103199 (HD 335289)}

HIP 103199 (spectral type G5) is listed as runaway star by Tetzlaff et al. (2011). This is based on an estimated age of $\sim 46 \pm 23 \mathrm{Myr}$ and the star's space motion. Fujii \& Portegies Zwart (2011) hypothesize that such objects are the result of a three-body interaction that results in the formation of a runaway star and a close binary. HIP 103199 has a wide ( $\geqslant 646$ AU) binary companion found by Lépine \& Bongiorno 2007, which we classify as M3.5. We converted our UKIRT photometry 

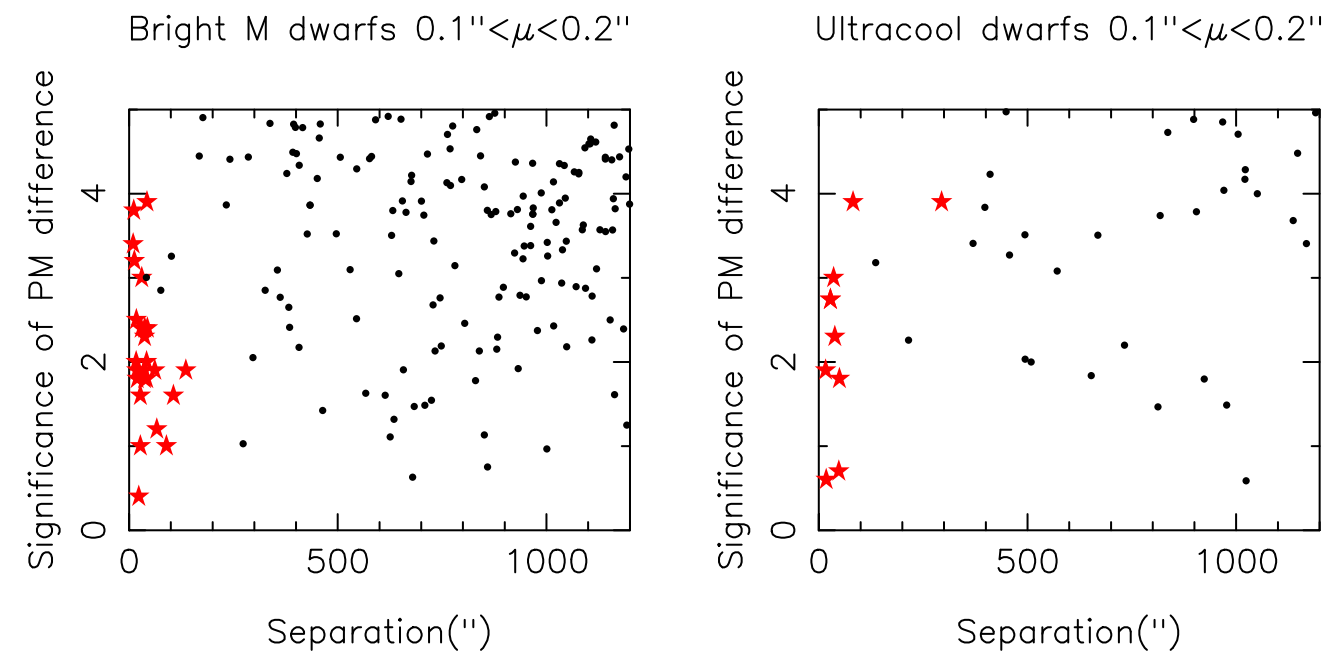

Bright M dwarfs $0.2^{\prime \prime}<\mu<0.3^{\prime \prime}$

Ultracool dwarfs $0.2^{\prime \prime}<\mu<0.3^{\prime \prime}$
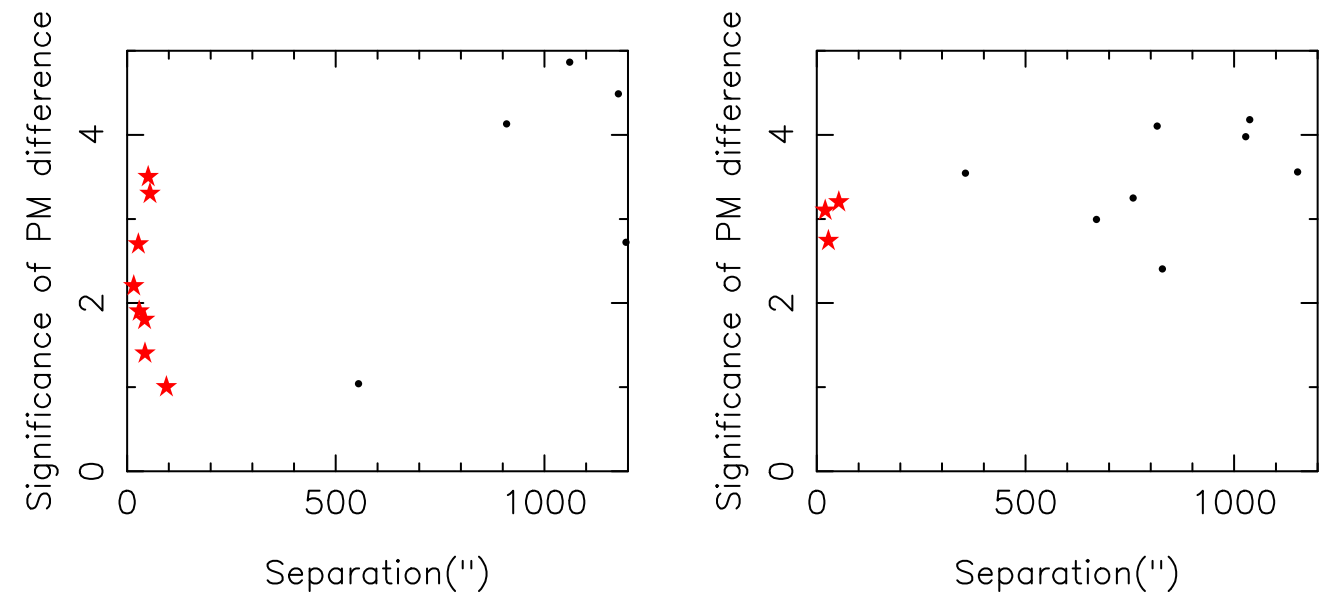

Bright M dwarfs $\mu>0.3^{\prime \prime}$

Ultracool dwarfs $\mu>0.3^{\prime \prime}$
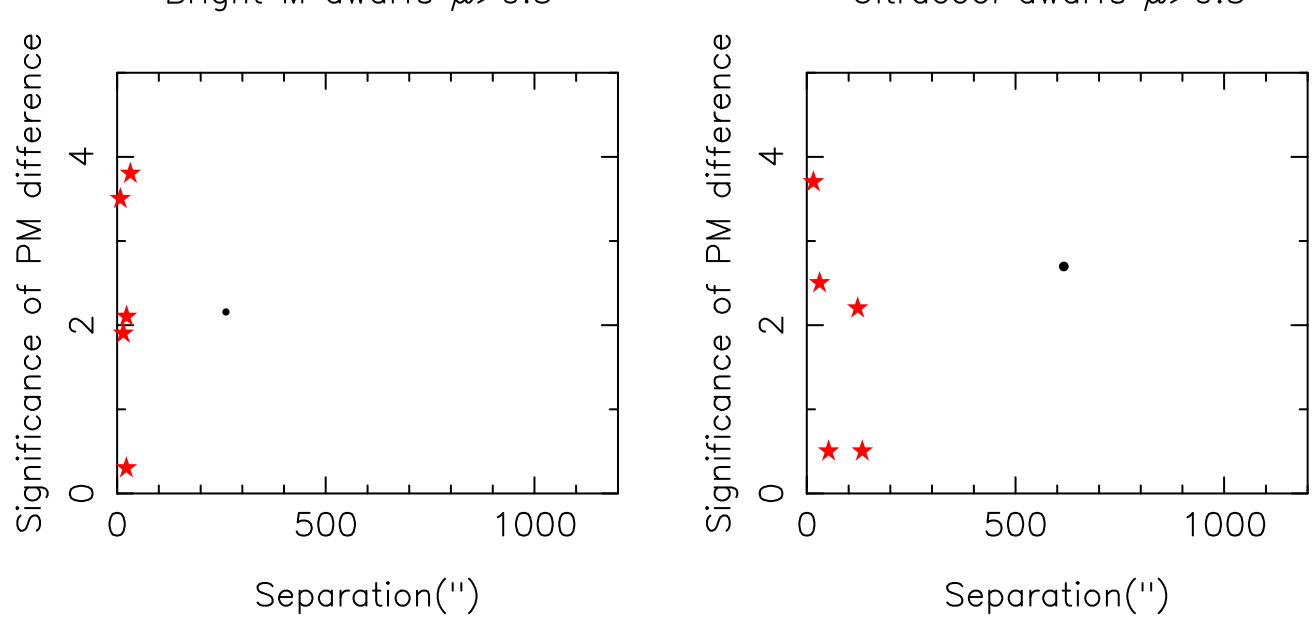

Figure 8. Our bright M (left column) and ultracool (right column) dwarf companions to Hipparcos stars (red stars) compared to a population of coincident objects (black dots). The coincident objects were generated using a method similar to that of Lépine \& Bongiorno (2007) by offsetting the positions of primary stars in our input file and then searching for companions around these positions. The significance of the proper motion difference is the quadrature sum of the proper motion difference in each axis divided by the total proper motion error on that axis (see Equation (1)). Both of our samples lie in areas of the plot that are sparsely populated by coincident pairings.

(A color version of this figure is available in the online journal.) 

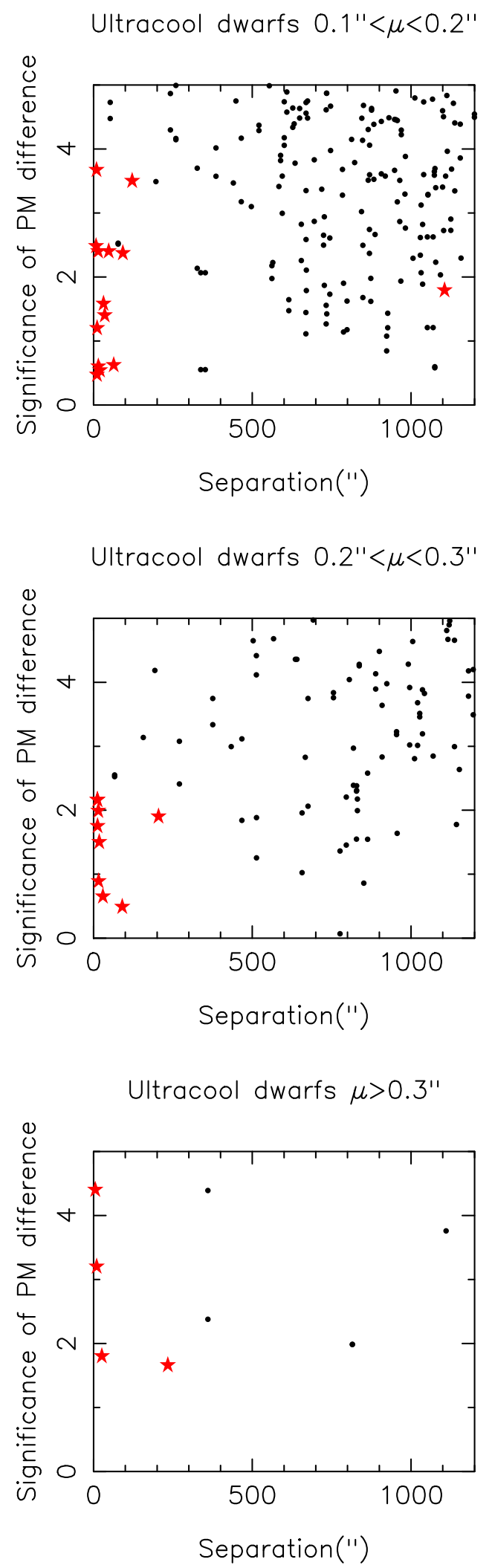

Figure 9. Our ultracool dwarf companions to faint non-HIP primaries (denoted by red stars) compared to a coincident population of objects (black dots). See Figure 8 caption for more details on the process. It appears one object, the apparent companion NLTT $35593\left(\mu=0^{\prime \prime} 19 \mathrm{yr}^{-1}\right)$, lies in a region inhabited by many coincident pairings. Hence, despite the pair's similar photometric distances, we consider this to be an unlikely companion, i.e., one which should be confirmed through other means.

(A color version of this figure is available in the online journal.)

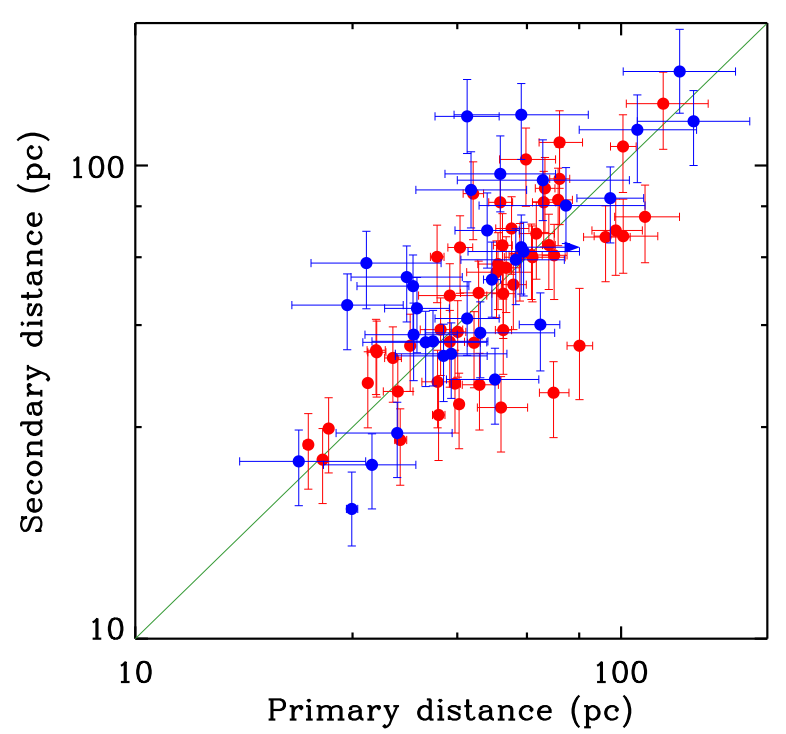

Figure 10. Plot of the photometric distances to our secondaries compared to the distances to the primaries. Points plotted in red have trigonometric parallaxes for their primaries, those in blue have photometric distance estimates. Note that one of our objects (HD 253662) is a known subgiant and hence our quoted photometric distance is a lower limit.

(A color version of this figure is available in the online journal.)

to the CIT system using the conversions of Carpenter (2001) in order to calculate the properties of the companion using evolutionary models. Using the Hipparcos distance to the primary of $59.5_{-43}^{+5.1} \mathrm{pc}$, we derive an absolute $K$-band magnitude of $7.42 \pm 0.18 \mathrm{mag}$ for the companion. Using the evolutionary models of Baraffe et al. (1998) and the spectral-type-effective temperature scale of Kenyon \& Hartmann (1995), we derive $K$-band absolute magnitudes of $6.18 \pm 0.25 \mathrm{mag}$ at $50 \mathrm{Myr}$ and $7.16 \pm 0.17 \mathrm{mag}$ at $300 \mathrm{Myr}$ for an M3.5 where the quoted error is calculated from our half-subtype classification uncertainty. Based on this we suggest that the companion to HIP 103199 (HD 335289) is not overluminous due to youth, and it is likely that this system is not as young as suggested by Tetzlaff et al. (2011).

\subsubsection{HIP 60987 B/C}

During the spectroscopic observations of our companion to HIP 60987 (spectral type M3, separation 19"), we identified that the primary itself was a visual double. A spectrum of this other companion (spectral type $\mathrm{K} 7$, separation $\sim 5^{\prime \prime}$ ) was also obtained. As both companions had previous listings in the Washington Double Star catalog, we used the previously existing designations for the companions.

\subsubsection{NLTT $38489 A / B$}

The companion to NLTT 38489 stands out as having a photometric distance that is in particularly poor agreement with its primary. However, this object is only 6.'7 away from its primary. Hence the photometry used to estimate the companion's photometric distance may be unreliable.

\subsubsection{LSPM J0241+2553A/B}

We do not have a spectrum for LSPM J0241+2553 A. To characterize this object we examined its reduced proper motion diagram placement compared to Figure 4 of Limoges et al. (2013) and concluded that this object was likely a white dwarf. 

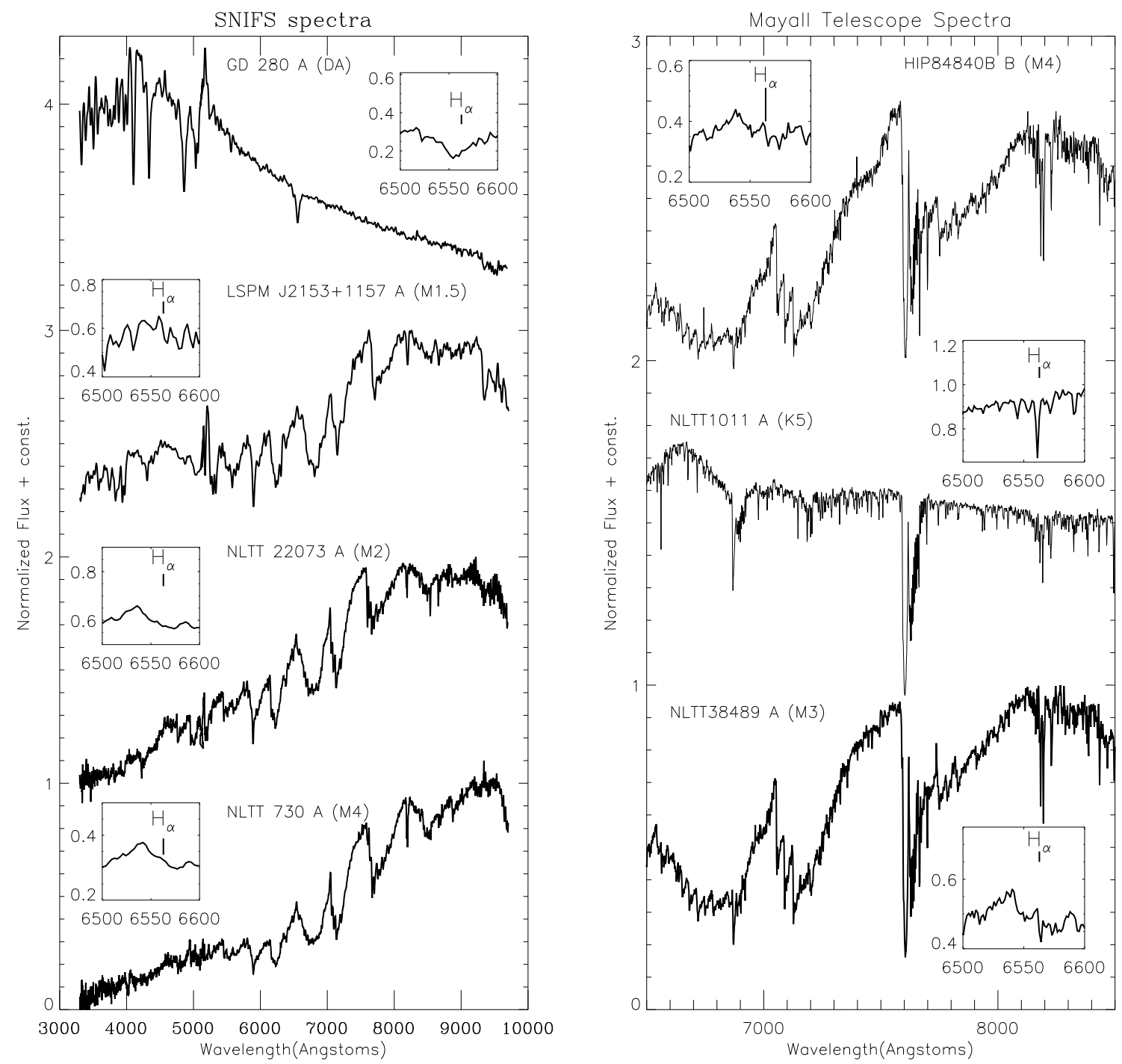

Figure 11. Optical spectroscopy of our primary stars and one companion. Left: spectra taken with SNIFS on the University of Hawaii 88 inch telescope on Mauna Kea. Right: spectra taken with the Ritchey-Chretien Spectrograph on the Mayall $4 \mathrm{~m}$ telescope on Kitt Peak. The spectra for GD 280 A and LSPM J2153+1157 A were both noisy and so have been Gaussian smoothed to $R=300$ to make their spectral features clearer. Note that the feature in the SNIFS spectra at $5200 \AA \AA$ is due to the boundary between the SNIFS red and blue channels. Note that the 7490-7700 A region is strongly affected by tellurics.

\subsection{8. $H D 253662 A / B$}

HD $253662 \mathrm{~A}$ is listed in SIMBAD as a G8 subgiant. We estimated this object's photometric distance using the same mechanism as for other photometric distances using the G8 spectral type, the object's 2MASS magnitudes, and the absolute magnitudes quoted in Kraus \& Hillenbrand (2007). As this object is a subgiant and hence more luminous than the dwarfs used to calibrate the Kraus \& Hillenbrand (2007) absolute magnitudes, we quote our $1 \sigma$ lower error bound as a minimum distance.

\subsubsection{The Possible Companion to NLTT 35593}

The candidate companion to NLTT 35593 has a very similar photometric distance $\left(65.8 \pm_{10.7}^{12.8} \mathrm{pc}\right)$ to its primary $\left(63.0 \pm_{14.6}^{19.1} \mathrm{pc}\right)$. However, its wide separation $\left(1106^{\prime \prime}\right)$ puts it in a region similar to that in Figure 9 that is populated by chance alignments. Hence we consider it unlikely to be a bona-fide companion. Radial velocity or parallax measurements will be required to assess whether it is a true binary.

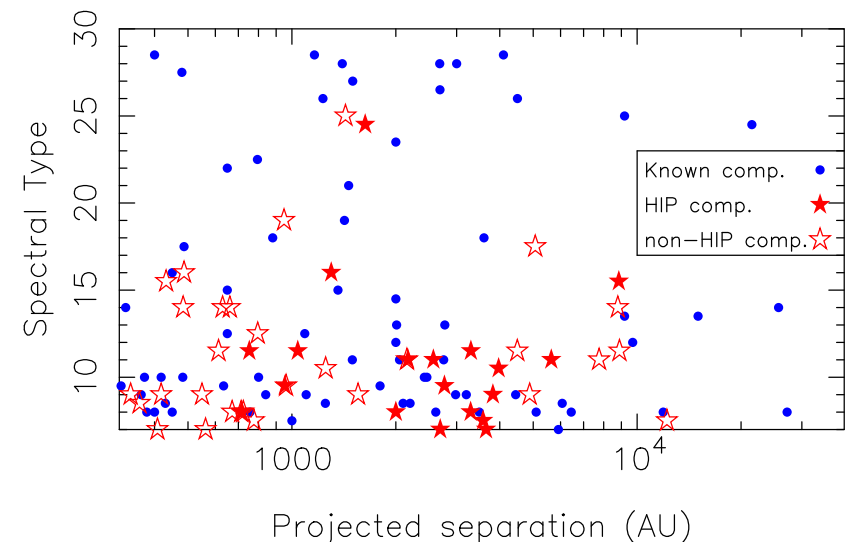

Figure 12. Field ultracool binary population (secondary component $\mathrm{M} 7$ or later). The $y$-axis shows the ultracool companion spectral type, which is 0 at M0, 10 at $\mathrm{L} 0$, and 20 at $\mathrm{T} 0$. The blue dots represent previously identified objects, solid red stars are companions from our Hipparcos search, and open red stars are our companions from other sources. Our two T dwarf discoveries from Deacon et al. (2012b) (a Hipparcos companion) and Deacon et al. (2012a) (a serendipitous companion discovery) are also plotted here.

(A color version of this figure is available in the online journal.) 
Table 10

Data on Our Hipparcos Stars

\begin{tabular}{|c|c|c|c|c|c|c|c|c|c|c|c|}
\hline Object & $\mathrm{SpT}$ & $\begin{array}{c}\mathrm{RV} \\
\left(\mathrm{km} \mathrm{s}^{-1}\right)\end{array}$ & $\begin{array}{c}H_{p} \\
(\mathrm{mag})\end{array}$ & $\begin{array}{l}B-V \\
(\mathrm{mag})\end{array}$ & $\begin{array}{c}{[\mathrm{M} / \mathrm{H}]^{\mathrm{k}}} \\
(\mathrm{dex})\end{array}$ & $\begin{array}{c}{[\mathrm{Fe} / \mathrm{H}]^{\mathrm{e}}} \\
(\mathrm{dex})\end{array}$ & $\begin{array}{l}\text { Age } \\
(\mathrm{Gyr})\end{array}$ & $\begin{array}{c}v_{\tan } \\
\left(\mathrm{km} \mathrm{s}^{-1}\right)\end{array}$ & $\begin{array}{c}U \\
\left(\mathrm{~km} \mathrm{~s}^{-1}\right)\end{array}$ & $\begin{array}{c}V \\
\left(\mathrm{~km} \mathrm{~s}^{-1}\right)\end{array}$ & $\begin{array}{c}W \\
\left(\mathrm{~km} \mathrm{~s}^{-1}\right)\end{array}$ \\
\hline HIP 2397 & $K 5^{\mathrm{a}}$ & $\ldots$ & 9.34 & 0.93 & $\ldots$ & $\ldots$ & $0.5-\sim 10^{y}$ & 42.2 & $\ldots$ & $\ldots$ & $\ldots$ \\
\hline HIP 6217 & $\mathrm{~K} 0^{\mathrm{a}}$ & $\ldots$ & 9.116 & 0.754 & $\ldots$ & $\ldots$ & $0.4-\sim 10^{\mathrm{y} *}$ & 58.5 & $\ldots$ & $\ldots$ & $\ldots$ \\
\hline HIP 6407 & $\mathrm{G} 5^{\mathrm{a}}$ & $\ldots$ & 8.74 & 0.64 & $\ldots$ & $-0.084^{\mathrm{u}}$ & $0.5-\sim 10^{\mathrm{y}}$ & 44.9 & $\ldots$ & $\ldots$ & $\ldots$ \\
\hline HIP 9269 & G5 ${ }^{\mathrm{a}}$ & $-35.3 \pm 0.1^{\mathrm{d}}$ & 7.29 & 0.77 & 0.15 & $\ldots$ & $2.2-10.2^{\mathrm{k}}$ & 50.7 & -10.9 & 15.8 & -57.5 \\
\hline HIP 10033 & $\mathrm{~F}^{\mathrm{a}}$ & $-12.9^{f}$ & 7.24 & 0.5 & $\ldots$ & -0.08 & $2.7-3.7^{\mathrm{e}}$ & 34.3 & -35.8 & 6.4 & -4.4 \\
\hline HIP 11161 & $\mathrm{~F}^{\mathrm{a}}$ & $-41.7^{f}$ & 8.03 & 0.42 & $\ldots$ & -0.23 & $0.5-2.4^{\mathrm{e}}$ & 33.2 & -45.1 & -17.8 & -22.2 \\
\hline HIP 13589 & $\mathrm{~F}^{\mathrm{a}}$ & $\ldots$ & 8.8 & 0.66 & $\ldots$ & $\ldots$ & $0.4-\sim 10^{\mathrm{y}}$ & 61.0 & $\ldots$ & $\ldots$ & $\ldots$ \\
\hline \multirow[t]{2}{*}{ HIP 26653} & G5 ${ }^{q}$ & $12.51 \pm 0.45^{\mathrm{r}}$ & 7.987 & 0.764 & & -0.07 & $1.1-9.3^{\mathrm{e} *}$ & 18.9 & 19.0 & -9.1 & -8.3 \\
\hline & $\ldots$ & $\ldots$ & $\ldots$ & $\ldots$ & -0.1 & $\ldots$ & $1.6-13.8^{\mathrm{k}}$ & & & & \\
\hline HIP 32728 & $\mathrm{G} 0^{\mathrm{a}}$ & $\ldots$ & 9.09 & 0.66 & $\ldots$ & $\ldots$ & $0.3-\sim 10^{y}$ & 52.3 & $\ldots$ & $\ldots$ & $\ldots$ \\
\hline HIP 37283 & $\mathrm{~F}^{\mathrm{a}}$ & $7.0^{\mathrm{f}}$ & 7.0 & 0.47 & $\ldots$ & -0.1 & $0.4-2.1^{\mathrm{e}}$ & 21.7 & 17.0 & -6.6 & -13.7 \\
\hline HIP 46984 & $\mathrm{~F}^{\mathrm{a}}$ & $\ldots$ & 8.81 & 0.52 & $\ldots$ & $\ldots$ & $0.4-\sim 10^{\mathrm{y}}$ & 39.3 & $\ldots$ & $\ldots$ & $\ldots$ \\
\hline HIP 49046 & $\mathrm{M} 0^{\mathrm{a}}$ & $\ldots$ & 11.31 & 1.37 & $\ldots$ & $\ldots$ & $0.3-\sim 10^{\mathrm{z}}$ & 19.7 & $\ldots$ & $\ldots$ & $\ldots$ \\
\hline HIP 51877 & $\mathrm{G} 5^{\mathrm{a}}$ & $-46.68 \pm 0.25^{\mathrm{t}}$ & 8.606 & 0.724 & $\ldots$ & $0.22^{\mathrm{u}}$ & $2.4-7.5^{\mathrm{s}}$ & 17.7 & -17.0 & -32.1 & -45.9 \\
\hline HIP 52463 & $\mathrm{G} 4^{\mathrm{a}}$ & $12.9 \pm 0.2^{\mathrm{f}}$ & 8.82 & 0.64 & $\ldots$ & 0.14 & $1.0-4.9^{\mathrm{e}}$ & 38.4 & 37.4 & -2.1 & 15.4 \\
\hline HIP 55666 & $\mathrm{~F} 5^{\mathrm{a}}$ & $-29.3^{f}$ & 7.06 & 0.54 & $\ldots$ & -0.08 & $3.6-5.1^{\mathrm{e}}$ & 29.8 & 5.9 & -3.9 & -41.2 \\
\hline HIP 58918 & $\mathrm{~K} 1^{\mathrm{a}}$ & $\ldots$ & 10.79 & 1.02 & $\ldots$ & $\ldots$ & $0.1-\sim 10^{\mathrm{y}}$ & 78.7 & $\ldots$ & $\ldots$ & $\ldots$ \\
\hline HIP 59310 & $\mathrm{~K} 5^{\mathrm{a}}$ & $\ldots$ & 10.19 & 1.1 & $\ldots$ & $\ldots$ & $0.2-\sim 10^{\mathrm{y}}$ & 34.1 & $\ldots$ & $\ldots$ & $\ldots$ \\
\hline HIP 59933 & $\mathrm{~F}^{\mathrm{a}}$ & $-3.4^{\mathrm{f}}$ & 8.28 & 0.54 & $\ldots$ & 0.03 & $0.3-2.5^{\mathrm{e}}$ & 29.4 & 19.1 & -20.7 & -9.1 \\
\hline HIP 60501 & $\mathrm{M} 0^{\mathrm{a}}$ & $\ldots$ & 10.72 & 1.58 & $\ldots$ & $\ldots$ & $0.3-\sim 10^{\mathrm{z}}$ & 36.1 & $\ldots$ & $\ldots$ & $\ldots$ \\
\hline HIP 60987 & $\mathrm{~F} 2^{\mathrm{a}}$ & $\ldots$ & 7.45 & 0.43 & $\ldots$ & -0.12 & $1.3-2.4^{\mathrm{e}}$ & 37.4 & $\ldots$ & $\ldots$ & \\
\hline HIP 63506 & $\mathrm{M} 0^{\mathrm{a}}$ & $\ldots$ & 11.37 & 1.58 & $\ldots$ & $\ldots$ & $0.3-\sim 10^{\mathrm{z}}$ & 79.9 & $\ldots$ & $\ldots$ & $\ldots$ \\
\hline HIP 65706 & $\mathrm{~K} 7^{\mathrm{b}}$ & $\ldots$ & 11.22 & 1.58 & $\ldots$ & $\ldots$ & $0.3-\sim 10^{\mathrm{z}}$ & 63.6 & $\ldots$ & $\ldots$ & \\
\hline HIP 65780 & $\mathrm{~K} 0^{\mathrm{a}}$ & 8.64 & 0.82 & $\ldots$ & $\ldots$ & $\ldots$ & $0.5-\sim 10^{y}$ & 80.0 & $\ldots$ & $\ldots$ & $\ldots$ \\
\hline HIP 70623 & $\mathrm{~K} 0^{1}$ & $\ldots$ & 8.96 & 0.81 & 0.46 & $\ldots$ & $3.3-6.0^{\mathrm{k}}$ & 73.0 & $\ldots$ & $\ldots$ & $\ldots$ \\
\hline HIP 75310 & $\mathrm{G5}^{\mathrm{a}}$ & $17.3 \pm 3.9^{f}$ & 8.52 & 0.64 & $\ldots$ & -0.22 & $6.3-9.3^{e}$ & 49.5 & -26.6 & -43.2 & -13.4 \\
\hline HIP 76456 & $\mathrm{~F}^{\mathrm{a}}$ & $-15.7^{\mathrm{f}}$ & 6.56 & 0.433 & $\ldots$ & -0.25 & $0.4-2.2^{\mathrm{e}}$ & 18.4 & -21.3 & 11.0 & 3.0 \\
\hline HIP 76641 & G5 ${ }^{a}$ & $\ldots$ & 8.78 & 0.658 & $\ldots$ & $\ldots$ & $0.4-\sim 10^{y}$ & 42.1 & $\ldots$ & $\ldots$ & $\ldots$ \\
\hline HIP 78184 & $\mathrm{M} 0^{\mathrm{a}}$ & $\ldots$ & 10.42 & 1.27 & $\ldots$ & $\ldots$ & $0.3-\sim 10^{\mathrm{z}}$ & 53.3 & $\ldots$ & $\ldots$ & $\ldots$ \\
\hline HIP 78859 & $\mathrm{G} 0^{\mathrm{a}}$ & $\ldots$ & 8.16 & 0.57 & $\ldots$ & $\ldots$ & $0.7-\sim 10^{y}$ & 70.3 & $\ldots$ & $\ldots$ & $\ldots$ \\
\hline HIP 78916 & $\mathrm{G}^{\mathrm{a}}$ & $\ldots$ & 8.86 & 0.599 & $\ldots$ & $\ldots$ & $0.4-\sim 10^{\mathrm{y}}$ & 59.1 & $\ldots$ & $\ldots$ & $\ldots$ \\
\hline HIP 78923 & $\mathrm{G5}^{\mathrm{a}}$ & $1.2 \pm 0.2^{\mathrm{f}}$ & 8.69 & 0.677 & $\ldots$ & $\ldots$ & $0.4-4.0^{\mathrm{e}}$ & 77.2 & 57.7 & -20.5 & 46.9 \\
\hline HIP 79180 & $\mathrm{~K} 7^{\mathrm{a}}$ & $-26.2 \pm 0.5^{\mathrm{h}}$ & 9.38 & 0.74 & $\ldots$ & $\ldots$ & $0.3-\sim 10^{\mathrm{z}}$ & 97.0 & -99.3 & -12.8 & -8.1 \\
\hline HIP 80258 & $K 3^{\mathrm{a}}$ & $\ldots$ & 10.85 & 0.97 & $\ldots$ & $\ldots$ & $0.1-\sim 10^{y}$ & 69.9 & $\ldots$ & $\ldots$ & $\ldots$ \\
\hline \multirow[t]{2}{*}{ HIP 81910} & $\mathrm{G} 2^{\mathrm{a}}$ & $47.9^{\mathrm{f}}$ & 6.86 & 0.67 & 0.16 & 0.19 & $4.0-5.8^{\mathrm{e}}$ & 23.0 & -46.7 & -25.4 & -0.5 \\
\hline & $\ldots$ & $\ldots$ & $\ldots$ & $\ldots$ & $\ldots$ & $\ldots$ & $3.2-5.0^{\mathrm{k}}$ & & & & \\
\hline \multirow[t]{2}{*}{ HIP 82233} & $\mathrm{G} 2^{\mathrm{a}}$ & $57.4^{\mathrm{f}}$ & 7.55 & 0.57 & $\ldots$ & -0.05 & $0.7-4.7^{\mathrm{e}}$ & 23.9 & -62.0 & -2.2 & -4.0 \\
\hline & $\ldots$ & $\ldots$ & $\ldots$ & $\ldots$ & $\ldots$ & $\ldots$ & $3.8-12.0^{\mathrm{w}}$ & & & & \\
\hline HIP 83651 & $\mathrm{~K} 5^{\mathrm{m}}$ & $\ldots$ & 10.6 & 1.21 & $\ldots$ & $\ldots$ & $0.1-\sim 10^{y}$ & 47.5 & $\ldots$ & $\ldots$ & $\ldots$ \\
\hline HIP 84840 & $\mathrm{G} 1.5^{\mathrm{m}}$ & $\ldots$ & 10.0 & 0.80 & $\ldots$ & $\ldots$ & $0.1-\sim 10^{y}$ & 20.3 & $\ldots$ & $\ldots$ & $\ldots$ \\
\hline HIP 85365 & $\mathrm{~F}^{\mathrm{a}}$ & $0.4^{\mathrm{f}}$ & 4.62 & 0.38 & $\ldots$ & -0.09 & $1.6-1.9^{\mathrm{e} *}$ & 14.6 & -1.9 & -12.0 & 8.1 \\
\hline HIP 86722 & $\mathrm{~K} 0^{\mathrm{a}}$ & $26.5^{\mathrm{f}}$ & 7.64 & 0.75 & $\ldots$ & -0.33 & $3.0-11.8^{\mathrm{e} *}$ & 67.8 & -68.1 & -25.8 & 0.3 \\
\hline HIP 88728 & $\mathrm{~F}^{\mathrm{a}}$ & $16.2 \pm 0.5$ & 7.08 & 0.51 & $\ldots$ & $-0.1^{\mathrm{x}}$ & $1.5-\sim 10^{\mathrm{y}}$ & 29.6 & -29.7 & -6.32 & -14.8 \\
\hline HIP 90273 & $\mathrm{~K} 7^{\mathrm{b}}$ & $\ldots$ & 11.87 & 1.51 & $\ldots$ & $\ldots$ & $0.3-\sim 10^{\mathrm{z}}$ & 67.5 & $\ldots$ & $\ldots$ & $\ldots$ \\
\hline \multirow[t]{2}{*}{ HIP 90869} & $\mathrm{G} 2^{\mathrm{a}}$ & $31.0^{f}$ & 8.07 & 0.58 & $\ldots$ & -0.46 & $5.3-10.8^{\mathrm{e}}$ & 50.4 & -38.9 & -41.8 & 15.6 \\
\hline & $\ldots$ & $\ldots$ & $\ldots$ & $\ldots$ & $\ldots$ & $\ldots$ & $1.9-5.7^{\mathrm{k}}$ & & & & \\
\hline HIP 93967 & $\mathrm{F9}^{\mathrm{a}}$ & $\ldots$ & 9.74 & 0.61 & $\ldots$ & $\ldots$ & $0.2-\sim 10^{\mathrm{y}}$ & 180.5 & $\ldots$ & $\ldots$ & $\ldots$ \\
\hline HIP 97168 & $\mathrm{G} 4^{\mathrm{m}}$ & $\ldots$ & 10.51 & 0.88 & $\ldots$ & $\ldots$ & $0.1-\sim 10^{\mathrm{y}}$ & 85.9 & $\ldots$ & $\ldots$ & \\
\hline HIP 98535 & $\mathrm{~F}^{\mathrm{a}}$ & $-4.9^{f}$ & 8.16 & 0.77 & $\ldots$ & -0.02 & $10.6-13.16^{\mathrm{e}}$ & 42.0 & -38.5 & -12.0 & 12.9 \\
\hline HIP 102582 & $\mathrm{~K} 2^{\mathrm{a}}$ & $61.3 \pm 9.8^{\circ}$ & 9.86 & 1.032 & $\ldots$ & $\ldots$ & $0.2-\sim 10^{\mathrm{y}}$ & 88.9 & -74.9 & -4.1 & -77.6 \\
\hline HIP 103199 & $\mathrm{G} 5^{\mathrm{a}}$ & $\ldots$ & 9.82 & 0.84 & $\ldots$ & $\ldots$ & $0.2-\sim 10^{\mathrm{y}}$ & 45.2 & $\ldots$ & $\ldots$ & $\ldots$ \\
\hline HIP 105202 & $\mathrm{~F}^{\mathrm{a}}$ & $-1.1^{\mathrm{f}}$ & 7.38 & 0.49 & $\ldots$ & -0.29 & $0.6-2.9^{\mathrm{e}}$ & 29.6 & 15.9 & -6.6 & -24.2 \\
\hline HIP 106551 & $\mathrm{~K} 3 \mathrm{III}^{\mathrm{a}}$ & $-68.1 \pm 0.1^{\mathrm{i}}$ & 5.04 & 1.09 & $\ldots$ & $\ldots$ & $\leqslant 10^{\mathrm{aa}}$ & 50.3 & -23.4 & 43.3 & -39.9 \\
\hline HIP 108822 & $\mathrm{~K} 7^{\mathrm{m}}$ & $\ldots$ & 12.11 & 0.0 & $\ldots$ & $\ldots$ & $0.3-\sim 10^{\mathrm{z}}$ & 74.8 & $\ldots$ & $\ldots$ & $\ldots$ \\
\hline HIP 109454 & $\mathrm{~F}^{\mathrm{a}}$ & $6.6^{\mathrm{f}}$ & 8.05 & 0.41 & $\ldots$ & -0.19 & $1.4-2.1^{\mathrm{e}}$ & 54.7 & -52.9 & -1.8 & -15.4 \\
\hline HIP 111657 & $\mathrm{~K} 7^{\mathrm{c}}$ & $\cdots$ & 10.69 & 1.27 & $\ldots$ & $\ldots$ & $0.3-\sim 10^{\mathrm{z}}$ & 61.6 & $\ldots$ & $\ldots$ & $\ldots$ \\
\hline HIP 112422 & $\mathrm{~K} 2^{\mathrm{a}}$ & $\ldots$ & 10.33 & 0.94 & $\ldots$ & $\ldots$ & $0.1-\sim 10^{\mathrm{y} *}$ & 46.8 & $\ldots$ & $\ldots$ & $\ldots$ \\
\hline \multirow[t]{2}{*}{ HIP 114424} & $\mathrm{G}^{\mathrm{a}}$ & $1.7^{\mathrm{f}}$ & 7.45 & 0.6 & 0.06 & 0.08 & $0.6-4.5^{\mathrm{e}}$ & 32.3 & 9.1 & -25.3 & -18.0 \\
\hline & $\ldots$ & $\ldots$ & $\ldots$ & $\ldots$ & $\ldots$ & $\ldots$ & $3.7-11.7^{\mathrm{k}}$ & & & & \\
\hline HIP 114456 & $\mathrm{~K} 0^{\mathrm{a}}$ & $\ldots$ & 7.12 & 0.75 & 0.19 & $\ldots$ & $2.1-9.6^{\mathrm{k}}$ & 34.6 & $\ldots$ & $\ldots$ & $\ldots$ \\
\hline HIP 115819 & $\mathrm{~K} 5^{\mathrm{a}}$ & $-4.3 \pm 1.8^{\mathrm{j}}$ & 10.45 & 1.27 & $\ldots$ & $\ldots$ & $0.2-\sim 10^{\mathrm{y}}$ & 148.5 & 146.8 & -7.8 & -21.6 \\
\hline HIP 116052 & G5 ${ }^{\mathrm{a}}$ & $\ldots$ & 9.33 & 0.8 & $\ldots$ & $\ldots$ & $0.3-\sim 10^{y}$ & 44.9 & $\ldots$ & $\ldots$ & $\ldots$ \\
\hline
\end{tabular}


Notes.

a SIMBAD

${ }^{\mathrm{b}}$ Estimated from $V-J$ color and the relations of Lépine \& Gaidos (2011).

${ }^{c}$ Estimated from 2MASS photometry and the SEDs of Kraus \& Hillenbrand (2007).

${ }^{\mathrm{d}}$ Maldonado et al. (2010).

${ }^{\text {e }}$ Casagrande et al. (2011), quoted age is the $16-84$ percentile range.

${ }^{f}$ Nordstrom et al. (2004).

g Evans (1967).

${ }^{\mathrm{h}}$ Latham et al. (2002).

${ }^{\mathrm{i}}$ Massarotti et al. (2008).

j Bilir et al. (2005).

${ }^{\mathrm{k}}$ Valenti \& Fischer (2005).

${ }^{1}$ Howard et al. (2010).

$\mathrm{m}$ This work.

${ }^{\mathrm{n}}$ Latham (2004).

${ }^{\circ}$ Dawson \& De Robertis (2005).

$\mathrm{p}$ Wielen et al. (2000).

${ }^{\mathrm{q}}$ Montes et al. (2001).

${ }^{\mathrm{r}}$ White et al. (2007).

${ }^{\mathrm{s}}$ Isaacson \& Fischer (2010); note the age quoted in the source does not give a range of values. We obtained a range of values by taking the quoted age and applying the 0.25 dex scatter to the age-activity relation found by Mamajek \& Hillenbrand (2008).

${ }^{\mathrm{t}}$ Chubak \& Marcy (2011).

u Robinson et al. (2007).

${ }^{v}$ Ramírez et al. (2009).

${ }^{w}$ Calculated from the Mamajek \& Hillenbrand (2008) Ca H\&K activity to age relation using the activity measurement of Arriagada (2011).

${ }^{x}$ Metallicity from Lee et al. (2011).

y This work; minimum age calculated from limiting X-ray flux and the relations of Mamajek \& Hillenbrand (2008); approximate maximum age from disk-like kinematics.

${ }^{\mathrm{z}}$ This work; minimum age is that from Shkolnik et al. (2009) for objects with no X-ray emission; approximate maximum age from disk-like kinematics.

aa This work; approximate maximum age from disk-like kinematics.

${ }^{\mathrm{bb}}$ Gontcharov (2006).

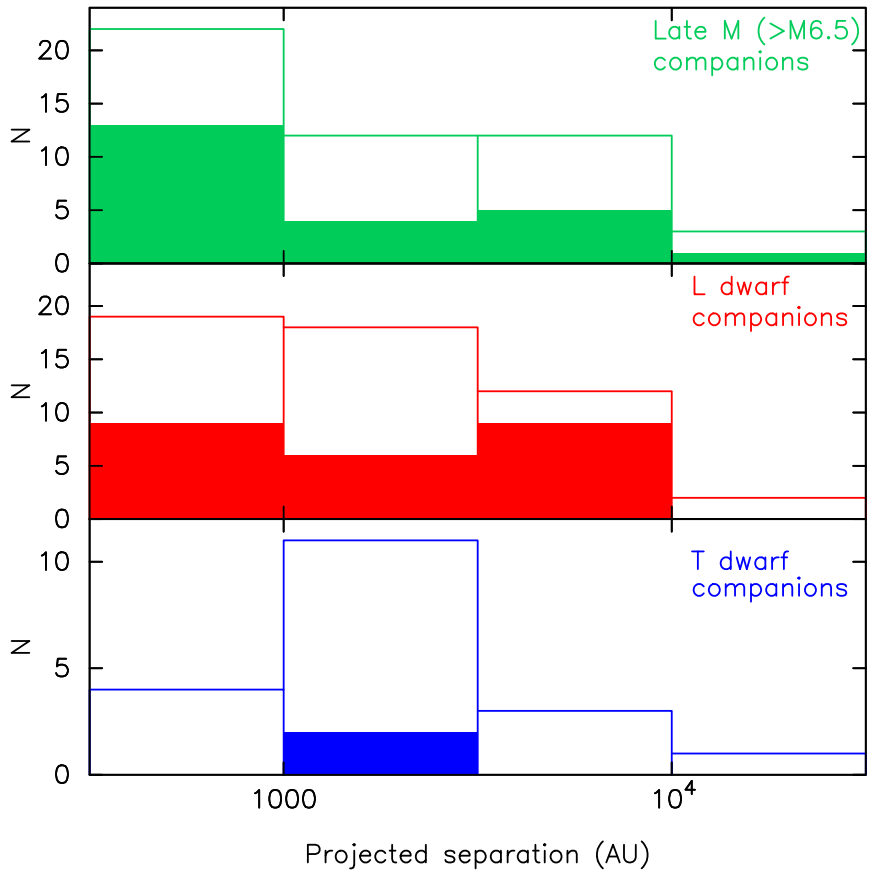

Figure 13. Histograms showing the projected separations of the wide ( $>100 \mathrm{AU})$ companion population. The top panel shows M7, M8, and M9 dwarfs; the middle panel L dwarfs; and the lower panel T dwarfs. For each spectral bin, the open histogram is the total population and the solid histogram is the contribution from our PS1-based efforts (this paper; Deacon et al. 2012a, 2012b).

(A color version of this figure is available in the online journal.)

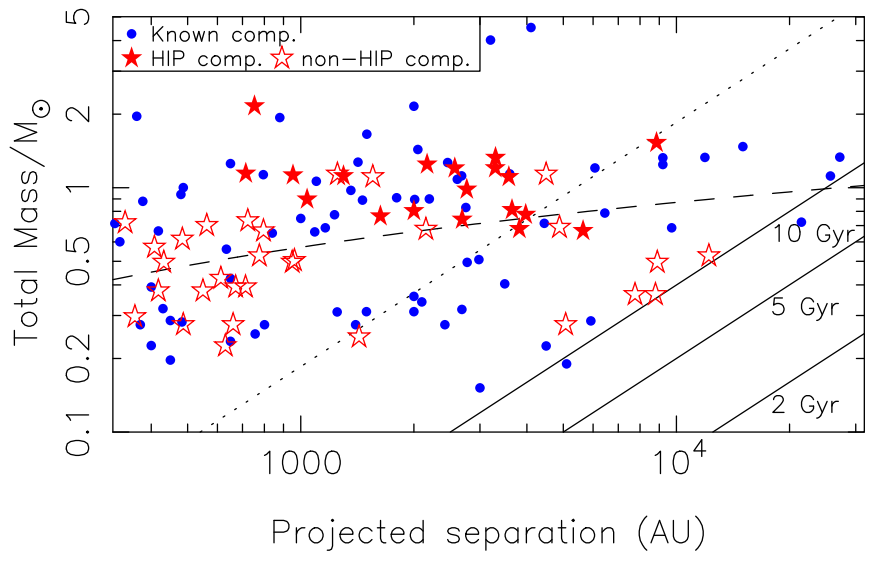

Figure 14. Total mass vs. separation for binary systems with at least one ultracool dwarf component from the literature and from our discoveries. The plot symbols are the same as Figure 12. In cases where we did not have an estimated mass for a substellar companion, we used a mass of $0.075 M_{\odot}$. Hence these are upper limits on the total mass. All other masses are derived from the literature or from the spectral type to mass relation from Kraus \& Hillenbrand (2007). The dotted line represents the approximate maximum separation (equivalent to $v_{\text {esc }}=0.57 \mathrm{~km} \mathrm{~s}^{-1}$ ) suggested by Close et al. (2003) while the dashed line is the suggested log-normal maximum separation suggested by Reid et al. (2001). The three solid lines are the typical separations beyond which a binary is expected to be broken up by interactions in the Galactic disk over the course of 2, 5, and 10 Gyr (Dhital et al. 2010).

(A color version of this figure is available in the online journal.) 
Table 11

Data on Our other Primary Stars

\begin{tabular}{|c|c|c|c|c|c|c|c|c|c|}
\hline Object & SpT & $\begin{array}{c}V \\
\text { (mag.) }\end{array}$ & $\begin{array}{c}V-J \\
\text { (mag.) }\end{array}$ & $\begin{array}{l}\text { Distance } \\
\text { (pc.) }\end{array}$ & $\begin{array}{c}\mu_{\alpha} \cos \delta \\
\left(\text { as } \mathrm{yr}^{-1}\right)\end{array}$ & 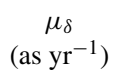 & $\begin{array}{l}\text { Age } \\
\text { (Gyr) }\end{array}$ & $\begin{array}{c}{[\mathrm{M} / \mathrm{H}]} \\
(\mathrm{dex})\end{array}$ & $\begin{array}{c}v_{\tan } \\
\left(\mathrm{km} \mathrm{s}^{-1}\right)\end{array}$ \\
\hline \multicolumn{10}{|c|}{ Other Companion Discoveries } \\
\hline NLTT 1011 & $\mathrm{~K} 5^{\mathrm{b}}$ & 11.13 & 2.0 & $68.2_{-6.5}^{+6.6}$ & -0.076 & -0.192 & $0.3-\sim 10^{\mathrm{i}}$ & $\ldots$ & 66.8 \\
\hline GD 280 & $\mathrm{DA}^{\mathrm{b}}$ & 16.69 & 0.02 & $77_{-26}^{+35}$ & 0.169 & -0.055 & $<10^{\mathrm{k}}$ & $\ldots$ & \\
\hline NLTT 8245 & $\mathrm{M} 0^{\mathrm{c}}$ & 12.63 & 2.98 & $53.0_{-7.5}^{+8.7}$ & -0.093 & -0.164 & $0.3-\sim 10^{\mathrm{i}}$ & $\ldots$ & 47.4 \\
\hline LSPM J0241+2553 & $\mathrm{WD}^{1}$ & 18.19 & 1.19 & $69_{-23}^{+35}$ & -0.037 & -0.153 & $<10^{\mathrm{k}}$ & $\ldots$ & \\
\hline HD 253662 & G8IV $^{\mathrm{d}}$ & 9.92 & 1.36 & $>62.3$ & -0.013 & -0.164 & $0.3-<10^{\mathrm{k}}$ & $\ldots$ & \\
\hline LSPM J0632+5053 & $\mathrm{G} 2^{\mathrm{b}}$ & 9.87 & 1.26 & $95.0_{-13.9}^{+16.3}$ & 0.035 & -0.154 & $0.2-\sim 10^{\mathrm{g}}$ & $\ldots$ & 71.1 \\
\hline NLTT 18587 & $\mathrm{M} 2^{\mathrm{c}}$ & 16.07 & 3.56 & $132_{-31}^{+40}$ & 0.177 & -0.109 & $0.3-\sim 10^{\mathrm{i}}$ & $\ldots$ & 130 \\
\hline NLTT 19109 & $\mathrm{M} 4^{\mathrm{c}}$ & 17.8 & 4.33 & $132_{-36}^{+49}$ & 0.035 & -0.165 & $0.3-\sim 10^{\mathrm{i}}$ & $\ldots$ & 106 \\
\hline NLTT 22073 & $\mathrm{M} 2^{\mathrm{b}}$ & 12.51 & 3.43 & $29.9_{-6.9}^{+12.7}$ & -0.315 & 0.16 & $0.4-\sim 10^{\mathrm{h}}$ & $0.06^{\mathrm{b}}$ & 50.1 \\
\hline NLTT 23716 & $\mathrm{~K} 7^{\mathrm{c}}$ & 11.84 & 2.70 & $48.2_{-6.8}^{+7.9}$ & 0.060 & -0.277 & $0.3-\sim 10^{\mathrm{i}}$ & $\ldots$ & 64.8 \\
\hline NLTT 26746 & $\mathrm{M} 4^{\mathrm{b}}$ & 15.68 & 4.55 & $41.0_{-10.3}^{+12.2}$ & -0.251 & -0.147 & $0.3-\sim 10^{\mathrm{i}}$ & $\ldots$ & 56.5 \\
\hline NLTT 29395 & $\mathrm{M}^{\mathrm{c}}$ & 15.00 & 3.87 & $56.4_{-13.0}^{+16.9}$ & 0.165 & -0.112 & $0.3-\sim 10^{\mathrm{i}}$ & $\ldots$ & 53.3 \\
\hline NLTT 30510 & $\mathrm{M} 2^{\mathrm{c}}$ & 14.17 & 3.66 & $49.1_{-11.3}^{+14.8}$ & 0.218 & -0.390 & $0.3-\sim 10^{\mathrm{i}}$ & $\ldots$ & 104 \\
\hline NLTT 31450 & $\mathrm{M} 4^{\mathrm{b}}$ & 14.93 & 4.15 & $34.6_{-8.7}^{+10.3}$ & -0.034 & -0.202 & $0.3-\sim 10^{\mathrm{i}}$ & $\ldots$ & 33.6 \\
\hline PMI 13410+0542 & $\mathrm{M} 1^{\mathrm{c}}$ & 13.13 & 3.21 & $51.3_{-11.8}^{+21.7}$ & 0.047 & -0.014 & $0.3-\sim 10^{\mathrm{i}}$ & $\ldots$ & 11.9 \\
\hline PMI 13518+4157 & $\mathrm{M} 2.5^{\mathrm{b}}$ & 13.93 & 4.04 & $43.1_{-8.8}^{+9.9}$ & -0.059 & -0.058 & $0.3-\sim 10^{\mathrm{i}}$ & $\ldots$ & 16.9 \\
\hline NLTT 38489 & $\mathrm{M} 3^{\mathrm{b}}$ & 16.05 & 4.22 & $62.3_{-17.0}^{+23.3}$ & -0.147 & 0.183 & $1.2-\sim 10^{\mathrm{h}}$ & $\ldots$ & 69.3 \\
\hline NLTT 39312 & $\mathrm{M} 2^{\mathrm{c}}$ & 16.37 & 3.63 & $141_{-33}^{+43}$ & -0.025 & -0.347 & $0.3-\sim 10^{\mathrm{i}}$ & $\ldots$ & 232.4 \\
\hline LSPM J1627+3328 & $\mathrm{K} 7^{\mathrm{d}}$ & 11.55 & 2.73 & $38.0_{-5.4}^{+6.3}$ & -0.171 & -0.014 & $0.3-\sim 10^{\mathrm{i}}$ & $\ldots$ & 30.9 \\
\hline NLTT 44368 & $\mathrm{M} 3^{\mathrm{b}}$ & 13.17 & 2.78 & $55.0_{-11.2}^{+12.7}$ & -0.056 & 0.215 & $0.3-\sim 10^{\mathrm{i}}$ & $\ldots$ & 57.9 \\
\hline LSPM J1717+5925 & $\mathrm{G} 6^{\mathrm{f}}$ & 10.52 & 1.36 & $108_{-26}^{+35}$ & -0.090 & -0.15 & $0.2-\sim 10^{\mathrm{g}}$ & $\ldots$ & 89.5 \\
\hline NLTT 52268 & $\mathrm{M}^{\mathrm{c}}$ & 14.36 & 3.97 & $37.3_{-8.7}^{+11.3}$ & 0.16 & -0.089 & $0.3-\sim 10^{\mathrm{i}}$ & $\ldots$ & 32.4 \\
\hline LSPM J2153+1157 & $\mathrm{M} 1.5^{\mathrm{b}}$ & 13.9 & 3.82 & $36.2_{-8.4}^{+10.9}$ & -0.093 & -0.128 & $0.3-\sim 10^{\mathrm{i}}$ & $0.26^{\mathrm{b}}$ & 27.1 \\
\hline PM I22118-1005 & $\mathrm{M} 2^{\mathrm{b}}$ & 12.87 & 3.25 & $37.4_{-7.7}^{+8.6}$ & 0.028 & -0.25 & $0.3-\sim 10^{\mathrm{i}}$ & $\ldots$ & 44.6 \\
\hline NLTT 55219 & $\mathrm{M} 2^{\mathrm{c}}$ & 13.93 & 3.65 & $44.7_{-10.4}^{+13.5}$ & 0.324 & 0.16 & $0.3-\sim 10^{\mathrm{i}}$ & $\ldots$ & 76.6 \\
\hline \multicolumn{10}{|c|}{ Serendipitous Companion Discoveries } \\
\hline NLTT 730 & $\mathrm{M} 4^{\mathrm{b}}$ & 15.46 & 4.37 & $21.7_{-5.3}^{+8.1}$ & 0.375 & -0.234 & $3-\sim 10^{\mathrm{i}}$ & $-0.15^{\mathrm{b}}$ & 84.2 \\
\hline NLTT 27966 & $\mathrm{M} 5^{\mathrm{b}}$ & 16.53 & 4.51 & $39.6_{-10.2}^{+13.3}$ & -0.153 & 0.182 & $0.3-\sim 10^{\mathrm{i}}$ & $\ldots$ & 31.7 \\
\hline LSPM J1336+2541 & $M 3^{b}$ & 14.73 & 3.55 & $60.7_{-13.9}^{+15.7}$ & -0.157 & 0.057 & $0.3-\sim 10^{\mathrm{i}}$ & $\ldots$ & 48.1 \\
\hline HIP 73169 & $\mathrm{M} 0^{\mathrm{e}}$ & 11.96 & 3.01 & $27.3_{-6.3}^{+8.3}$ & -0.267 & -0.076 & $0.3-\sim 10^{\mathrm{i}}$ & $\ldots$ & 36.0 \\
\hline PM I23492+3458 & $\mathrm{M} 2^{\mathrm{b}}$ & 12.73 & 3.6 & $30.7_{-6.3}^{+7.1}$ & -0.011 & -0.108 & $0.3-\sim 10^{\mathrm{i}}$ & $\ldots$ & 15.8 \\
\hline \multicolumn{10}{|c|}{ Unlikely Companions } \\
\hline NLTT 35593 & $\mathrm{M} 2^{\mathrm{c}}$ & 14.57 & 3.6 & $63.0_{-14.6}^{+19.1}$ & -0.1837 & -0.0548 & $0.3-\sim 10^{\mathrm{i}}$ & $\ldots$ & 57.3 \\
\hline
\end{tabular}

Notes.

a Approximate spectral type based on 2MASS photometry and the SEDs from Kraus \& Hillenbrand (2007).

$\mathrm{b}$ This work.

${ }^{\mathrm{c}}$ Based on $V-J$ color and the relations presented in Lépine \& Gaidos (2011).

${ }^{\mathrm{d}}$ SIMBAD.

${ }^{\mathrm{e}}$ Gray et al. (2006).

${ }^{\mathrm{f}}$ Hovhannisyan et al. (2009).

g This work; minimum age calculated from limiting X-ray flux and the relations of Mamajek \& Hillenbrand (2008); approximate maximum age from disk-like kinematics.

${ }^{\mathrm{h}}$ This work; minimum age calculated from lack of activity and the activity lifetimes of West et al. (2008); approximate maximum age from disk-like kinematics.

${ }^{i}$ This work; minimum age is that from Shkolnik et al. (2009) for objects with no X-ray emission; approximate maximum age from disk-like kinematics.

j No 2MASS photometry; approximate $V-J$ calculated from optical photometry by Lépine \& Shara (2005).

${ }^{\mathrm{k}}$ This work; approximate maximum age from disk-like kinematics.

${ }^{1}$ This work; based on reduced proper motion diagram placement. 
Table 12

Known Companions with Spectral Type M7 or Later that have Projected Separations of 100 AU or More

\begin{tabular}{|c|c|c|c|c|c|c|c|c|}
\hline \multirow[t]{2}{*}{ Object } & \multirow{2}{*}{$\begin{array}{l}\text { Position } \\
(\mathrm{J} 2000)\end{array}$} & \multicolumn{2}{|c|}{ Separation } & \multirow{2}{*}{$\begin{array}{c}\text { SpT } \\
\text { Companion }\end{array}$} & \multirow{2}{*}{$\begin{array}{c}\text { SpT } \\
\text { Primary }\end{array}$} & \multirow{2}{*}{$\begin{array}{l}\text { Companion Mass } \\
\qquad\left(M_{\odot}\right)\end{array}$} & \multirow{2}{*}{$\begin{array}{l}\text { Age } \\
(\mathrm{Gyr})\end{array}$} & \multirow[t]{2}{*}{ References } \\
\hline & & $(\mathrm{AU})$ & $\left({ }^{\prime \prime}\right)$ & & & & & \\
\hline 2MASS J1258+4013 B & $125837.98+401401.7$ & 6700 & 63 & M7 & M6 & $0.086-0.105$ & $1-5$ & 1 \\
\hline GG Tau Bb & $043230.31+173129.9^{\circ}$ & 210 & 1.5 & M7 & $\mathrm{K} 7+\mathrm{M} 0.5+\mathrm{M} 5$ & $0.038-0.05$ & $0.01-0.02$ & 2 \\
\hline HD $65216 \mathrm{~B}$ & $075342.55-633851.5^{\mathrm{p}}$ & 253 & 7.0 & M7 & G5 & 0.09 & $3-6$ & 3 \\
\hline $\mathrm{C}$ & & & & $\mathrm{L} 2$ & & 0.078 & & \\
\hline$\eta$ Tel B & $192251.26-542530.7$ & 190 & 4.2 & M7/8 & $\mathrm{A} 0 \mathrm{~V}$ & $<0.04$ & $0.0017-0.025^{\mathrm{a}}$ & 4 \\
\hline G121-42 B & $120032.92+204851.3^{q}$ & 5916 & 204.0 & M7 & M4 & $<0.085$ & $4.0-5.0$ & 5 \\
\hline USco 1602-2401 & $160251.17-240150.5$ & 1000 & 7.0 & M7.5 & $\mathrm{K} 4$ & $0.029-0.067$ & $0.010-0.013^{\mathrm{k}}$ & 65 \\
\hline TWA 5 B & $113155.4-343629$ & 100 & 2.0 & M8 & M1.5 & $\sim 0.02$ & $0.01-0.3$ & 6 \\
\hline LP $213-68 \mathrm{Ba}$ & $104712.65+402643.7^{r}$ & 230 & 14 & M8 & M6.5 & $0.080-0.101$ & $\ldots$ & 7,8 \\
\hline $\mathrm{Bb}$ & & & & L1 & & $0.068-0.090$ & & \\
\hline HII 1348 B & $034718.04+242325.73^{\mathrm{t}}$ & 132 & 1.1 & M8 & $\mathrm{K} 5^{*}$ & $0.053-0.055$ & $0.100-0.125$ & 73 \\
\hline $\mathrm{BD}+131727 \mathrm{~B}$ & $073943.86+130507.1$ & 380 & 10.5 & M8+L0.5 & K5 & $\ldots$ & $\ldots$ & 9 \\
\hline V1428 Aql B & $191657.62+050902.2^{r}$ & 400 & 75 & M8 & M3 & $\ldots$ & $\ldots$ & 10 \\
\hline LP $655-23$ B & $043051.58-084900.8$ & 450 & 20 & M8 & M4 & $0.082-0.090$ & $1-8$ & 11,12 \\
\hline 2MASS J0126-5022 B & $012702.83-502321.1$ & 5100 & 82.0 & M8 & M6.5 & $0.062-0.100^{\mathrm{b}}$ & $>0.2^{\mathrm{b}}$ & 13,14 \\
\hline HD 221356 B & $233101.616-040619.39$ & 11900 & 452 & M8 & F8 & 0.088 & $5.5-8$ & 11 \\
\hline $\mathrm{C}$ & & & & L3 & & 0.072 & & \\
\hline HD $221356 \mathrm{D}$ & $233130.95-040523.4$ & 2050 & 12.13 & L1 & $\mathrm{F} 8+\mathrm{M} 8+\mathrm{L} 3$ & $0.073-0.085$ & $2.5-7.9$ & 29 \\
\hline G 266-33 B & $000342.27-282241.0$ & 2610 & 66.0 & M8 & G8 & $0.1-0.103$ & $0.9-1.4$ & 5 \\
\hline G $63-23$ B & $132041.59+095750.6$ & 6445 & 169.0 & M8 & K5 & $0.083-0.093$ & $0.5-3.0$ & 5 \\
\hline NLTT 29131 B & $115824.04-012245.5$ & 3490 & 26.8 & $M 8^{v}$ & M4 & $\ldots$ & $\ldots$ & 77 \\
\hline LSPM J1202+0742 C & $120159.65+073553.6$ & 27200 & 446 & $\mathrm{M}^{\mathrm{v}}$ & $\mathrm{M} 0^{\mathrm{y}}+\mathrm{M} 1^{\mathrm{y}}$ & $\cdots$ & $\cdots$ & 77 \\
\hline SDSS J163126.17+294847.1 B & $163126.17+294836.9^{r}$ & 756 & 10.1 & M8 & M5.5 & $\ldots$ & $\ldots$ & 15 \\
\hline ULAS J132835.49+080819.5 B & $132834.69+080818.9$ & 1250 & 11.9 & M6 & $\mathrm{M} 8.5^{\mathrm{w}}$ & $\ldots$ & $\ldots$ & 77 \\
\hline LSPM J2010+0632 B & $201035.39+063436.7$ & 2100 & 143 & M8.5 & M3.5 & $\ldots$ & $\ldots$ & 16 \\
\hline HD $212168 \mathrm{C}$ & $222644.3-750342$ & 6070 & 265 & M8.5 & G0 & $0.09-0.1$ & $\ldots$ & 17 \\
\hline APMPM J2354-3316 C & $235409.29-331626.6^{r}$ & 2200 & 8.0 & M8.5 & $\mathrm{DA}+\mathrm{M} 4$ & 0.10 & $\sim 1.8$ & 18 \\
\hline USco $1612-1800$ & $161248.97-180049.6$ & 430 & 3.0 & M8.5 & M3 & $0.019-0.042$ & $0.010-0.013^{\mathrm{k}}$ & 65 \\
\hline 2MASS J00301179-3740483 B & $003006.26-373948.3^{r}$ & 89 & 4450 & DA & M9 & $0.07-0.08$ & $>1.9$ & 75 \\
\hline USco1610-1913 B & $161032.33-191308.67$ & 840 & 5.8 & M9 & K7 & $0.017-0.027$ & $0.010-0.013^{\mathrm{k}}$ & 65 \\
\hline HIP $77900 \mathrm{~B}$ & $155430.47-271957.51$ & 3200 & 21.8 & M9 & B6 & $0.017-0.027$ & $0.010-0.013^{\mathrm{k}}$ & 65 \\
\hline GSC 08047-00232 C & $015214.63-521930.0^{\circ}$ & 200 & 3.2 & M9 & $\mathrm{M} 0.5+\mathrm{K} 3$ & $0.015-0.035^{\mathrm{c}}$ & $0.01-0.04$ & 19 \\
\hline NLTT $22980 \mathrm{~B}$ & $095613.13+014514.3^{r}$ & 2980 & 30.4 & M9 & M2 & $\ldots$ & $\ldots$ & 15 \\
\hline HR 6037 B & $161704.35-675626.3$ & 366.0 & 6.7 & M9 & A6 & $0.42-0.82$ & $0.2-0.4$ & 20 \\
\hline SR 12 C & $162719.51-244140.4^{\mathrm{u}}$ & 1100 & 8 & $\mathrm{M}^{\mathrm{m}}$ & $\mathrm{K} 4+\mathrm{M} 2.5$ & $0.012-0.015$ & $\sim 0.002$ & 70 \\
\hline GSC 06214-210 B & $162154.67-204311.3^{t}$ & 320 & 12.9 & M9. $5^{\mathrm{n}}$ & K5 & $0.012-0.016$ & $\sim 0.005$ & 69 \\
\hline $\mathrm{G} 216-7 \mathrm{C}$ & $223732.556+392239.81$ & 634 & 33.6 & M9.5 & M3.5+M3.5 & $0.06-0.08$ & $1-10$ & 21 \\
\hline LEHPM 494 B & $002105.90-424443.3^{\mathrm{r}}$ & 1800 & 78 & M9.5 & M6 & $0.075-0.083$ & $2-10$ & 11 \\
\hline DENIS J0551-4434 B & $055146.05-443411.0^{\circ}$ & 220 & 2.2 & L0 & M8.5 & $\sim 0.06^{\circ}$ & $0.1-10$ & 22 \\
\hline Denis-P J1347-7610 B & $134759.11-761005.4^{\mathrm{r}}$ & 418 & 16.8 & L0 & M0 & $\ldots$ & $0.2-1.4$ & 23 \\
\hline HD 89744 B & $102214.89+411426.7^{r}$ & 2460 & 63 & L0 & F7 & $0.077-0.080$ & $1.5-3$ & 24 \\
\hline NLTT 2274 B & $004154.54+134135.5$ & 483.0 & 23.0 & L0 & M4 & $0.081-0.083$ & $4.5-10.0$ & 5 \\
\hline LP $312-49$ B & $085836.97+271050.8$ & 801 & 15.4 & L0 & M4 & $\ldots$ & $\ldots$ & 15 \\
\hline SDSS J130432.93+090713.7 B & $130433.16+090706.9$ & 374 & 7.6 & L0 & M4.5 & $\ldots$ & $\ldots$ & 15 \\
\hline SDSS J163814.32+321133.5 B & $163817.31+321144.1$ & 2420 & 46.0 & L0 & M4 & $\ldots$ & $\ldots$ & 15 \\
\hline 1RXS J235133.3+312720 B & $235133.48+312722.9^{t}$ & 120 & 2.4 & L0 & M2 & $0.026-0.038$ & $0.05-0.15$ & 71 \\
\hline GJ $1048 \mathrm{~B}$ & $023559.93-233120.5^{\mathrm{r}}$ & 250 & 11.9 & L1 & $\mathrm{K} 2$ & $0.055-0.075$ & $0.6-2$ & 25 \\
\hline AB Pic B & $061912.94-580320.9^{\mathrm{s}}$ & 275 & 5.5 & L1 & $\mathrm{K} 2$ & $\sim 0.01$ & $\sim 0.03^{\mathrm{e}}$ & 26 \\
\hline G124-62 Ba & $144137.167-094559.0^{r}$ & 1496 & 44.0 & L1 & $\mathrm{dM} 4.5 \mathrm{e}$ & $0.054-0.082$ & $0.5-0.8$ & 27 \\
\hline $\mathrm{Bb}$ & & & & $\mathrm{L}^{\mathrm{f}}$ & & $0.054-0.082$ & & \\
\hline GQ Lup B & $154912.09-353903.9^{\mathrm{s}}$ & 103 & 0.7 & L1 & K7 & $0.010-0.020^{\mathrm{g}}$ & $<0.002$ & 28 \\
\hline ROX 42B b & $161031.98-191304.4^{\mathrm{t}}$ & 140 & 1.8 & $\mathrm{~L}^{1}{ }^{\mathrm{m}}$ & M1 & $0.006-0.014$ & $0.0015-0.003$ & 68,79 \\
\hline G $255-24 \mathrm{~B}$ & $133245.31+745944.2$ & 9710 & 38.3 & L2 & K8 & $\ldots$ & $0.2-10.0$ & 30 \\
\hline 2MASS J05254550-7425263 B & $052538.76-742600.8$ & 2000 & 44 & $\mathrm{~L} 2$ & M3 & $0.06-0.075$ & $1.0-10.0$ & 31 \\
\hline G196-3 B & $100420.67+502259.6^{r}$ & 300 & 16.2 & L2 & $\mathrm{M} 2.5$ & $0.015-0.04$ & $0.06-0.3$ & 32 \\
\hline Gl 618.1 B & $162026.147-041631.55$ & 1090 & 35 & $\mathrm{~L} 2.5$ & M0 & $0.06-0.079$ & $0.5-12$ & 24 \\
\hline HD 106906 b & $121752.53-555827.3^{t}$ & 650 & 7.1 & $\mathrm{~L} 2.5$ & F5 & $0.003-0.007$ & $0.013-0.015^{\mathrm{k}}$ & 66 \\
\hline G $63-33 \mathrm{~B}$ & $132044.27+040904.5$ & 2010 & 66 & L3 & $\mathrm{K} 2$ & $0.079-0.081$ & $3.3-5.1$ & 5 \\
\hline G 73-26 B & $020735.60+135556.3$ & 2774 & 73 & L3 & M2 & $0.079-0.081$ & $3.0-4.0$ & $5 / 15$ \\
\hline eta Cancri B & $083231.87+202700.0$ & 15020 & 164 & L3.5 & K3III & $0.063-0.082$ & $2.2-6.1$ & 15 \\
\hline G $171-58 \mathrm{~B}$ & $002503.65+475919.1$ & 9200 & 218 & L4 & F8 & $0.045-0.083$ & $1.8-3.5$ & 5 \\
\hline G $200-28$ B & $141659.78+500626.4$ & 25700 & 570 & L4 & G5 & $0.077-0.078$ & $7.0-12.0$ & 5 \\
\hline LHS 5166 B & $101818.74+590953.7^{r}$ & 160 & 8.43 & $\mathrm{~L} 4$ & M4.5 & $0.055-0.075$ & $2.6-8$ & 27 \\
\hline 1RXS J1609-2105 b & $160930.37-210456.9^{t}$ & 330 & 2.2 & $\mathrm{~L}^{1}$ & M0 & $0.009-0.016$ & $0.010-0.013^{\mathrm{k}}$ & 67 \\
\hline GJ $1001 \mathrm{~B}$ & $000434.85-404405.9$ & 180 & 18.6 & $\mathrm{~L} 4.5$ & M4 & 0.060 .075 & $1-10$ & $33,34,35$ \\
\hline
\end{tabular}




\begin{tabular}{|c|c|c|c|c|c|c|c|c|}
\hline & & & $\begin{array}{r}\text { Table 12 } \\
\text { (Continuec }\end{array}$ & & & & & \\
\hline \multirow[t]{2}{*}{ Object } & \multirow{2}{*}{$\begin{array}{l}\text { Position } \\
\text { (J2000) }\end{array}$} & \multicolumn{2}{|c|}{ Separation } & \multirow{2}{*}{$\begin{array}{c}\text { SpT } \\
\text { Companion }\end{array}$} & \multirow{2}{*}{$\begin{array}{c}\text { SpT } \\
\text { Primary }\end{array}$} & \multirow{2}{*}{$\begin{array}{c}\text { Companion Mass } \\
\left(M_{\odot}\right)\end{array}$} & \multirow{2}{*}{$\begin{array}{l}\text { Age } \\
(\mathrm{Gyr})\end{array}$} & \multirow[t]{2}{*}{ References } \\
\hline & & $(\mathrm{AU})$ & $\left({ }^{\prime \prime}\right)$ & & & & & \\
\hline $\mathrm{C}$ & & & & L4.5 & & 0.060 .075 & & \\
\hline Gl $417 \mathrm{Bab}$ & $111225.674+354813.17^{\mathrm{r}}$ & 2000 & 90.0 & $\mathrm{~L} 4.5+\mathrm{L} 6^{\mathrm{g}}$ & $\mathrm{G} 0+\mathrm{G} 0$ & $0.02-0.05^{\ddagger}$ & $0.08-0.3$ & 34,36 \\
\hline G203-50 B & $171145.59+402857.8^{\mathrm{r}}$ & 135 & 6.4 & L5.0 & M4.5 & $0.051-0.074$ & $1-5$ & 37 \\
\hline GJ 499 B & $130541.07+204639.4$ & 1360 & 516 & L5 & K5+M4 & $\ldots$ & $3.5-10.0$ & 30 \\
\hline G $259-20 \mathrm{~B}$ & $174308.60+852659.4$ & 650 & 30 & L5 & M2.5 & & & 16 \\
\hline LP $261-75$ B & $095105.49+355802.1^{\mathrm{r}}$ & 450 & 13.0 & L6 & M4.5 & $0.019-0.025$ & $0.1-0.2$ & 38 \\
\hline 2MASS J01303563-4445411 B & $013035.80-444541.4$ & 130 & 3.28 & L6 & M9 & $0.032-0.076$ & $0.25-0.8$ & 39 \\
\hline HD 203030 B & $211858.97+261346.1^{\mathrm{s}}$ & 487 & 11.0 & L7.5 & G8 & $0.012-0.031$ & $0.13-0.4$ & 42 \\
\hline Gl 337 CD & $091214.69+145939.69^{r}$ & 43 & $\mathrm{~L} 8+\geqslant \mathrm{L} 8$ & $\mathrm{G} 8+\mathrm{K} 1$ & $0.04-0.074^{\ddagger}$ & $0.6-3.4$ & 24,43 & \\
\hline G1 $584 \mathrm{C}$ & $152322.63+301456.2^{\mathrm{r}}$ & 3600 & 194 & L8 & G1 & $0.045-0.075$ & $1-2.5$ & 44 \\
\hline HD 46588 B & $064627.56+793504.5^{r}$ & 1420 & 79.2 & L9 & F7 & $0.045-0.072$ & $1.3-4.3$ & 45 \\
\hline$\epsilon$ Indi $\mathrm{Ba}$ & $220410.52-564657.7^{r}$ & 1460 & 402.0 & $\mathrm{~T} 1$ & K5 & $0.060-0.073^{\mathrm{i}}$ & $\sim 5^{\mathrm{h}}$ & 46,47 \\
\hline $\mathrm{Bb}$ & & & & T6 & & $0.047-0.060^{\mathrm{h}}$ & $0.5-7.0^{\mathrm{z}}$ & \\
\hline 2MASS J111806.99-064007.8 B & $111807.130-064015.82$ & 650 & 7.7 & $\mathrm{~T} 2$ & M4.5 & $0.06-0.07-6.0$ & $\ldots$ & 48 \\
\hline HN Peg B & $214428.47+144607.8^{r}$ & 795 & 43 & $\mathrm{~T} 2.5$ & G0 & $0.012-0.030$ & $0.1-0.5$ & 49 \\
\hline GU Psc B & $011236.48+170431.8$ & 2000 & 41.97 & $\mathrm{~T} 3.5$ & M3 & $0.07-0.13$ & $0.009-0.013$ & 76 \\
\hline HIP 38939 B & $075801.61-253901.4$ & 1630 & 88 & $\mathrm{~T} 4.5$ & $\mathrm{~K} 4$ & $0.018-0.058$ & $0.3-2.8$ & 50 \\
\hline LSPM J1459+0851 B & $145935.30+085751.6$ & 21500 & 365 & $\mathrm{~T} 4.5$ & $\mathrm{DA}$ & $0.064-0.075$ & $4-10$ & 74 \\
\hline LHS 2803 B & $134802.90-134407.1$ & 1400 & 67.6 & $\mathrm{~T} 5$ & M4.5 & $0.068-0.081$ & $3.5-10$ & 51,31 \\
\hline HD 118865 B & $133943.79+010436.4$ & 9200 & 148 & T5 & F5 & $\ldots$ & $1.5-4.9$ & 52 \\
\hline HIP 73786 B & $150457.66+053800.8$ & 1230 & 63.8 & T6 & K5 & $\ldots$ & $>1.6$ & 53,54 \\
\hline LHS 302 B & $112254.73+255021.5$ & 4500 & 265 & $\mathrm{~T} 6$ & M5 & $\ldots$ & $\ldots$ & 55 \\
\hline G 204-39 B & $175805.46+463309.9^{r}$ & 2685 & 198 & T6.5 & M3 & $0.02-0.035$ & $0.5-3.0$ & 5 \\
\hline Gl 570 D & $145714.96-212147.8^{r}$ & 1500 & 258 & $\mathrm{~T} 7$ & $\mathrm{~K} 4+\mathrm{M} 1.5+\mathrm{M} 3$ & $0.03-0.07$ & $2-5$ & 56 \\
\hline HD 3651 B & $003918.91+211516.8^{r}$ & 480 & 43 & $\mathrm{~T} 7.5$ & $\mathrm{~K} 0$ & $0.018-0.058^{j}$ & $0.7-4.7$ & 49,57 \\
\hline SDSS J1416+30 B & $141623.94+134836.3$ & $45-135$ & 9.0 & $\mathrm{~T} 7.5$ & L6p & $0.03-0.04$ & $\sim 10$ & $58,59,60$ \\
\hline LHS 2907 B & $142320.86+011638.1$ & 2680 & 156 & $\mathrm{~T} 8$ & G1 & $0.019-0.047$ & $2.3-14.4$ & 16,61 \\
\hline LHS $6176 \mathrm{~B}$ & $095047.28+011734.3$ & 1400 & 52 & $\mathrm{~T} 8$ & M4 & $\ldots$ & $>3.5$ & 16,52 \\
\hline Wolf $1130 \mathrm{~B}$ & $200520.38+542433.9$ & 3000 & 188.5 & $\mathrm{~T} 8$ & sd M1.5+DA & $0.020-0.050$ & $>2$ & 72 \\
\hline Ross $458 \mathrm{C}$ & $130041.73+122114.7$ & 1162 & 102 & $\mathrm{~T} 8.5$ & $\mathrm{M} 0.5+\mathrm{M} 7$ & $0.005-0.0014$ & $<1.0$ & 62 \\
\hline$\xi \mathrm{UMa} E$ & $111838.70+312537.9$ & 4100 & 510 & $\mathrm{~T} 8.5$ & $\mathrm{~F} 9+\mathrm{GO}^{\dagger}$ & $0.014-0.038$ & $4.0-8.0$ & 63 \\
\hline Wolf $940 \mathrm{~B}$ & $214638.83-001038.7$ & 400 & 32 & $\mathrm{~T} 8.5$ & M4 & $0.02-0.032$ & $3.5-6$ & 64 \\
\hline WD 0806-661 & $080714.68-661848.7$ & 2500 & 130 & $>\mathrm{YO}$ & DQ & $0.03-0.10$ & $1.2-2$ & 78 \\
\hline
\end{tabular}

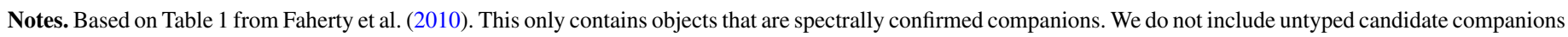
but make an exception for the Y dwarf companion to WD 0806-661 (Luhman et al. 2011), which is too faint in the near-infrared for spectral confirmation.

a Binks \& Jeffries (2014).

b Artigau et al. (2009).

c Chauvin et al. (2005b).

e Mass calculated by Faherty et al. (2010), Faherty private communication.

e Song et al. (2003).

${ }^{\mathrm{f}}$ Reid et al. (2008).

g Burgasser et al. (2005).

${ }^{\mathrm{h}}$ Marois et al. (2007).

${ }^{\mathrm{i}}$ King et al. (2010).

${ }^{\mathrm{j}}$ Liu et al. (2007).

${ }^{k}$ Pecaut et al. (2012).

${ }^{1}$ Lafrenière et al. (2010).

m Bowler et al. (2014).

${ }^{\mathrm{n}}$ Bowler et al. (2011).

${ }^{\circ}$ Position from SIMBAD.

p Position from Washington Double Star Catalogue.

q Position from Webb et al. (1999).

${ }^{\mathrm{r}}$ Position from 2MASS.

${ }^{s}$ Position from Faherty et al. (2009).

${ }^{t}$ Position calculated using the position of the primary and the separation and position angle quoted in the discovery paper.

" Position of primary; discovery paper does not quote a position angle so calculation of the secondary's position is not possible.

$\checkmark$ West et al. (2008).

w Zhang et al. (2010).

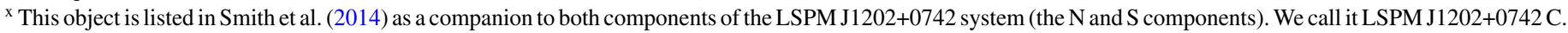

y Spectral type estimated from 2MASS photometry and empirical SEDs for Kraus \& Hillenbrand (2007).

${ }^{\mathrm{z}}$ Liu et al. (2010).

${ }^{\dagger}$ Both of these components of $\xi$ UMa are themselves spectroscopic binaries.

* The primary star is a spectroscopic binary.

$\ddagger$ This mass estimate was based on the spectrophotometric properties of the object before it was determined to be an unresolved binary. 
References. (1) Radigan et al. (2009); (2) White et al. (1999); (3) Mugrauer et al. (2007); (4) Lowrance et al. (2000); (5) Faherty et al. (2010); (6) Lowrance et al. (1999); (7) Gizis et al. (2000); (8) Close et al. (2003); (9) Cruz et al. (2007); (10) van Biesbroeck (1944); (11) Caballero (2007); (12) Cruz et al. (2003); (13) Artigau et al. (2007); (14) Deacon \& Hambly (2007); (15) Zhang et al. (2010); (16) Luhman et al. (2012); (17) Caballero \& Montes (2012); (18) Scholz et al. (2004); (19) Neuhaeuser \& Guenther (2004); (20) Huélamo et al. (2010); (21) Kirkpatrick et al. (2001); (22) Billères et al. (2005); (23) Phan-Bao et al. (2008); (24) Wilson et al. (2001); (25) Gizis et al. (2001); (26) Chauvin et al. (2005a); (27) Seifahrt et al. (2005); (28) Neuhaeuser et al. (2005); (29) Gauza et al. (2012); (30) Gomes et al. (2013); (31) Mužić et al. (2012); (32) Rebolo (1998); (33) Golimowski et al. (2004); (34) Kirkpatrick et al. (1999); (35) Martín (1999); (36) Bouy et al. (2003); (37) Radigan et al. (2008); (38) Reid \& Walkowicz (2006); (39) Dhital et al. (2011); (40) Faherty et al. (2011); (41) Dupuy \& Liu (2012); (42) Metchev \& Hillenbrand (2006); (43) Burgasser et al. (2005); (44) Kirkpatrick et al. (2000); (45) Loutrel et al. (2011); (46) Scholz et al. (2003); (47) McCaughrean et al. (2004); (48) Reylé et al. (2013); (49) Luhman et al. (2007); (50) Deacon et al. (2012b); (51) Deacon et al. (2012a); (52) Burningham et al. (2013); (53) Scholz (2010a); (54) Murray et al. (2011); (55) Kirkpatrick et al. (2011); (56) Burgasser et al. (2000); (57) Mugrauer et al. (2006); (58) Scholz (2010b); (59) Burningham et al. (2010); (60) Bowler et al. (2009); (61) Pinfield et al. (2012); (62) Goldman et al. (2010); (63) Burningham et al. (2013); (64) Burningham et al. (2009); (65) Aller et al. (2013); (66) Bailey et al. (2014); (67) Lafrenière et al. (2008); (68) Kraus et al. (2014); (69) Ireland et al. (2011); (70) Kuzuhara et al. (2011); (71) Bowler et al. (2012); (72) Mace et al. (2013); (73) Geissler et al. (2012); (74) Day-Jones et al. (2011); (75) Day-Jones et al. (2008); (76) Naud et al. (2014); (77) Smith et al. (2014); (78) (Luhman et al. 2011); (79) (Currie et al. 2014).
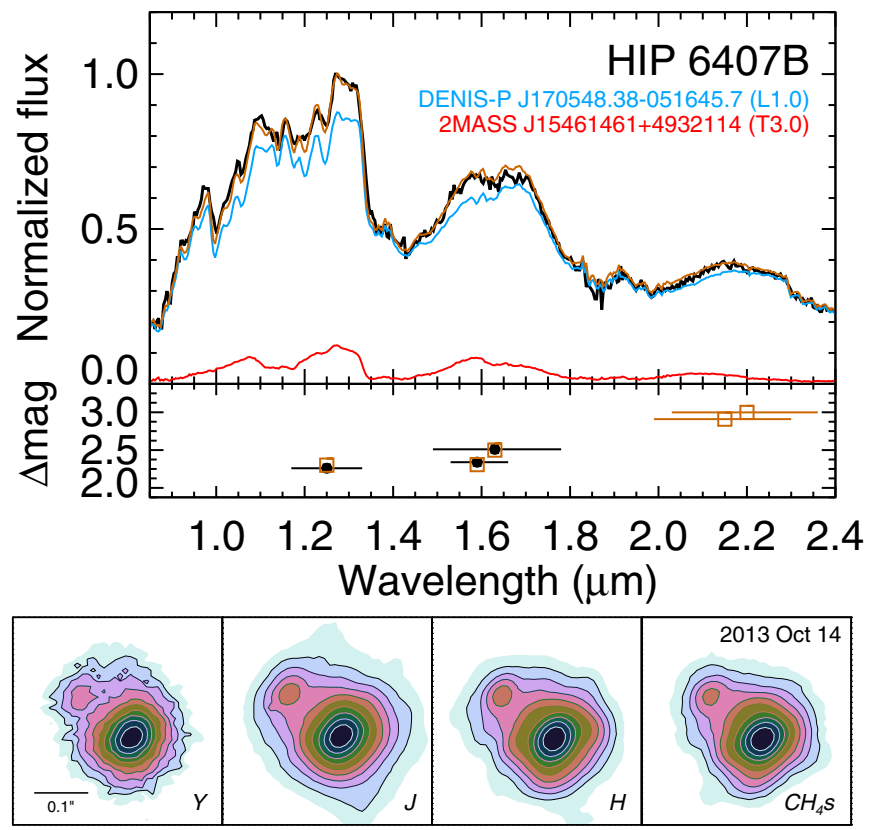

Figure 15. Upper panel: the spectral decomposition of HIP 6407B. The best match was the combination of the L1 dwarf DENIS-P J170548.3-051645 (Burgasser et al. 2010; Allers \& Liu 2013) and the T3 dwarf SDSS J120602.51+281328.7 (Chiu et al. 2006; Burgasser et al. 2010). Middle panel: the resulting flux ratios between the two objects. Lower panel: Keck LGS AO images from which we derive astrometry and flux ratios with contours drawn at logarithmic intervals. Images have been rotated such that north is up.

(A color version of this figure is available in the online journal.)

Table 13

Keck LGS AO Observations of HIP 6407Bab on 2013 October 13 UT

\begin{tabular}{lccc}
\hline \hline Filter & $\begin{array}{c}\rho \\
(\mathrm{mas})\end{array}$ & $\begin{array}{c}\text { P.A. } \\
\left({ }^{\circ}\right)\end{array}$ & $\begin{array}{c}\Delta m \\
(\mathrm{mag})\end{array}$ \\
\hline$Y_{\text {NIRC2 }}$ & $129.7 \pm 2.7$ & $48.4 \pm 1.2$ & $2.13 \pm 0.06$ \\
$J_{\text {MKO }}$ & $125.1 \pm 1.6$ & $51.1 \pm 1.4$ & $2.26 \pm 0.05$ \\
$H_{\text {MKO }}$ & $125.9 \pm 1.0$ & $51.3 \pm 0.4$ & $2.51 \pm 0.05$ \\
$\mathrm{CH}_{4} s$ & $122.4 \pm 2.9$ & $50.3 \pm 1.3$ & $2.34 \pm 0.05$ \\
weighted mean & $125.8 \pm 0.8$ & $51.0 \pm 0.4$ & $\cdots$ \\
\hline
\end{tabular}

\section{CONCLUSIONS}

We have presented 57 newly discovered companions to known nearby stars, with 24 of these being previously unknown L dwarf companions. With the addition of spectral classification of previously known objects, we have characterized a total of 88 wide, common proper motion companions to nearby stars. We have increased the sample of late $\mathrm{M}$ companions with projected separations greater than $\sim 300 \mathrm{AU}$ by $88 \%$ and increased the number of $\mathrm{L}$ dwarf companions in the same separation range by $82 \%$. Examination of our discoveries and the previously known wide ultracool companion population indicates that although many of the systems are loosely bound, they are unlikely to be distrupted over several Gyr. This paper provides a large sample of wide ultracool companions to stars, which are excellent laboratories for testing models of substellar evolution and atmospheres. Additionally our late-type companions provide an opportunity to extend metallicity determinations for $\mathbf{M}$ dwarfs to cooler temperatures (Mann et al. 2014).

The Pan-STARRS1 Surveys (PS1) have been made possible through contributions of the Institute for Astronomy, the University of Hawaii, the Pan-STARRS Project Office, the MaxPlanck Society and its participating institutes, the Max Planck Institute for Astronomy, Heidelberg and the Max Planck Institute for Extraterrestrial Physics, Garching, The Johns Hopkins University, Durham University, the University of Edinburgh, Queen's University Belfast, the Harvard-Smithsonian Center for Astrophysics, the Las Cumbres Observatory Global Telescope Network Incorporated, the National Central University of Taiwan, the Space Telescope Science Institute, the National Aeronautics and Space Administration under grant No. NNX08AR22G issued through the Planetary Science Division of the NASA Science Mission Directorate, the National Science Foundation under grant No. AST-1238877, the University of Maryland, and Eotvos Lorand University (ELTE). The authors thank Bill Golisch, Dave Griep, and Eric Volqardsen for assisting with the IRTF observations. This research has benefited from the SpeX Prism Spectral Libraries, maintained by Adam Burgasser, at http://www.browndwarfs.org/spexprism. This publication makes use of data products from the Two Micron All Sky Survey, which is a joint project of the University of Massachusetts and the Infrared Processing and Analysis Center/California Institute of Technology, funded by the National Aeronautics and Space Administration and the National Science Foundation. This research has benefited from the M, L, and T dwarf compendium housed at DwarfArchives.org and maintained by Chris Gelino, Davy Kirkpatrick, and Adam Burgasser. M.C.L. and E.A.M. were supported by NSF grants AST09-09222 (awarded to M.C.L.) and AST-0709460 (awarded to E.A.M.). E.A.M. was also supported by AFRL Cooperative Agreement FA9451-06-2-0338. This publication makes use of data products from the Wide-field Infrared Survey Explorer, which is a joint project of the University of California, 
Los Angeles, and the Jet Propulsion Laboratory/California Institute of Technology, funded by the National Aeronautics and Space Administration. The United Kingdom Infrared Telescope is operated by the Joint Astronomy Centre on behalf of the Science and Technology Facilities Council of the U.K. This paper makes use of observations processed by the Cambridge Astronomy Survey Unit (CASU) at the Institute of Astronomy, University of Cambridge. The authors thank Mike Irwin and the team at CASU for making the reduced WFCAM data available promptly and Tim Carroll, Thor Wold, Jack Ehle and Watson Varricatt for assisting with UKIRT observations. This research has made use of the SIMBAD database, operated at CDS, Strasbourg, France. The VISTA Data Flow System pipeline processing and science archive are described in Irwin et al. (2004) and Hambly et al. (2008). We have used data from the first data release. This paper makes use of the Topcat software package (Taylor 2005). This research has made use of the Washington Double Star Catalog maintained at the U.S. Naval Observatory. We thank Luca Casagrande, Jackie Faherty, Adam Kraus, Eddie Schlafly, and Josh Schlieder for helpful discussions and our referee Sébastien Lépine for many helpful comments which improved the manuscript. Finally, the authors wish to recognize and acknowledge the very significant cultural role and reverence that the summit of Mauna Kea has always had within the indigenous Hawaiian community. We are most fortunate to have the opportunity to conduct observations from this mountain.

Facilities: IRTF (SpeX), PS1, UKIRT (WFCAM), UH:2.2m (SNIFS)

\section{REFERENCES}

Ahn, C. P., Alexandroff, R., Allende Prieto, C., et al. 2012, ApJS, 203, 21 Aldering, G., Adam, G., Antilogus, P., et al. 2002, Proc. SPIE, 4836, 61 Allen, P. R., Burgasser, A. J., Faherty, J. K., \& Kirkpatrick, J. D. 2012, AJ, 144,62

Aller, K. M., Kraus, A. L., Liu, M. C., et al. 2013, ApJ, 773, 63

Allers, K. N., \& Liu, M. C. 2013, ApJ, 772, 79

Arriagada, P. 2011, ApJ, 734, 70

Artigau, E., Lafrenière, D., Albert, L., \& Doyon, R. 2009, ApJ, 692, 149

Artigau, E., Lafrenière, D., Doyon, R., et al. 2007, ApJL, 659, L49

Bacon, R., Copin, Y., Monnet, G., et al. 2001, MNRAS, 326, 23

Bailey, V., Meshkat, T., Reiter, M., et al. 2014, ApJL, 780, L4

Baraffe, I., Chabrier, G., Allard, F., \& Hauschildt, P. H. 1998, A\&A, 412, 403

Best, W. M. J., Liu, M. C., Magnier, E. A., et al. 2013, ApJ, 777, 84

Bilir, S., Karata, Y., Demircan, O., \& Eker, Z. 2005, MNRAS, 357, 497

Billères, M., Delfosse, X., Beuzit, J.-L., et al. 2005, A\&A, 440, L55

Binks, A. S., \& Jeffries, R. D. 2014, MNRAS, 438, 11

Bochanski, J. J., West, A. A., Hawley, S. L., \& Covey, K. R. 2007, AJ, 133, 531

Bouy, H., Brandner, W., Martn, E. L., et al. 2003, AJ, 126, 1526

Bowler, B. P., Liu, M. C., \& Cushing, M. C. 2009, ApJ, 706, 1114

Bowler, B. P., Liu, M. C., Kraus, A. L., \& Mann, A. W. 2014, ApJ, 784, 65

Bowler, B. P., Liu, M. C., Kraus, A. L., Mann, A. W., \& Ireland, M. J. 2011, ApJ, 743, 148

Bowler, B. P., Liu, M. C., Shkolnik, E. L., et al. 2012, ApJ, 753, 142

Burgasser, A. J. 2007, ApJ, 659, 655

Burgasser, A. J., Cruz, K. L., Cushing, M., et al. 2010, ApJ, 710, 1142

Burgasser, A. J., Geballe, T. R., Leggett, S. K., Kirkpatrick, J. D., \& Golimowski, D. A. 2006, ApJ, 637, 1067

Burgasser, A. J., Kirkpatrick, J. D., Cutri, R. M., et al. 2000, ApJL, 531, L57 Burgasser, A. J., Kirkpatrick, J. D., \& Lowrance, P. J. 2005, AJ, 129, 2849 Burgasser, A. J., McElwain, M. W., Kirkpatrick, J. D., et al. 2004, AJ, 127, 2856 Burningham, B., Cardoso, C. V., Smith, L., et al. 2013, MNRAS, 433, 457 Burningham, B., Pinfield, D. J., Leggett, S. K., et al. 2009, MNRAS, 395, 1237 Burningham, B., Pinfield, D. J., Lucas, P. W., et al. 2010, MNRAS, 406, 1885 Caballero, J. A. 2007, ApJ, 667, 520

Caballero, J. A., \& Montes, D. 2012, Obs, 132, 176

Carpenter, J. M. 2001, AJ, 121, 2851

Casagrande, L., Schönrich, R., Asplund, M., et al. 2011, A\&A, 530, A138 Casali, M., Adamson, A., Oliveira, C. A. D., et al. 2007, A\&A, 467, 777 Chauvin, G., Lagrange, A.-M., Lacombe, F., et al. 2005a, A\&A, 430, 1027
Chauvin, G., Lagrange, A.-M., Zuckerman, B., et al. 2005b, A\&A, 438, L29

Chiu, K., Fan, X., Leggett, S. K., et al. 2006, AJ, 131, 2722

Chubak, C., \& Marcy, G. 2011, BAAS, 43, 434.12

Close, L. M., Siegler, N., Freed, M., \& Biller, B. 2003, ApJ, 587, 407

Covey, K. R., Ivezić, V., Schlegel, D., et al. 2007, AJ, 134, 2398

Cruz, K. L., \& Reid, I. N. 2002, AJ, 123, 34

Cruz, K. L., Reid, I. N., Kirkpatrick, J. D., et al. 2007, AJ, 133, 439

Cruz, K. L., Reid, I. N., Liebert, J., Kirkpatrick, J. D., \& Lowrance, P. J. 2003, AJ, 126,2421

Currie, T., Daemgea, S., Debes, J., et al. 2014, ApJL, 780, L30

Cushing, M. C., Rayner, J. T., \& Vacca, W. D. 2005, ApJ, 623, 1115

Cushing, M. C., Vacca, W. D., \& Rayner, J. T. 2004, PASP, 116, 362

Cutri, R. M., Wright, E. L., Conrow, T., et al. 2012, supplement to the WISE All-Sky Data Release Products (available online at http://wise2.ipac. caltech.edu/docs/release/allsky/expsup/index.html)

Dawson, P. C., \& De Robertis, M. M. 2005, PASP, 117, 1

Day-Jones, A. C., Pinfield, D. J., Napiwotzki, R., et al. 2008, MNRAS, 388,838

Day-Jones, A. C., Pinfield, D. J., Ruiz, M. T., et al. 2011, MNRAS, 410, 705

Deacon, N. R., \& Hambly, N. C. 2007, A\&A, 468, 163

Deacon, N. R., Hambly, N. C., King, R. R., \& McCaughrean, M. J. 2009, MNRAS, 394, 857

Deacon, N. R., Liu, M. C., Magnier, E. A., et al. 2011, AJ, 142, 77

Deacon, N. R., Liu, M. C., Magnier, E. A., et al. 2012a, ApJ, 757, 100

Deacon, N. R., Liu, M. C., Magnier, E. A., et al. 2012b, ApJ, 755, 94

Delgado-Donate, E. J., Clarke, C. J., Bate, M. R., \& Hodgkin, S. T. 2004, MNRAS, 351, 617

Dhital, S., Burgasser, A. J., Looper, D. L., \& Stassun, K. G. 2011, AJ, 141, 7

Dhital, S., West, A. A., Stassun, K. G., \& Bochanski, J. J. 2010, AJ, 139, 2566

Dupuy, T. J., \& Liu, M. C. 2012, ApJS, 201, 19

Dupuy, T. J., Liu, M. C., Bowler, B. P., et al. 2010, ApJ, 721, 1725

Dupuy, T. J., Liu, M. C., \& Ireland, M. J. 2009, ApJ, 692, 729

Emerson, J., \& Sutherland, W. 2010, Msngr, 139, 2

Evans, D. S. 1967, in IAU Symp. 30, Determination of Radial Velocities and their Applications, ed. A. H. Batten \& J. F. Heard (Toronto: Academic Press), 57

Faherty, J. K., Burgasser, A. J., Bochanski, J. J., et al. 2011, AJ, 141, 71

Faherty, J. K., Burgasser, A. J., Cruz, K. L., et al. 2009, AJ, 137, 1

Faherty, J. K., Burgasser, A. J., West, A. A., et al. 2010, AJ, 139, 176

Fujii, M. S., \& Portegies Zwart, S. 2011, Sci, 334, 1380

Gauza, B., Béjar, V. J. S., Rebolo, R., et al. 2012, MNRAS, 427, 2457

Geissler, K., Metchev, S. A., Pham, A., et al. 2012, ApJ, 746, 44

Giclas, H. L., Burnham, R., \& Thomas, N. G. 1967, LowOB, 141, 49

Gizis, J. E., Kirkpatrick, J. D., \& Wilson, J. C. 2001, AJ, 121, 2185

Gizis, J. E., Monet, D. G., Reid, I. N., Kirkpatrick, J. D., \& Burgasser, A. J. 2000, MNRAS, 311, 385

Goldman, B., Marsat, S., Henning, T., Clemens, C., \& Greiner, J. 2010, MNRAS, 405,1140

Golimowski, D. A., Leggett, S. K., Marley, M. S., et al. 2004, AJ, 127, 3516

Gomes, J. I., Pinfield, D. J., Marocco, F., et al. 2013, MNRAS, 431, 2745

Gontcharov, G. A. 2006, AstL, 32, 759

Gould, A., \& Chaname, J. 2004, ApJS, 150, 455

Gray, R. O., Corbally, C. J., Garrison, R. F., et al. 2006, AJ, 132, 161

Hambly, N. C., Collins, R. S., Cross, N. J. G., et al. 2008, MNRAS, 384, 637

Hamuy, M., Walker, A. R., Suntzeff, N. B., et al. 1992, PASP, 104, 533

Hodgkin, S. T., Irwin, M. J., Hewett, P. C., \& Warren, S. J. 2009, MNRAS, 394,675

Hog, E., Fabricius, C., Makarov, V. V., et al. 2000, A\&A, 30, 27

Hovhannisyan, L. R., Mickaelian, A. M., Weedman, D. W., et al. 2009, AJ, 138,251

Howard, A. W., Johnson, J. A., W. Marcy, G., et al. 2010, ApJ, 721, 1467

Hrivnak, B. J., Guinan, E. F., \& Lu, W. 1995, ApJ, 455, 300

Huélamo, N., Nürnberger, D. E. A., Ivanov, V. D., et al. 2010, A\&A, 521, L54 Innes, R. T. A. 1915, CiUO, 30

Ireland, M. J., Kraus, A., Martinache, F., Law, N., \& Hillenbrand, L. A 2011, ApJ, 726, 113

Irwin, M. J., Lewis, J., Hodgkin, S., et al. 2004, Proc. SPIE, 5493, 411 Isaacson, H., \& Fischer, D. 2010, ApJ, 725, 875

Kendall, T., Tamura, M., Tinney, C. G., et al. 2007, A\&A, 466, 1059

Kenyon, S. J., \& Hartmann, L. 1995, ApJS, 101, 117

King, R. R., McCaughrean, M. J., Homeier, D., et al. 2010, A\&A, 510, A99 Kirkpatrick, J. D., Cushing, M. C., Gelino, C. R., et al. 2011, ApJS, 197, 19 Kirkpatrick, J. D., Dahn, C. C., Monet, D. G., et al. 2001, AJ, 121, 3235 Kirkpatrick, J. D., Looper, D. L., Burgasser, A. J., et al. 2010, ApJS, 190, 100 Kirkpatrick, J. D., Reid, I. N., Liebert, J., et al. 1999, ApJ, 519, 802 Kirkpatrick, J. D., Reid, I. N., Liebert, J., et al. 2000, AJ, 120, 447 
Kouwenhoven, M. B. N., Goodwin, S. P., Parker, R. J., et al. 2010, MNRAS, 404, 1835

Kraus, A. L., \& Hillenbrand, L. A. 2007, AJ, 136, 2340

Kraus, A. L., Ireland, M. J., Cieza, L. A., et al. 2014, ApJ, 781, 20

Kuzuhara, M., Tamura, M., Ishii, M., et al. 2011, AJ, 141, 119

Lafrenière, D., Jayawardhana, R., \& van Kerkwijk, M. H. 2008, ApJL, 689, L153

Lafrenière, D., Jayawardhana, R., \& van Kerkwijk, M. H. 2010, ApJ, 719, 497

Lantz, B. 2004, Proc. SPIE, 5249, 146

Latham, D. W. 2004, in Spectroscopically and Spatially Resolving the Components of the Close Binary Stars, Vol. 318, ed. R. W. Hilditch, H. Hensberge, \& K. Pavlovsk (San Francisco, CA: ASP), 276

Latham, D. W., Stefanik, R. P., Torres, G., et al. 2002, AJ, 124, 1144

Law, N. M., Dhital, S., Kraus, A., Stassun, K. G., \& West, A. A. 2010, ApJ, 720,1727

Lawrence, A., Warren, S. J., Almaini, O., et al. 2007, MNRAS, 379, 1599

Lee, Y. S., Beers, T. C., Prieto, C. A., et al. 2011, AJ, 141, 90

Lépine, S. 2005, AJ, 130, 1680

Lépine, S., \& Bongiorno, B. 2007, AJ, 133, 889

Lépine, S., \& Gaidos, E. 2011, AJ, 142, 138

Lépine, S., Hilton, E. J., Mann, A. W., et al. 2013, AJ, 145, 102

Lépine, S., \& Shara, M. M. 2005, AJ, 129, 1483

Limoges, M.-M., Lépine, S., \& Bergeron, P. 2013, AJ, 145, 136

Liu, M. C., Deacon, N. R., Magnier, E. A., et al. 2011, ApJL, 740, L32

Liu, M. C., Dupuy, T. J., \& Ireland, M. J. 2008, ApJ, 689, 436

Liu, M. C., Dupuy, T. J., \& Leggett, S. K. 2010, ApJ, 722, 311

Liu, M. C., Leggett, S. K., \& Chiu, K. 2007, ApJ, 660, 1507

Liu, M. C., Leggett, S. K., Golimowski, D. A., et al. 2006, ApJ, 647, 1393

Loutrel, N. P., Luhman, K. L., Lowrance, P. J., \& Bochanski, J. J. 2011, ApJ, 739,81

Lowrance, P. J., McCarthy, C., Becklin, E. E., et al. 1999, ApJL, 512, L69

Lowrance, P. J., Schneider, G., Kirkpatrick, J. D., et al. 2000, ApJ, 541, 390

Luhman, K. L., Burgasser, A. J., \& Bochanski, J. J. 2011, ApJL, 730, L9

Luhman, K. L., Loutrel, N. P., McCurdy, N. S., et al. 2012, ApJ, 760, 152

Luhman, K. L., Patten, B. M., Marengo, M., et al. 2007, ApJ, 654, 570

Mace, G. N., Kirkpatrick, J. D., Cushing, M. C., et al. 2013, ApJ, 777, 36

Magnier, E. A., Liu, M., Monet, D. G., \& Chambers, K. C. 2008, IAU Symp.

248, A Giant Step: from Milli- to Micro-arcsecond Astrometry (Cambridge: Cambridge Univ. Press), 553

Maldonado, J., Martínez-Arnáiz, R. M., Eiroa, C., Montes, D., \& Montesinos, B. 2010, A\&A, 521, A12

Mamajek, E. E., \& Hillenbrand, L. A. 2008, ApJ, 687, 1264

Mamajek, E. E., Meyer, M. R., \& Liebert, J. 2002, AJ, 124, 1670

Mann, A. W., Brewer, J. M., Gaidos, E., Lépine, S., \& Hilton, E. J. 2013a, AJ, 145,52

Mann, A. W., Deacon, N. R., Gaidos, E., et al. 2014, AJ, 147, 160

Mann, A. W., Gaidos, E., \& Ansdell, M. 2013b, ApJ, 779, 188

Marois, C., Macintosh, B., \& Barman, T. 2007, ApJL, 654, L151

Martín, E. L. 1999, Sci, 283, 1718

Massarotti, A., Latham, D. W., Stefanik, R. P., \& Fogel, J. 2008, AJ, 135, 209

McCaughrean, M. J., Close, L. M., Scholz, R.-D., et al. 2004, A\&A, 413, 1029

Metchev, S. A., \& Hillenbrand, L. A. 2006, ApJ, 651, 1166

Montes, D., López-Santiago, J., Gálvez, M., et al. 2001, MNRAS, 328, 45

Mugrauer, M., Seifahrt, A., \& Neuhauser, R. 2007, MNRAS, 378, 1328

Mugrauer, M., Seifahrt, A., Neuhäuser, R., \& Mazeh, T. 2006, MNRAS, 373, L31

Murray, D. N., Burningham, B., Jones, H. R. A., et al. 2011, MNRAS, 414, 575

Mužić, K., Radigan, J., Jayawardhana, R., et al. 2012, AJ, 144, 180

Naud, M.-E., Artigau, E., Malo, L., et al. 2014, ApJ, 787, 5

Neuhaeuser, R., \& Guenther, E. W. 2004, A\&A, 420, 647

Neuhaeuser, R., Guenther, E. W., Wuchterl, G., et al. 2005, A\&A, 435, L13

Nordstrom, B., Mayor, M., Andersen, J., et al. 2004, A\&A, 418, 989

Pecaut, M. J., \& Mamajek, E. E. 2013, ApJS, 208, 9

Pecaut, M. J., Mamajek, E. E., \& Bubar, E. J. 2012, ApJ, 746, 154
Phan-Bao, N., Bessell, M. S., Martín, E. L., et al. 2008, MNRAS, 383, 831 Pinfield, D. J., Burningham, B., Lodieu, N., et al. 2012, MNRAS, 422, 1922 Pinfield, D. J., Jones, H. R. A., Lucas, P. W., et al. 2006, MNRAS, 368, 1281

Qian, S. B., Soonthornthum, B., Xiang, F. Y., Zhu, L. Y., \& He, J. J. 2004, AN, 325,714

Radigan, J., Lafrenière, D., Jayawardhana, R., \& Doyon, R. 2008, ApJ, 689,471

Radigan, J., Lafrenière, D., Jayawardhana, R., \& Doyon, R. 2009, ApJ, 698, 405

Raghavan, D., McAlister, H. A., Henry, T. J., et al. 2010, ApJS, 190, 1

Ramírez, I., Meléndez, J., \& Asplund, M. 2009, A\&A, 508, L17

Rayner, J. T., Cushing, M. C., \& Vacca, W. D. 2009, ApJS, 185, 289

Rayner, J. T., Toomey, D. W., Onaka, P. M., et al. 2003, PASP, 115, 362

Rebolo, R. 1998, Sci, 282, 1309

Reid, I. N., Cruz, K. L., Kirkpatrick, J. D., et al. 2008, AJ, 136, 1290

Reid, I. N., Gizis, J. E., Kirkpatrick, J. D., \& Koerner, D. W. 2001, AJ, 121, 489

Reid, I. N., Hawley, S. L., \& Gizis, J. E. 1995, AJ, 110, 1838

Reid, I. N., \& Walkowicz, L. M. 2006, PASP, 118, 671

Reipurth, B., \& Mikkola, S. 2012, Natur, 492, 221

Reylé, C., Delorme, P., Artigau, E., et al. 2013, A\&A, 561, A66, 8

Robinson, S. E., Ammons, S. M., Kretke, K. A., et al. 2007, ApJS, 169, 430

Rojas-Ayala, B., Covey, K. R., Muirhead, P. S., \& Lloyd, J. P. 2010, ApJL, 720, L113

Rucinski, S. M., Pribulla, T., \& van Kerkwijk, M. H. 2007, AJ, 134, 2353

Salim, S., \& Gould, A. 2003, ApJ, 582, 1011

Saumon, D., Marley, M. S., Leggett, S. K., et al. 2007, ApJ, 656, 1136

Schmitt, J. H. M. M., Fleming, T. A., \& Giampapa, M. S. 1995, ApJ, 450, 392

Scholz, R.-D. 2010a, A\&A, 515, A92

Scholz, R.-D. 2010b, A\&A, 510, L8

Scholz, R.-D., Lodieu, N., Ibata, R., et al. 2004, MNRAS, 347, 685

Scholz, R.-D., McCaughrean, M. J., Lodieu, N., \& Kuhlbrodt, B. 2003, A\&A, 398, L29

Seifahrt, A., Guenther, E., \& Neuhäuser, R. 2005, A\&A, 440, 967

Shkolnik, E., Liu, M. C., \& Reid, I. N. 2009, ApJ, 699, 649

Skrutskie, M. F., Cutri, R. M., Stiening, R., et al. 2006, AJ, 131, 1163

Smith, L., Lucas, P. W., Burningham, B., et al. 2014, MNRAS, 437, 3603

Song, I., Zuckerman, B., \& Bessell, M. S. 2003, ApJ, 599, 342

Taylor, M. 2005, in ASP Conf. Ser. 347, Astronomical Data Analysis Software and Systems XIV, ed. P. Shopbell, M. Britton, \& R. Ebert (San Francisco, CA: ASP), 29

Tetzlaff, N., Neuhäuser, R., \& Hohle, M. M. 2011, MNRAS, 410, 190

Tokovinin, A., \& Lépine, S. 2012, AJ, 144, 102

Tonry, J. L., Stubbs, C. W., Lykke, K. R., et al. 2012, ApJ, 750, 99

Umbreit, S., Burkert, A., Henning, T., Mikkola, S., \& Spurzem, R. 2005, ApJ, 623,940

Vacca, W. D., Cushing, M. C., \& Rayner, J. T. 2003, PASP, 115, 389

Valenti, J. A., \& Fischer, D. A. 2005, ApJS, 159, 141

van Biesbroeck, G. 1944, AJ, 51, 61

van Dam, M. A., Bouchez, A. H., Le Mignant, D., et al. 2006, PASP, 118, 310 van Leeuwen, F. 2007, A\&A, 474, 653

Voges, W., Aschenbach, B., Boller, T., et al. 2000, IAUC, 7432, 3

Webb, R. A., Zuckerman, B., Platais, I., et al. 1999, ApJL, 512, L63

Weinberg, M. D., Shapiro, S. L., \& Wasserman, I. 1987, ApJ, 312, 367

West, A. A., Hawley, S. L., Bochanski, J. J., et al. 2008, AJ, 135, 785

White, R. J., Gabor, J. M., \& Hillenbrand, L. A. 2007, AJ, 133, 2524

White, R. J., Ghez, A. M., Reid, I. N., \& Schultz, G. 1999, ApJ, 520, 811

Wielen, R., Schwan, H., Dettbarn, C., et al. 2000, Sixth Catalogue of Fundamental Stars (Karlsruhe: Verlag G. Braun)

Wilson, J. C., Kirkpatrick, J. D., Gizis, J. E., et al. 2001, AJ, 122, 1989

Wizinowich, P. L., Le Mignant, D., Bouchez, A. H., et al. 2006, PASP, 118, 297

Wright, E. L., Eisenhardt, P. R. M., Mainzer, A. K., et al. 2010, AJ, 140, 1868

Wright, E. L., Skrutskie, M. F., Kirkpatrick, J. D., et al. 2013, AJ, 145, 84

Yelda, S., Lu, J. R., Ghez, A. M., et al. 2010, ApJ, 725, 331

Zhang, Z. H., Pinfield, D. J., Day-Jones, A. C., et al. 2010, MNRAS, 1834, 1817 